\title{
Post-Model-Selection Inference in Linear Regression Models: An Integrated Review Supplementary Material
}

\author{
Dongliang Zhang * \\ Department of Biostatistics, Johns Hopkins University, Baltimore, MD, USA \\ e-mail: dzhang69@jhu.edu \\ Abbas Khalili \\ Department of Mathematics and Statistics, McGill University, Montréal, QC, Canada \\ e-mail: abbas.khalili@mcgill.ca \\ Masoud Asgharian \\ Department of Mathematics and Statistics, McGill University, Montréal, QC, Canada \\ e-mail: masoud.asgharian2@mcgill.ca
}

\section{Contents}

S1 Motivating Example: Settings and Additional Plots . . . . . . . . . . 3

S2 Sampling Distributions of Post-Model-Selection Parameter Estimators

in Linear Regression Models . . . . . . . . . . . . . . . . . . . . . . . 14

S3 Properties of Coverage Probabilities of Naïve Confidence Intervals for

Population-based Targets in Linear Regression Models . . . . . . . . . 21

S4 Exact Post-Selection Inference (EPoSI): Supplementary Formula . . . 23

S5 Additional Simulation Results . . . . . . . . . . . . . . . . . . 24

S5.1 Model I $(n=40, p=14) \ldots \ldots \ldots \ldots \ldots \ldots$

S5.2 Model II $(n=40, p=14) \ldots \ldots \ldots \ldots \ldots$

${ }^{*}$ Corresponding author 
S5.3 Model III $(n=40, p=14) \ldots \ldots \ldots \ldots \ldots$

S5.4 Model IV $(n=40, p=14) \ldots \ldots \ldots \ldots \ldots$

S5.5 Model I $(n=60, p=18) \ldots \ldots \ldots \ldots \ldots \ldots$

S5.6 Model II $(n=60, p=18) \ldots \ldots \ldots \ldots$. . . . . . . 43

S5.7 Model III $(n=60, p=18) \ldots \ldots \ldots \ldots \ldots$

S5.8 Model IV $(n=60, p=18) \ldots \ldots \ldots \ldots \ldots \ldots$ 


\section{S1. Motivating Example: Settings and Additional Plots}

In this section, we provide the settings of and the remaining plots for our motivating example in Section 3 of the main manuscript. The settings of our motivating example are as follows:

1. Design matrix generation: The design matrix $\boldsymbol{X}$ has $n=50$ independent observations and $p=200$ covariates. Each observation is generated from a multivariate normal random vector with mean zero and equicorrelated variance-covariance structure with pairwise correlation coefficient $\rho=0.9$;

2. True data generating model and response generation: According to the linear model (1) of the main manuscript, the true data generating model is $\boldsymbol{M}_{\text {true }}=\{1,3,4,5,7\}$ and the non-zero population-based regression coefficients are $\beta_{1}^{0}=\beta_{3}^{0}=\beta_{4}^{0}=\beta_{5}^{0}=\beta_{7}^{0}=1.5$. Moreover, 5000 copies of response $\boldsymbol{Y}$ are randomly sampled based on $\boldsymbol{M}_{\text {true }}$ with error $\sigma^{2}=2$;

3. Model selection and regression coefficient estimation: For each sample, we apply LASSO (Tibshirani 1996), elastic net (Zou \& Hastie 2005) and SCAD (Fan \& Li 2001) for model selection. We then refit a linear regression model to the selected covariates and obtain the ordinary least-squares (OLS) estimators;

4. $\mathrm{R}$ implementation: The R package glmnet (Friedman et al. 2010) is used to fit LASSO and elastic net penalized regression models. The package ncvreg (Breheny \& Huang 2011) is used to fit a SCAD penalized regression model. For LASSO and SCAD, 10-fold cross-validation (CV) is used to select the tuning parameter $\lambda$. For SCAD, the other tuning parameter $a$ is set to be 3.7 by the recommendations from Fan \& Li (2001) to minimize the so-called Bayes risk. For elastic net, we conduct two-dimensional 10fold CV search for the optimal pair of tuning parameters;

5. Nominal coverage probability: The nominal coverage probability is $1-\alpha=0.95$.

Next, for $j=\{1,3,4,7\} \subseteq \boldsymbol{M}_{\text {true }}$, we present plots of empirical densities and boxplots of 
(a) $\widehat{\beta}_{j \cdot \widehat{M}}^{\text {Method against }} \widehat{\beta}_{j \cdot M_{\text {true }}}$ (Figures $\left.1-4\right)$;

(b) $T_{j \cdot \overline{\boldsymbol{M}}}^{\text {Method }}$ and $T_{j \cdot \widehat{\boldsymbol{M}}, 0}^{\text {Method }}$ against their true counterparts, $T_{j \cdot \boldsymbol{M}_{\text {true }}}$ and $T_{j \cdot \boldsymbol{M}_{\text {true }}, 0}$ (Figures 5-12).

These plots supplement Figures 1-3 in the main manuscript which contain the results for $j=5 \in \boldsymbol{M}_{\text {true }}$.
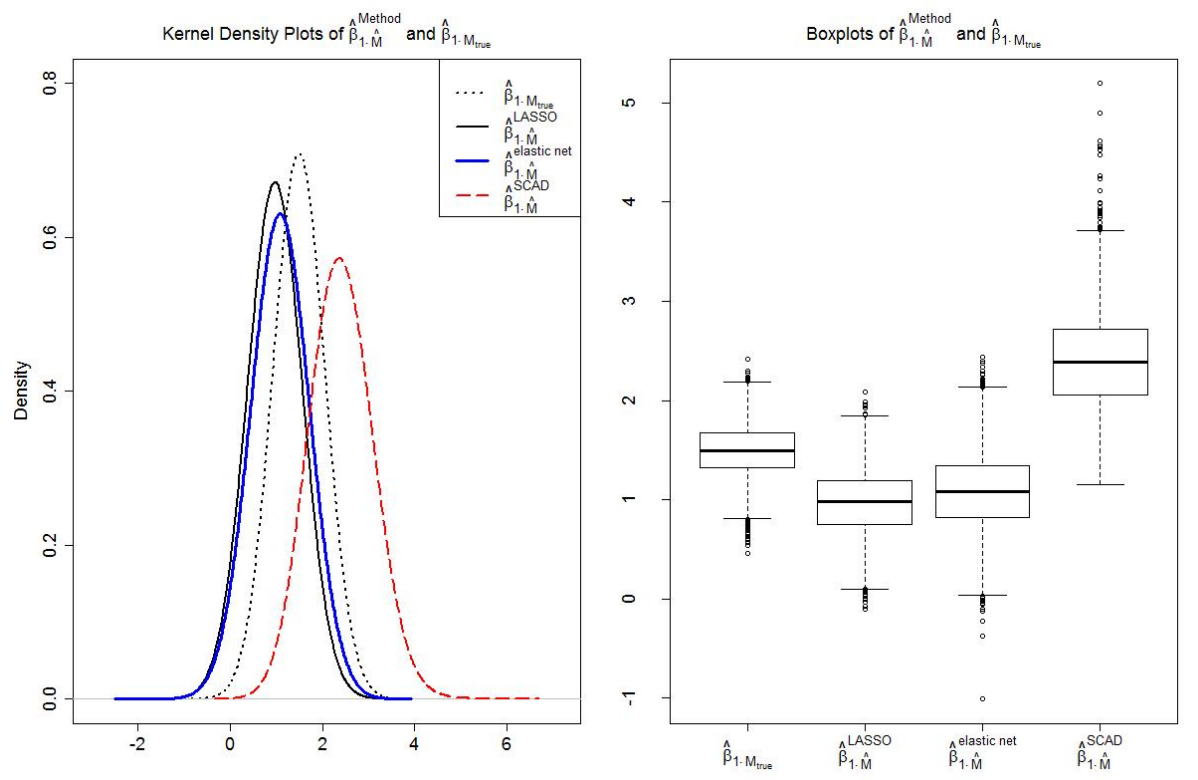

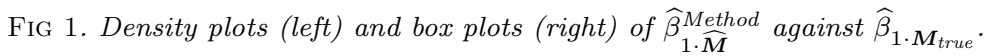



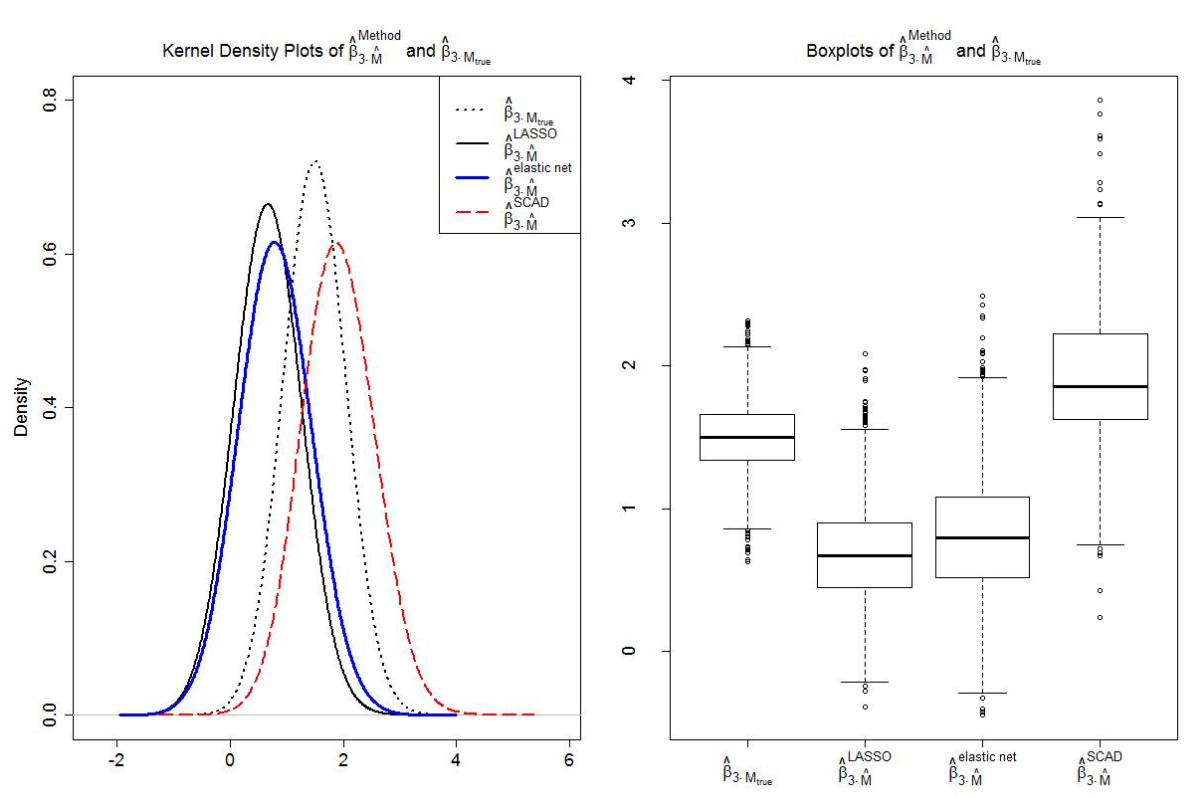

FIG 2. Density plots (left) and box plots (right) of $\widehat{\beta}_{3 \cdot \widehat{M}}^{M e t h o d}$ against $\widehat{\beta}_{3 \cdot M_{\text {true }}}$.

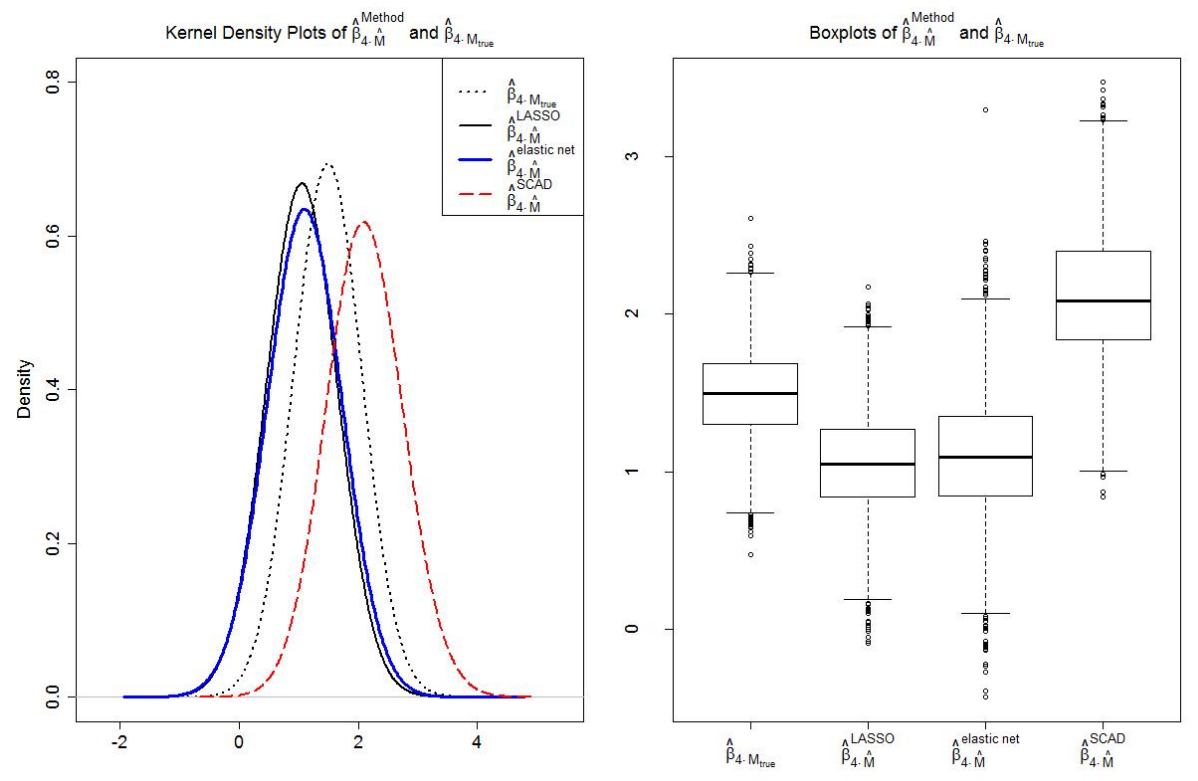

FIG 3. Density plots (left) and box plots (right) of $\widehat{\beta}_{4 \cdot \widehat{M}}^{\text {Method }}$ against $\widehat{\beta}_{4 \cdot M_{\text {true }}}$. 


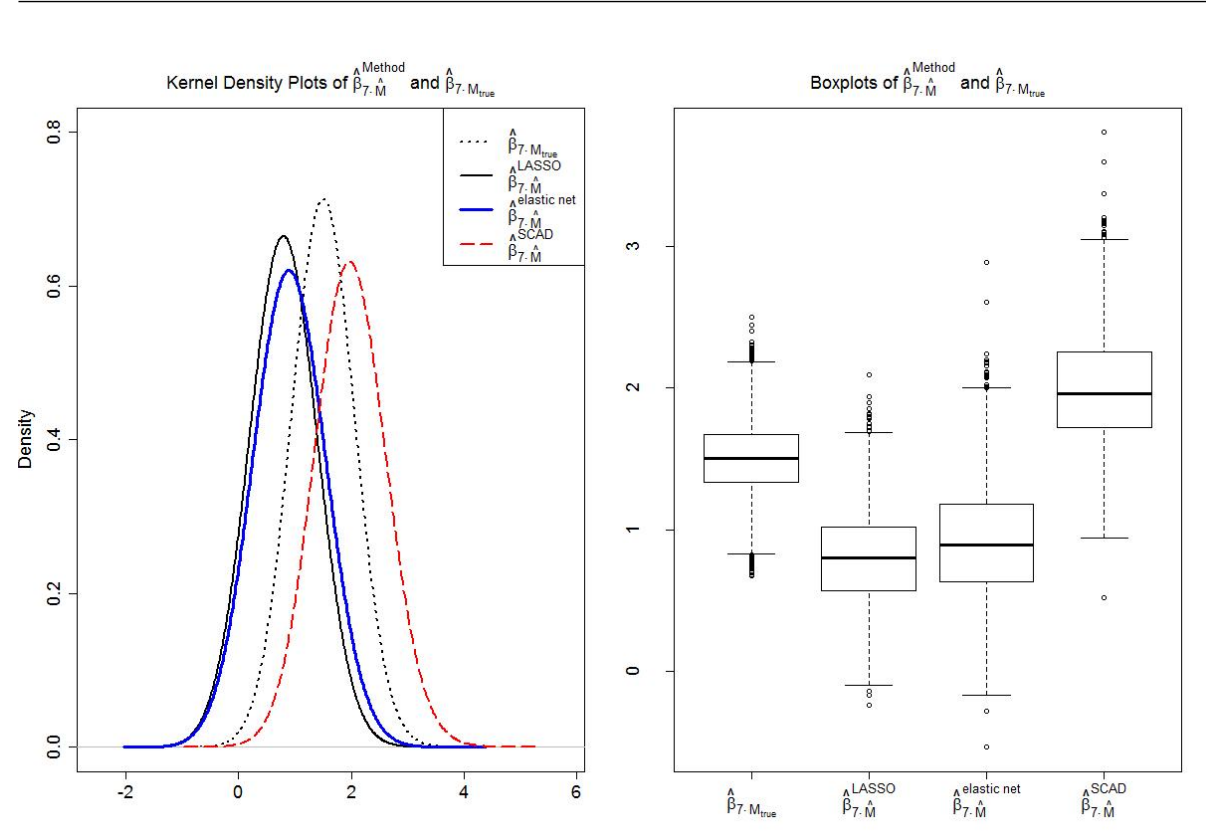

FIG 4. Density plots (left) and box plots (right) of $\widehat{\beta}_{7 \cdot \widehat{M}}^{\text {Method }}$ against $\widehat{\beta}_{7 \cdot M_{\text {true }}}$. 

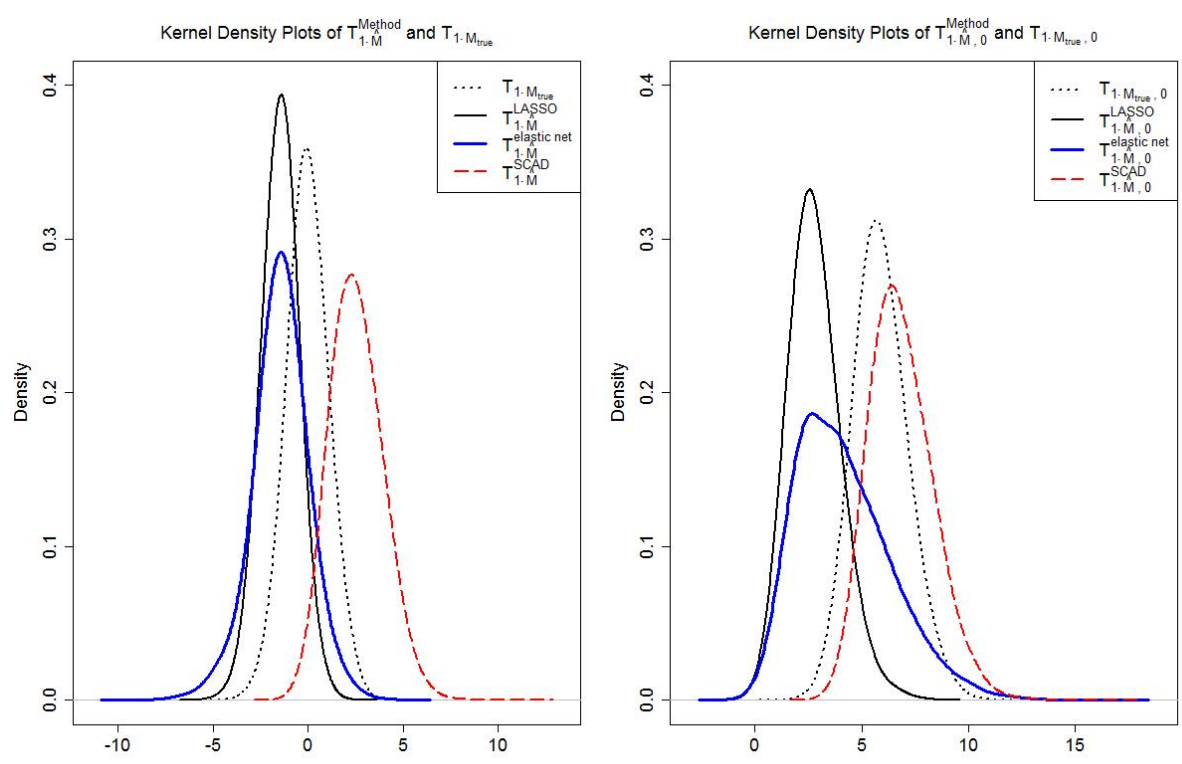

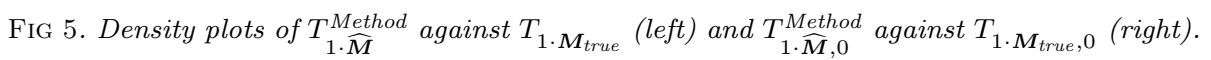



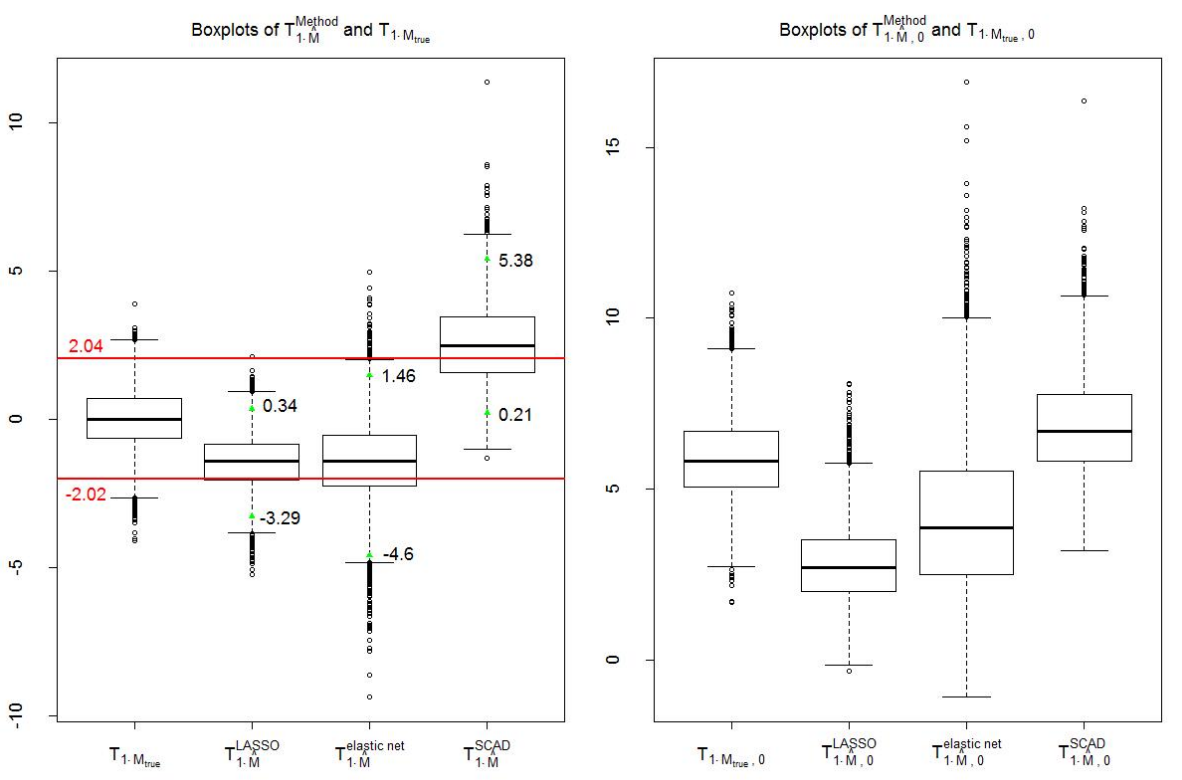

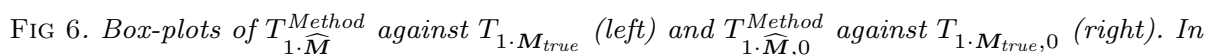
the left panel, the $2.5^{\text {th }}$ and the $97.5^{\text {th }}$ percentiles of each boxplot are indicated. 

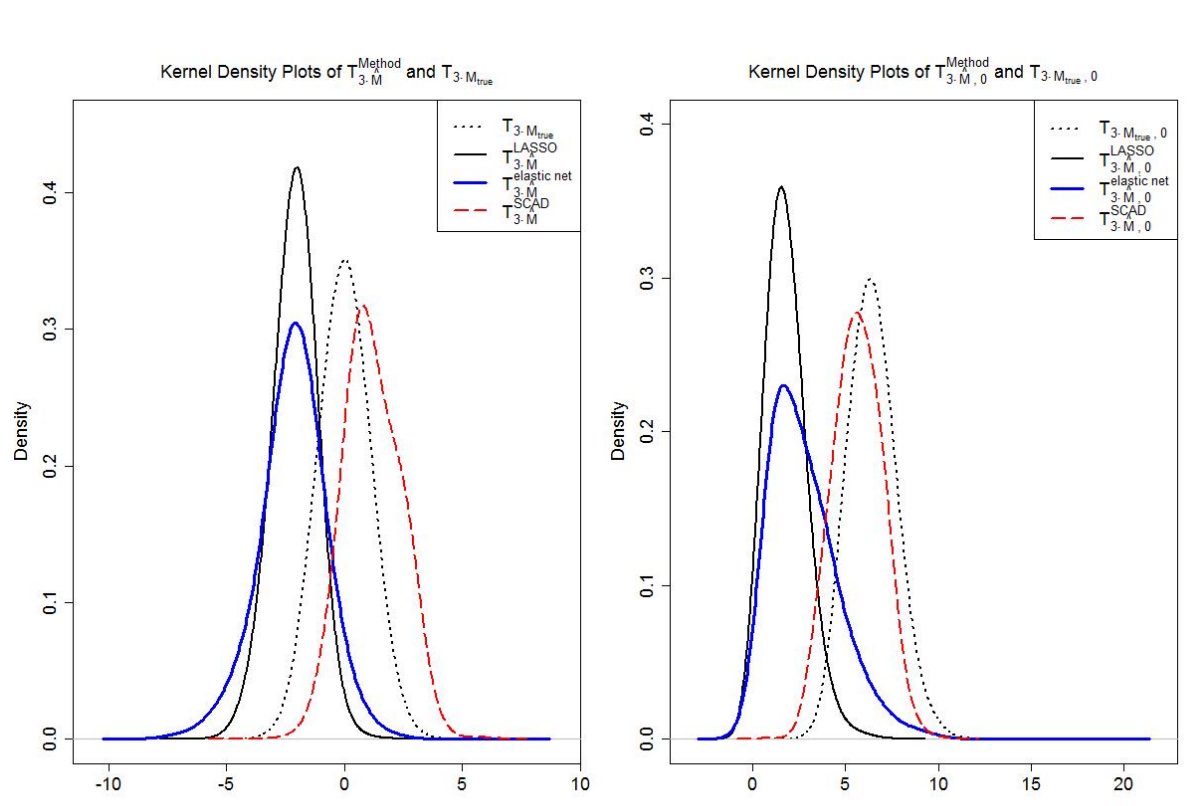

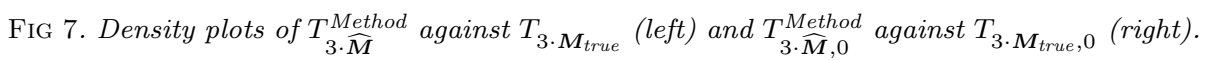



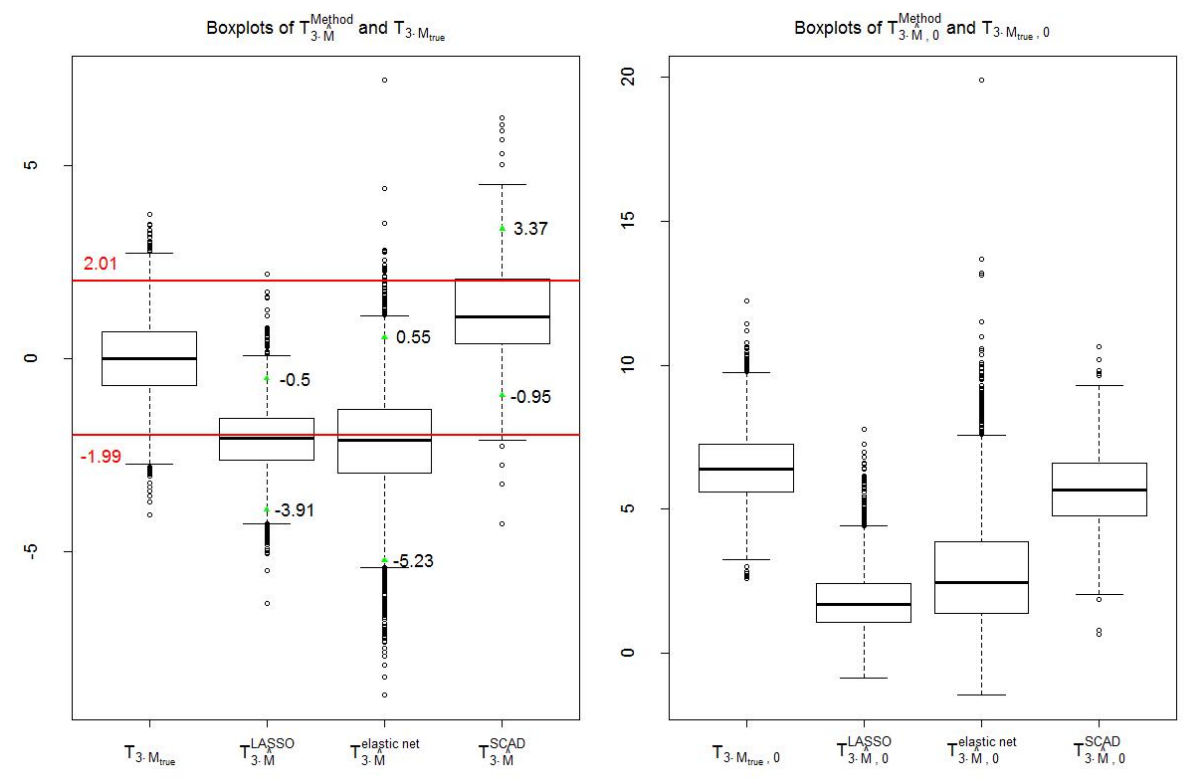

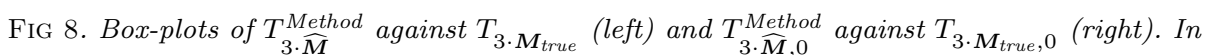
the left panel, the $2.5^{\text {th }}$ and the $97.5^{\text {th }}$ percentiles of each boxplot are indicated. 

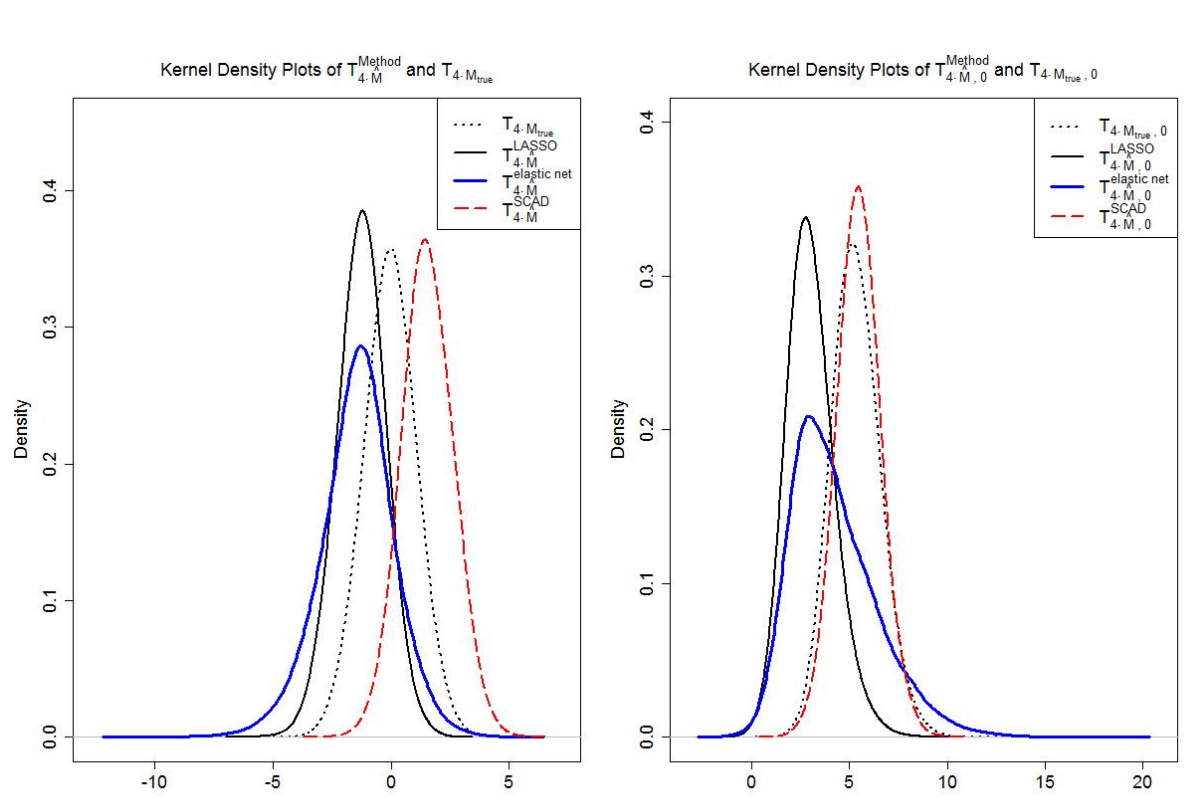

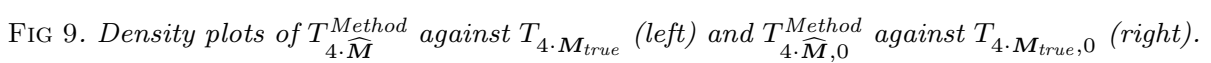



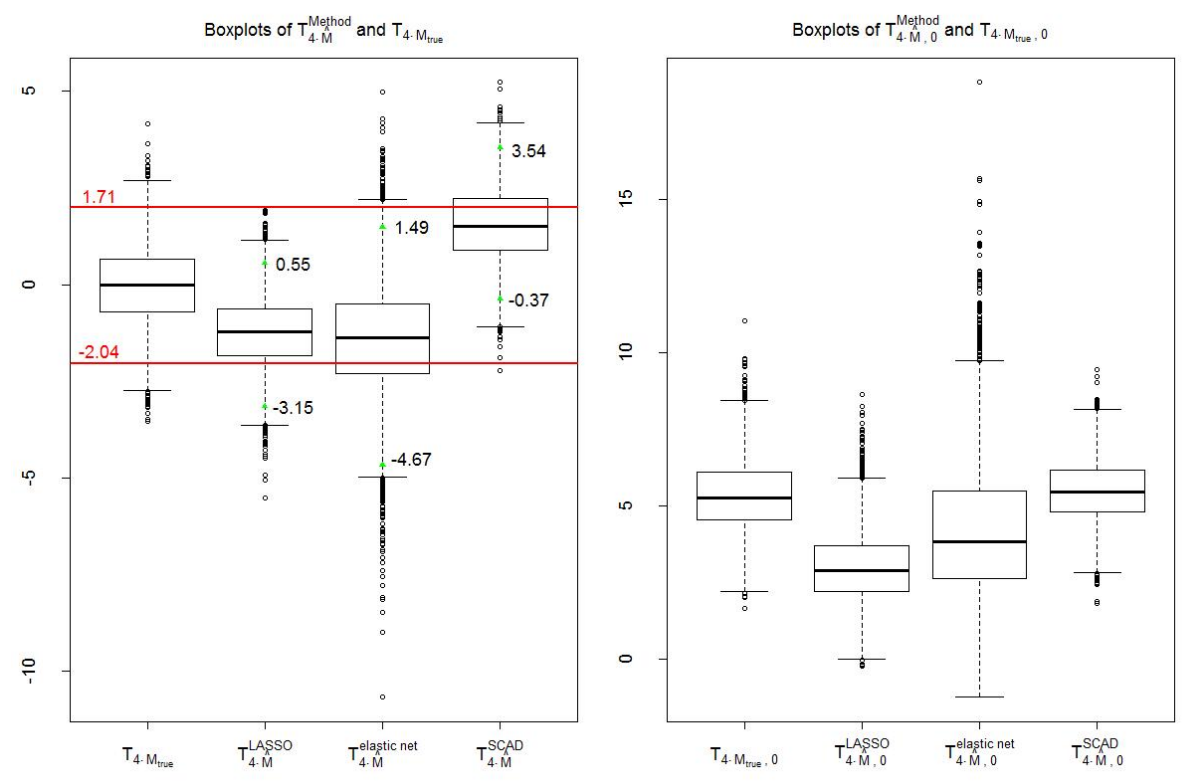

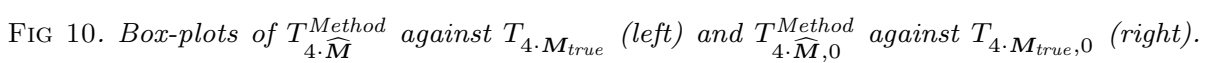
In the left panel, the $2.5^{\text {th }}$ and the $97.5^{\text {th }}$ percentiles of each boxplot are indicated. 

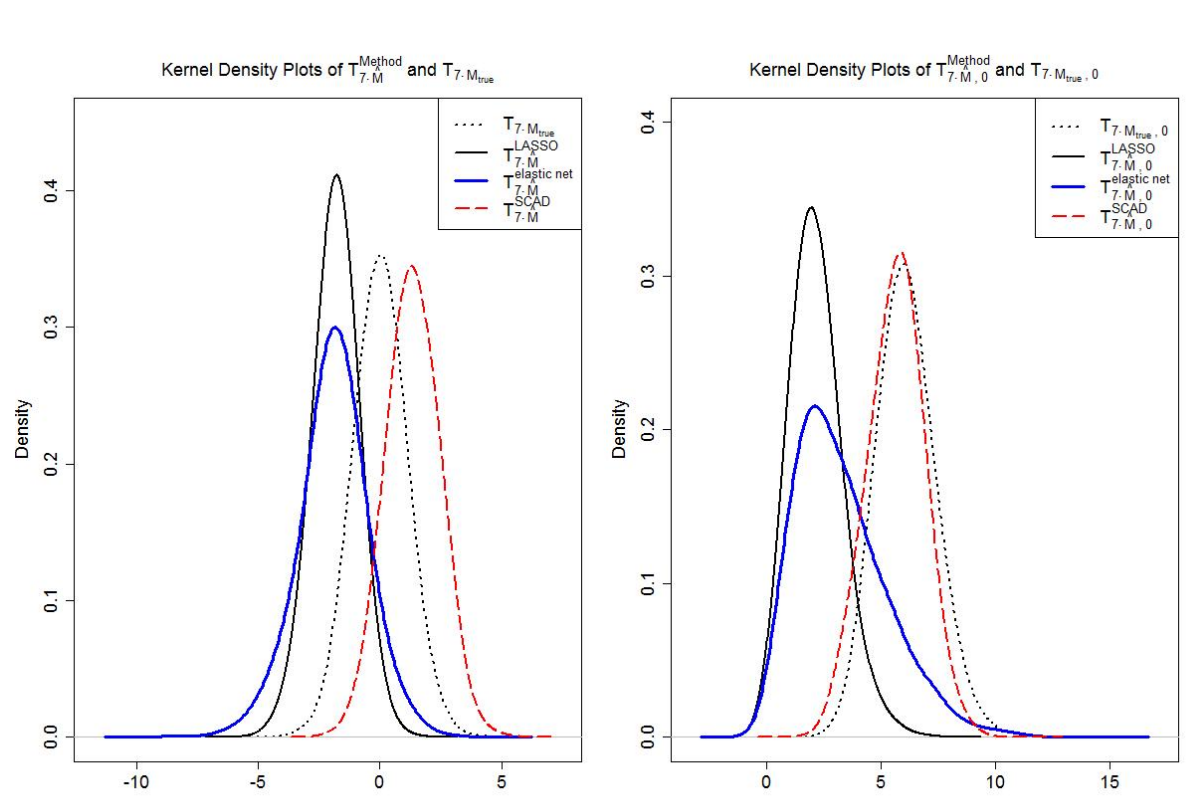

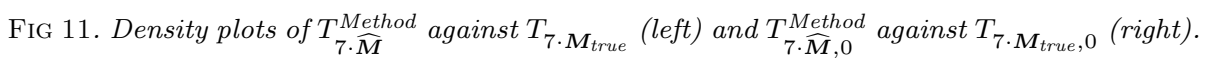



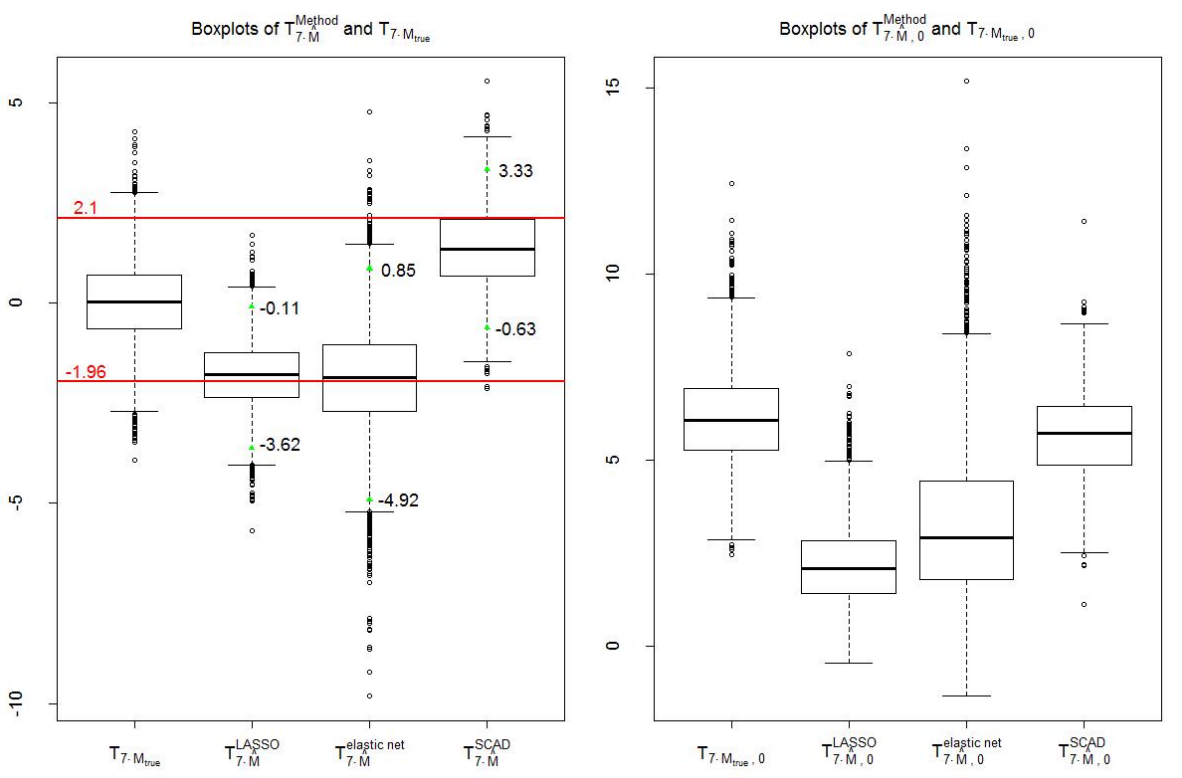

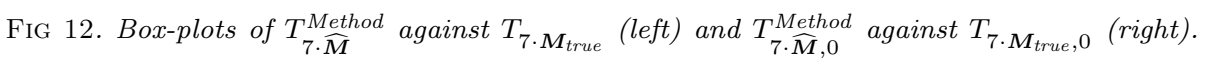
In the left panel, the $2.5^{\text {th }}$ and the $97.5^{\text {th }}$ percentiles of each boxplot are indicated.

\section{S2. Sampling Distributions of Post-Model-Selection Parameter Estimators in Linear Regression Models}

In this section, we provide a detailed review of results pertaining to finite- and

large-sample distributional properties of post-selection parameter estimators in linear regression models. This section substantiates the expositions in Section 4 of the main manuscript.

To the best of our knowledge, despite the fact that problems in relevance to the post-selection inference have been recognized long ago by the 1975 Nobel Memorial Prize laureate in Economic Sciences, Koopmans (1949), systematic and rigorous study of finite- and large-sample distributional properties of postselection parameter estimators in linear regression models did not commence until early nineteen nineties. Pioneered by Benedikt M. Pötscher and Hannes Leeb, such research is focused on the so-called post-model-selection (PMS) estimator (to be defined later). Denoted by $\widetilde{\boldsymbol{\beta}}_{\mathrm{PMS}}$, the PMS estimator has been 
gradually developed by Pötscher (1991), Pötscher \& NovÁk (1998) and Leeb \& Pötscher $(2003,2005,2006,2008)$. It is imperative to note that unlike other commonly-used, albeit non-specific terms, such as "a parameter estimator obtained after model selection", the PMS estimator $\widetilde{\boldsymbol{\beta}}_{\text {PMS }}$ has an explicit and well-defined structure, which is formulated as follows.

Define a submodel $\boldsymbol{M}_{[K]}=\{1,2, \ldots, K\} \subseteq\{1, \ldots, p\}$ where $K$ is known as the order of this submodel such that $1 \leq K \leq p$. Moreover, we assume that the true linear data generating submodel $\boldsymbol{M}_{\text {true }}=\boldsymbol{M}_{\left[\boldsymbol{K}_{\text {true }}\right]}$ for some $K_{\text {true }}$ such that $1 \leq K_{\text {true }} \leq p$, where $K_{\text {true }}$ is known as the minimal true model order for that true model. Let the OLS estimator corresponding to $\boldsymbol{M}_{[K]}$ be $\widehat{\boldsymbol{\beta}}_{\boldsymbol{M}_{[K]}}$. Upon

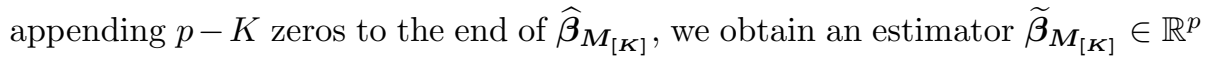
corresponding to the restriction of using the first $K$ covariates for regression coefficient estimation. Considering all possible selected submodels indexed by $\boldsymbol{M}_{[K]}$, the PMS estimator $\widetilde{\boldsymbol{\beta}}_{\mathrm{PMS}}$ is then defined by

$$
\widetilde{\boldsymbol{\beta}}_{\mathrm{PMS}}=\sum_{K=1}^{p} \widetilde{\boldsymbol{\beta}}_{\boldsymbol{M}_{[K]}} \mathbb{1}\left(\widehat{\boldsymbol{M}}=\boldsymbol{M}_{[K]}\right) .
$$

Considering both consistent and inconsistent model selectors, Pötscher (1991) examines asymptotic theories for both conditional and unconditional distributions of $\widetilde{\boldsymbol{\beta}}_{\text {PMS }}$. He first shows [ibid., Lemmas 1 and 2, Section 2] that using consistent model selectors, the asymptotic distribution of $\widetilde{\boldsymbol{\beta}}_{\text {PMS }}$ is unaffected by the data-driven model selection, since

$$
\lim _{n \rightarrow \infty} \mathbb{P}\left(\widehat{\boldsymbol{M}}=\boldsymbol{M}_{\text {true }}\right)=1 \Rightarrow \lim _{n \rightarrow \infty} \mathbb{P}\left(f(\widehat{\boldsymbol{M}})=f\left(\boldsymbol{M}_{\text {true }}\right)=1,\right.
$$

where $f(\widehat{\boldsymbol{M}})$ denotes any statistic based on a selected submodel $\widehat{\boldsymbol{M}}$. In particular, equation (2) implies that the finite-sample regression coefficient estimators and any test statistics obtained from the selected submodel converge in probability to their asymptotic counterparts obtained by fitting a linear regression model to the true submodel. As such, for a fixed true regression coefficient $\boldsymbol{\beta}^{0}$, the distribution of $\sqrt{n}\left(\widetilde{\boldsymbol{\beta}}_{\mathrm{PMS}}-\boldsymbol{\beta}^{0}\right)$ is asymptotically Gaussian.

In contrast, when using inconsistent model selectors such as a sequential hypothesis testing procedure [ibid., Section 3.2], Pötscher explicitly derives [ibid., Theorem 1] the asymptotic unconditional distribution of $\sqrt{n}\left(\widetilde{\boldsymbol{\beta}}_{\mathrm{PMS}}-\boldsymbol{\beta}^{0}\right)$, which 
is given by

$$
\lim _{n \rightarrow \infty} \mathbb{P}\left(\sqrt{n}\left(\widetilde{\boldsymbol{\beta}}_{\mathrm{PMS}}-\boldsymbol{\beta}^{0}\right) \leq \boldsymbol{x}\right)=\sum_{K=K_{\text {true }}}^{p} \gamma_{K} F_{K}(\boldsymbol{x}), \boldsymbol{x} \in \mathbb{R}^{p},
$$

where $\gamma_{K}$ is the limiting selection probability of submodel $\boldsymbol{M}_{[K]}, F_{K}(\cdot)$ has a complicated structure and is a normal cumulative distribution function (CDF) if and only if $K=K_{\text {true }}$. Moreover, for $K \geq K_{\text {true }}$, Pötscher shows [ibid., Theorem $1]$ that the large-sample conditional distribution of $\sqrt{n}\left(\widetilde{\boldsymbol{\beta}}_{\mathrm{PMS}}-\boldsymbol{\beta}^{0}\right)$ is given by

$$
\lim _{n \rightarrow \infty} \mathbb{P}\left(\sqrt{n}\left(\widetilde{\boldsymbol{\beta}}_{\mathrm{PMS}}-\boldsymbol{\beta}^{0}\right) \leq \boldsymbol{x} \mid \widehat{\boldsymbol{M}}=\boldsymbol{M}_{[K]}\right)=F_{K}(\boldsymbol{x}) .
$$

From (3), we observe that conditioning on the over-estimation of the minimal true model order $K_{\text {true }}$, which is denoted by $\boldsymbol{M}_{\left[\boldsymbol{K}_{\text {true] }}\right.} \subsetneq \widehat{\boldsymbol{M}}=\boldsymbol{M}_{[K]}$ for some $K>K_{\text {true }}$, the conditional distribution function given in (3) is complicated and difficult to interpret. Therefore, in this case, there exists discrepancy between the asymptotic conditional distribution of the PMS estimator and asymptotic normality of OLS estimator obtained from fitting a fixed model $\boldsymbol{M}_{[\boldsymbol{K}]}$. On the other hand, when correctly estimating the minimal true model order $K_{\text {true }}$, which is denoted by $\widehat{\boldsymbol{M}}=\boldsymbol{M}_{[\boldsymbol{K}]}=\boldsymbol{M}_{\left[\boldsymbol{K}_{\text {true }}\right.}$ for $K=K_{\text {true }}$, the asymptotic conditional distribution of the PMS estimator $\widetilde{\boldsymbol{\beta}}_{\text {PMS }}$ coincides with asymptotic normality of the OLS estimator. In other words, data-driven model selection has no impact on asymptotic distribution of the PMS estimator $\widetilde{\boldsymbol{\beta}}_{\mathrm{PMS}}$ if and only if we select the correct model order.

There are three limitations of the results in Pötscher (1991):

(I) Under inconsistent model selectors, the asymptotic conditional distribution in (3) is derived for conditioning on both correct and over-estimation of minimal true model order $\boldsymbol{M}_{\left[\boldsymbol{K}_{\text {true }}\right.} \subseteq \widehat{\boldsymbol{M}}=\boldsymbol{M}_{[\boldsymbol{K}]}$ where $K \geq K_{\text {true }}$. Analogous formula is not given when conditioning on the under-estimation of minimal true model order, denoted by $\widehat{\boldsymbol{M}}=\boldsymbol{M}_{[K]} \subsetneq \boldsymbol{M}_{\left[K_{\text {true }}\right.}$ for some $K<K_{\text {true }}$;

(II) Under inconsistent model selectors, the pointwise conditional convergence result in (3) assumes the true regression coefficient $\boldsymbol{\beta}^{\mathbf{0}}$ is fixed. This does not provide insights into the quality of the asymptotic approximation. In other words, we have no information regarding the uniformity in the large-sample approximation with respect to $\boldsymbol{\beta}^{\mathbf{0}}$; 
(III) Under consistent model selectors, the uniformity of the large-sample approximation is unknown either.

The first issue (I) pertaining to the absence of large-sample distribution of the PMS estimator $\widetilde{\boldsymbol{\beta}}_{\mathrm{PMS}}$ conditioning on under-estimation of minimal true model order is fully addressed in Leeb \& Pötscher (2003). In addition, Leeb \& Pötscher (2003) have also derived [ibid., Section 3] both conditional (on over- and underestimation of minimal true model order $\left.K_{\text {[true] }}\right)$ and unconditional finite-sample distributions of the PMS estimator $\widetilde{\boldsymbol{\beta}}_{\text {PMS }}$ in both cases of known and unknown errors $\sigma^{2}$. Their formulations are intractable and difficult to interpret.

The second issue (II) with respect to the uniformity of conditional largesample approximation under inconsistent model selectors is addressed by Pötscher \& NovÁk (1998) through intensive Monte Carlo simulations. In distributed lag models, the authors consider two inconsistent model selectors, which are minimizing AIC and the aforementioned sequential hypothesis testing procedure. Upon plotting and comparing empirical finite-sample and asymptotic conditional densities, they have acquired the following critical observations:

(i) When the true signals $\boldsymbol{\beta}^{0}$ are not very strong, simulations indicate (Figures 1.2 and 1.3, and 2.4 to 2.6 from Pötscher \& NovÁk (1998)) that the finitesample conditional distribution of $\sqrt{n}\left(\widetilde{\boldsymbol{\beta}}_{\mathrm{PMS}}-\boldsymbol{\beta}^{0}\right)$ is poorly predicted by its asymptotic counterpart. The authors reason that although the probability of under-estimating the minimal true model order is asymptotically zero, this probability plays a crucial role in finite-sample cases. This leads to non-uniformity in the convergence of the finite-sample conditional distribution of the PMS estimator to its asymptotic equivalent near lowerdimensional models. However, for some other values of $\boldsymbol{\beta}^{0}$, the behavior of finite-sample densities of the PMS estimator is well predicted by its asymptotic counterparts, further highlighting the non-uniformity in convergence.

(ii) In contrast, when conditioning on over-estimation of the minimal true model order, simulations show that there is consistent satisfactory agreement between finite-sample distribution of $\sqrt{n}\left(\widetilde{\boldsymbol{\beta}}_{\mathrm{PMS}}-\boldsymbol{\beta}^{0}\right)$ and its asymptotic approximation, even for a small sample size such as $n=50$. 
Apart from the above two observations, the authors have also confirmed that conditioning on over-estimation of minimal true order, there exists dissimilarities between the finite- and large-sample densities of the PMS estimators, and the OLS estimator obtained when fixing the corresponding model order. In such cases, they find that empirical densities of the PMS estimators are more spreadout than the OLS estimators. Furthermore, bimodality is observed in the density plots of the PMS estimators. This discrepancy serves as numerical corroboration of the corresponding theories in Leeb \& Pötscher (2003).

The third issue (III) with respect to the limiting behavior of the the PMS estimator under consistent (and conservative) model selection framework is given an illustrative treatment by Leeb \& Pötscher (2005). The authors consider a simple design matrix only containing two covariates, $\boldsymbol{x}_{\mathbf{1}}$ and $\boldsymbol{x}_{\mathbf{2}}$, where the first covariate $\boldsymbol{x}_{1}$ is always retained in a selected submodel. The full linear regression model is then given by

$$
\boldsymbol{Y}=\beta_{1}^{0} \boldsymbol{x}_{\mathbf{1}}+\beta_{2}^{0} \boldsymbol{x}_{\mathbf{2}}+\boldsymbol{\epsilon}, \boldsymbol{\epsilon} \sim \mathcal{N}\left(\mathbf{0}, \sigma^{2} \boldsymbol{I}\right)
$$

so the parameter of interest is $\beta_{1}^{0}$, and $\beta_{2}^{0}$ is a nuisance parameter. By $(1)$, the PMS estimator of $\beta_{1}^{0}$, denoted by $\widetilde{\beta}_{1, \mathrm{PMS}}$, is formulated as

$$
\widetilde{\beta}_{1, \mathrm{PMS}}=\widehat{\beta}_{1 \cdot \mathbf{R}} \mathbb{1}(\widehat{M}=\mathbf{R})+\widehat{\beta}_{1 \cdot \mathbf{U}} \mathbb{1}(\widehat{M}=\mathbf{U})
$$

where $\mathbf{R}$ denotes the restricted submodel containing only $\boldsymbol{x}_{\mathbf{1}}, \mathbf{U}$ denotes the unrestricted full model containing both covariates, and $\widehat{\beta}_{1 \cdot \mathbf{R}}$ and $\widehat{\beta}_{1} \cdot \mathbf{U}$ respectively denote the OLS estimator for the parameter of interest based on models $\mathbf{R}$ and U. Using minimum BIC as the model selector, the authors explicitly construct the finite-sample density of $\sqrt{n}\left(\widetilde{\beta}_{1, \mathrm{PMS}}-\beta_{1}^{0}\right)$ as

$$
\begin{aligned}
f_{n, \beta_{1}^{0}, \beta_{2}^{0}}(x)=\frac{1}{\sigma_{\beta_{1}^{0}}\left(1-\rho^{2}\right)} \phi & \left(\frac{x}{\sigma_{\beta_{1}^{0}} \sqrt{1-\rho^{2}}}+\frac{\sqrt{n} \beta_{2}^{0} \rho}{\sigma_{\beta_{2}^{0}} \sqrt{1-\rho^{2}}}\right) \times \Delta\left(\frac{\sqrt{n} \beta_{2}^{0}}{\sigma_{\beta_{2}^{0}}}, \sqrt{\log n}\right) \\
& +\frac{\phi\left(\frac{x}{\sigma_{\beta_{1}^{0}}}\right)}{\sigma_{\beta_{1}^{0}}}\left(1-\Delta\left(\frac{\sqrt{n} \beta_{2}^{0} / \sigma_{\beta_{2}^{0}}+\rho x / \sigma_{\beta_{1}^{0}}}{\sqrt{1-\rho^{2}}}, \frac{\sqrt{\log n}}{\sqrt{1-\rho^{2}}}\right)\right),
\end{aligned}
$$

where $\phi(\cdot)$ denotes the density function of a standard normal, and $\sigma_{\beta_{1}^{0}}$ and $\sigma_{\beta_{2}^{0}}$ 
are respectively defined by

$$
\sigma^{2}\left(\boldsymbol{X}^{\top} \boldsymbol{X} / n\right)^{-1}=\left(\begin{array}{cc}
\sigma_{\beta_{1}^{0}}^{2} & \sigma_{\beta_{1}^{0}, \beta_{2}^{0}} \\
\sigma_{\beta_{1}^{0}, \beta_{2}^{0}} & \sigma_{\beta_{2}^{0}}^{2}
\end{array}\right)
$$

Moreover, $\rho$ is the correlation coefficient between the OLS estimators for $\beta_{1}^{0}$ and $\beta_{2}^{0}$ under the full model, which is given by $\rho=\sigma_{\beta_{1}^{0}, \beta_{2}^{0}} /\left(\sigma_{\beta_{1}^{0}} \sigma_{\beta_{2}^{0}}\right)$, where $\sigma_{\beta_{1}^{0}}, \sigma_{\beta_{2}^{0}}$ and $\sigma_{\beta_{1}^{0}, \beta_{2}^{0}}$ are given in $(6)$. In addition, the function $\Delta(\cdot, \cdot)$ is defined by $\Delta(a, b)=\Phi(a+b)-\Phi(a-b)$, where $\Phi(\cdot)$ is the CDF of a standard normal.

From $(5)$, we see that the finite-sample density function of $\sqrt{n}\left(\widetilde{\beta}_{1, \mathrm{PMS}}-\beta_{1}^{0}\right)$ is the sum of two components, respectively representing the density of $\sqrt{n}\left(\widehat{\beta}_{1 \cdot \mathbf{R}}-\right.$ $\left.\beta_{1}^{0}\right)$ and $\sqrt{n}\left(\widehat{\beta}_{1} \cdot \mathbf{U}-\beta_{1}^{0}\right)$, multiplied by the corresponding model selection probability. Clearly, although the density of $\sqrt{n}\left(\widetilde{\beta}_{1, \mathrm{PMS}}-\beta_{1}^{0}\right)$ does not depend on $\beta_{1}^{0}$, it hinges on the other unknown parameter $\beta_{2}^{0}$. With further evidence from simulations, Leeb and Pötscher demonstrate [ibid., Figure 2] that finite-sample distribution of $\sqrt{n}\left(\widetilde{\beta}_{1, \mathrm{PMS}}-\beta_{1}^{0}\right)$ is far away from Gaussian distribution for certain values of $\beta_{2}^{0}$.

As such, the equation (5) and accompanying simulations indicate that under consistent model selection framework, despite the fact that $\sqrt{n}\left(\widetilde{\beta}_{1, \mathrm{PMS}}-\beta_{1}^{0}\right)$ is asymptotically normal for a fixed true regression coefficient $\left(\beta_{1}^{0}, \beta_{2}^{0}\right)$, the convergence of $\sqrt{n}\left(\widetilde{\beta}_{1, \mathrm{PMS}}-\beta_{1}^{0}\right)$ to its limit is non-uniform with respect to the unknown parameters $\beta_{1}^{0}$ and $\beta_{2}^{0}$. Indeed, Pötscher (1991) concisely remarks that consistent model selectors usually produce super-efficient estimators, where non-uniformity is observed in the convergence of finite-sample distributions to their asymptotic equivalents.

Until this stage, it seems that irrespective of consistent or inconsistent model selectors, both the asymptotic conditional and unconditional distributions of the PMS estimator fail to approximate their finite-sample counterparts in a uniform fashion.

Therefore, a natural question to ask is that can the conditional and unconditional finite-sample distribution of $\widetilde{\boldsymbol{\beta}}_{\mathrm{PMS}}$ be estimated in a uniformly consistent manner?

Unfortunately, Leeb \& Pötscher (2006, 2008) assert that the answer to the above question is negative. Using sequential hypothesis testing as the model 
selector, Leeb \& Pötscher (2006) consider the existence of a uniformly consistent estimator of, the conditional finite-sample distribution of, $\sqrt{n} \boldsymbol{T}\left(\widetilde{\boldsymbol{\beta}}_{\mathrm{PMS}}-\boldsymbol{\beta}^{0}\right)$, where $\boldsymbol{T}$ is a deterministic $m \times p$ linear transformation matrix. For $\boldsymbol{x} \in \mathbb{R}^{m}$, such distribution function is denoted by $F_{n, \boldsymbol{\beta}^{0}, \sigma}(\cdot \mid \widehat{\boldsymbol{M}})$ and is defined as follows:

$F_{n, \boldsymbol{\beta}^{0}, \sigma}(\boldsymbol{x} \mid \widehat{\boldsymbol{M}})=\sum_{K=1}^{p} \mathbb{P}_{n, \boldsymbol{\beta}^{0}, \sigma}\left(\sqrt{n} \boldsymbol{T}\left(\widetilde{\boldsymbol{\beta}}_{\mathrm{PMS}}-\boldsymbol{\beta}^{0}\right) \leq \boldsymbol{x} \mid \widehat{\boldsymbol{M}}=\boldsymbol{M}_{[K]}\right) \mathbb{1}\left(\widehat{\boldsymbol{M}}=\boldsymbol{M}_{[K]}\right)$

Although a consistent estimator of $F_{n, \boldsymbol{\beta}^{0}, \sigma}(\cdot \mid \widehat{\boldsymbol{M}})$ can be constructed [ibid., Proposition 2.1 and Corollary 2.2] with relative ease, the authors show [ibid., Theorem 2.3] the non-existence of a uniformly consistent estimator of $F_{n, \boldsymbol{\beta}^{0}, \sigma}(\cdot \mid \widehat{\boldsymbol{M}})$. In addition, the authors demonstrate [ibid., Theorems 3.1, 4.1, 4.2 and 4.5] that such non-existence can be extended to a large class of model selectors, including minimizing AIC. Leeb \& Pötscher (2008) derive [Theorems 2.3 and 3.1] an analogous result that a uniformly consistent estimator of, the unconditional finite-sample distribution of, $\sqrt{n} \boldsymbol{T}\left(\widetilde{\boldsymbol{\beta}}_{\mathrm{PMS}}-\boldsymbol{\beta}^{0}\right)$, does not exist. 


\section{S3. Properties of Coverage Probabilities of Naïve Confidence Intervals for Population-based Targets in Linear Regression Models}

In this section, we present a detailed survey of finite- and large-sample results of coverage probabilities attained by the naïve marginal and simultaneous confidence intervals for $\boldsymbol{\beta}^{0}$ in linear regression models. This section complements Section 4 of the main manuscript.

Using minimizing AIC and BIC as model selectors, Hurvich \& Tsai (1990) perform Monte Carlo simulations to ascertain the effects of data-driven model selection on the finite-sample conditional (on the order of selected submodels) and unconditional coverage probabilities of naïve simultaneous confidence region for $\boldsymbol{\beta}^{0}$. Their simulations show that both the conditional and unconditional coverage rates of naïve confidence intervals are lower than the nominal coverage rates. In extreme cases, these coverage rates can reach as low as zero.

Similar under-coverage but under a log-linear model is obtained by Regal \& Hook (1991) through Monte Carlo simulations as well. In their work, the coverage target is the size of a closed population and a candidate submodel is chosen by the backward stepwise procedure.

With exactly the same setting as Hurvich \& Tsai (1990), Zhang (1992) further shows both theoretically [ibid., Theorem 3] and through Monte Carlo simulations [ibid., Table 1] that if generalized final prediction error (Shibata 1984) is used as the model selector to choose a submodel from a collection of nested submodels, the naïve simultaneous confidence regions for $\boldsymbol{\beta}^{0}$, fail to achieve the nominal coverage rates asymptotically.

One salient feature of Hurvich \& Tsai (1990), Regal \& Hook (1991) and Zhang (1992) is that the true regression coefficient $\boldsymbol{\beta}^{0}$ is deterministic. However, with Hodge's example of a "super-efficient" estimator, Kabaila (1995) argues that fixing the true regression coefficient invalidates the asymptotic criterion for constructing valid post-selection confidence regions to cover $\boldsymbol{\beta}^{0}$. Mathematically, for some data-dependent simultaneous confidence region $\mathcal{S}_{n}$, the criterion

$$
\lim _{n \rightarrow \infty} \mathbb{P}\left(\boldsymbol{\beta}^{0} \in \mathcal{S}_{n}\right)=1-\alpha,
$$

is illegitimate. As such, he proposes the standard for constructing asymptotically 
valid post-selection confidence region $\widetilde{\mathcal{S}}_{n}$ as

$$
\lim _{n \rightarrow \infty} \inf _{\boldsymbol{\beta}^{0} \in \Theta} \mathbb{P}\left(\boldsymbol{\beta}^{0} \in \widetilde{\mathcal{S}}_{n}\right)=1-\alpha,
$$

where $\Theta$ is the parametric space of all the possible values of $\boldsymbol{\beta}^{0}$. In words, an asymptotically valid post-selection confidence region should achieve the nominal minimum coverage rate over $\Theta$.

Moreover, Kabaila shows via an example that the confidence region yielded by the consistent model selectors usually satisfies (7) but not (8). Indeed, using minimum BIC as an instance of consistent model selectors, Leeb \& Pötscher (2005) explicitly formulate the finite-sample coverage probability for the parameter of interest attained by naïve confidence intervals (see equation (4) for the settings). They justify both theoretically and through simulations [ibid., Figure $3]$ that for certain values of $\boldsymbol{\beta}^{0}$, the finite-sample minimum coverage probability of naïve confidence intervals for the parameter of interest actually converges to zero.

In light of the previous discussions, it is advisable to investigate the effects of data-driven model selection on the minimal coverage probability achieved by the naïve confidence intervals. On the other hand, we should be aware that the analytic and simulation results in Hurvich \& Tsai (1990), Regal \& Hook (1991), Zhang (1992) are still valid as the coverage probability for any fixed regression coefficient provides an upper bound for the minimal coverage probability.

To quantify the effects of model selection on the minimal coverage rates of naïve marginal confidence intervals, Kabaila (2005) proposes [ibid., Section 2] a novel Monte Carlo simulation algorithm based on conditioning for variance reduction. This algorithm assesses the coverage probabilities of naïve confidence intervals for a given parameter value, where the coverage target is a linear combination of components of $\boldsymbol{\beta}^{0}$. The new algorithm is less computationally intensive than the brute force Monte Carlo algorithm and is capable of searching over some specified parameter space for a relatively small coverage probability. The output of this algorithm serves as an upper bound for the minimal coverage probability.

Kabaila \& Leeb (2006) analytically derive [ibid., Theorem 1] an upper bound of the large-sample minimal coverage probabilities of naïve confidence intervals 
for a given regression coefficient. Assuming the parameter of interest is $\beta_{1}^{0}$, the authors show that this upper bound is given by

$$
\begin{aligned}
\Psi_{\alpha}\left(\rho_{p}, c\right)= & \inf _{x \in \mathbb{R}} \Psi\left(x, \rho_{p}, c\right)=\inf _{x \in \mathbb{R}}\left[\Delta\left(\frac{\rho_{p} x}{\sqrt{1-\rho_{p}^{2}}}, z_{\alpha / 2}\right) \Delta(x, c)\right. \\
& \left.+\int_{-z_{\alpha / 2}}^{z_{\alpha / 2}}\left(1-\Delta\left(\frac{x+\rho_{p} z}{\sqrt{1-\rho_{p}^{2}}}, \frac{c}{\sqrt{1-\rho_{p}^{2}}}\right)\right) \phi(z) d z\right]
\end{aligned}
$$

where $\Delta(a, b)$ and $\phi$ are defined in $(5), z_{\alpha / 2}$ denotes the $(1-\alpha / 2)^{\text {th }}$ percentile of a standard normal and $c$ is a model selector-dependent constant which is to be specified. Moreover, $\rho_{p}$ is defined by

$$
\rho_{p}=\lim _{n \rightarrow \infty} \frac{\left[\left(\boldsymbol{X}^{\top} \boldsymbol{X}\right)^{-1}\right]_{1, p}}{\sqrt{\left[\left(\boldsymbol{X}^{\top} \boldsymbol{X}\right)^{-1}\right]_{1,1}\left[\left(\boldsymbol{X}^{\top} \boldsymbol{X}\right)^{-1}\right]_{p, p}}} .
$$

The authors assert [ibid., Figure 1] that the upper bound in (9) has an inverse relationship with $\left|\rho_{p}\right|$. Moreover, when using minimum AIC as the model selector and setting the type I error $\alpha=0.05$, the authors show that the constant $c$ in (9) is $\sqrt{2}$ and this upper bound approaches 0.11 as $\left|\rho_{p}\right|$ goes to 1 . Furthermore, they show that this upper bound applies to a large class of model selectors with different constants $c$.

An analogue of the upper bound in (9) for the finite-sample minimal coverage probabilities of naïve confidence intervals is given by Kabaila \& Giri (2009), where model selectors such as minimizing AIC, BIC and Mallows's $C_{p}$ [Mallows (1973)], maximizing adjusted $R^{2}$ and sequential $t$-testing are considered.

\section{S4. Exact Post-Selection Inference (EPoSI): Supplementary Formula}

In this section, we present the explicit forms of (1) the affine matrix $\boldsymbol{A}(\boldsymbol{M}, \boldsymbol{s})$ and vector $\boldsymbol{B}(\boldsymbol{M}, \boldsymbol{s})$ corresponding to LASSO with a fixed tuning parameter $\lambda$; (2) the truncation points $\mathcal{V}^{-}(\boldsymbol{Y}), \mathcal{V}^{+}(\boldsymbol{Y})$ and $\mathcal{V}^{0}(\boldsymbol{Y})$. These formulations complement the key steps in the construction of the EPoSI confidence intervals in Section 5.2.1 of the main manuscript.

When LASSO is used as the model selector with a fixed tuning parameter $\lambda$, the affine matrix $\boldsymbol{A}(\boldsymbol{M}, \boldsymbol{s}) \in \mathbb{R}^{(2 p-|M|) \times n}$ and vector $\boldsymbol{B}(\boldsymbol{M}, \boldsymbol{s}) \in \mathbb{R}^{(2 p-|M|) \times 1}$ 
are respectively given by

$$
A(M, s)=\left[\begin{array}{l}
A_{0}(M, s) \\
A_{1}(M, s)
\end{array}\right] \text { and } B(M, s)=\left[\begin{array}{l}
B_{0}(M, s) \\
B_{1}(M, s)
\end{array}\right],
$$

where

$$
\begin{aligned}
& \boldsymbol{A}_{\mathbf{0}}(\boldsymbol{M}, \boldsymbol{s})=\frac{1}{\lambda}\left[\begin{array}{c}
\boldsymbol{X}_{-M}^{\top}\left(\boldsymbol{I}-\boldsymbol{P}_{\boldsymbol{M}}\right) \\
-\boldsymbol{X}_{-M}^{\top}\left(\boldsymbol{I}-\boldsymbol{P}_{M}\right)
\end{array}\right], \\
& \boldsymbol{A}_{\mathbf{1}}(\boldsymbol{M}, \boldsymbol{s})=-\operatorname{diag}(\boldsymbol{s})\left(\boldsymbol{X}_{\boldsymbol{M}}^{\top} \boldsymbol{X}_{\boldsymbol{M}}\right)^{-1} \boldsymbol{X}_{\boldsymbol{M}}^{\top}, \\
& \boldsymbol{B}_{\mathbf{0}}(\boldsymbol{M}, \boldsymbol{s})=\left[\begin{array}{c}
\mathbf{1}-\boldsymbol{X}_{-M}^{\top}\left(\boldsymbol{X}_{\boldsymbol{M}}^{\top}\right)^{+} \boldsymbol{s} \\
\mathbf{1}+\boldsymbol{X}_{-M}^{\top}\left(\boldsymbol{X}_{\boldsymbol{M}}^{\top}\right)^{+} \boldsymbol{s}
\end{array}\right], \\
& \boldsymbol{B}_{\mathbf{1}}(\boldsymbol{M}, \boldsymbol{s})=-\lambda \operatorname{diag}(\boldsymbol{s})\left(\boldsymbol{X}_{\boldsymbol{M}}^{\top} \boldsymbol{X}_{\boldsymbol{M}}\right)^{-1} \boldsymbol{s} .
\end{aligned}
$$

Here, $\boldsymbol{X}_{-\boldsymbol{M}} \in \mathbb{R}^{n \times(p-|\boldsymbol{M}|)}$ is the submatrix of the full design matrix $\boldsymbol{X}$ with columns index by $\{1, \ldots, p\} \backslash \boldsymbol{M}, \boldsymbol{I} \in \mathbb{R}^{n \times n}$ is the identity matrix, $\boldsymbol{P}_{\boldsymbol{M}} \in$ $\mathbb{R}^{n \times n}$ is the projection matrix onto the column span of $\boldsymbol{X}_{\boldsymbol{M}}$ given by $\boldsymbol{P}_{\boldsymbol{M}}=$ $\boldsymbol{X}_{\boldsymbol{M}}\left(\boldsymbol{X}_{\boldsymbol{M}}^{\top} \boldsymbol{X}_{\boldsymbol{M}}\right)^{-1} \boldsymbol{X}_{\boldsymbol{M}}^{\top}, \operatorname{diag}(\boldsymbol{s})$ is the $|\boldsymbol{M}| \times|\boldsymbol{M}|$ diagonal matrix with diagonal elements given by $\boldsymbol{s}$, and $\boldsymbol{X}_{\boldsymbol{M}}^{+} \in \mathbb{R}^{|\boldsymbol{M}| \times n}$ is the pseudo-inverse of $\boldsymbol{X}_{\boldsymbol{M}}$ given by $\boldsymbol{X}_{M}^{+}=\left(\boldsymbol{X}_{\boldsymbol{M}}^{\top} \boldsymbol{X}_{M}\right)^{-1} \boldsymbol{X}_{M}^{\top}$.

The truncation points $\mathcal{V}^{-}(\boldsymbol{Y}), \mathcal{V}^{+}(\boldsymbol{Y})$ and $\mathcal{V}^{0}(\boldsymbol{Y})$ are formulated as

$$
\begin{aligned}
\mathcal{V}^{-}(\boldsymbol{Y}) & =\max _{j: \boldsymbol{w}_{j}<0} \frac{\boldsymbol{B}(\boldsymbol{M}, \boldsymbol{s})_{j}-(\boldsymbol{A}(\boldsymbol{M}, \boldsymbol{s}) \boldsymbol{Y})_{j}+\boldsymbol{w}_{j} \boldsymbol{\eta}_{j}^{\top} \boldsymbol{Y}}{\boldsymbol{w}_{j}}, \\
\mathcal{V}^{-}(\boldsymbol{Y}) & =\min _{j: \boldsymbol{w}_{j}>0} \frac{\boldsymbol{B}(\boldsymbol{M}, \boldsymbol{s})_{j}-(\boldsymbol{A}(\boldsymbol{M}, s) \boldsymbol{Y})_{j}+\boldsymbol{w}_{j} \boldsymbol{\eta}_{j}^{\top} \boldsymbol{Y}}{\boldsymbol{w}_{j}}, \\
\mathcal{V}^{0}(\boldsymbol{Y}) & =\min _{j: \boldsymbol{w}_{j}=0} \boldsymbol{B}(\boldsymbol{M}, \boldsymbol{s})_{j}-(\boldsymbol{A}(\boldsymbol{M}, \boldsymbol{s}) \boldsymbol{Y})_{j}, \\
\boldsymbol{w}_{j} & =\frac{\sigma^{2}\left(\boldsymbol{A}(\boldsymbol{M}, \boldsymbol{s}) \boldsymbol{\eta}_{j}\right)}{\sigma^{2} \boldsymbol{\eta}_{j}^{\top} \boldsymbol{\eta}_{j}}
\end{aligned}
$$

where $\boldsymbol{v}_{j}$ denotes the $j^{\text {th }}$ element of the vector $\boldsymbol{v}$ and the division of two vectors with the same dimensions is carried out by the component-wise division.

\section{S5. Additional Simulation Results}

Now, we provide simulation results in addition to Tables 3 to 5 as reported in Section 6.4 of the main manuscript. Each table presented here contains the em- 
pirical average coverage probability $\left(\mathrm{CP}_{j \cdot \widehat{M}}\right)$, and the empirical average length of confidence intervals $\left(\mathrm{L}_{j \cdot \widehat{M}}\right)$, for the projection-based regression coefficients $b_{j \cdot \widehat{M}}$ for $j \in M_{\text {true }}$. To be more specific, the simulation results shown in this section correspond to (1) the design matrices, $\boldsymbol{X}_{\mathrm{Tp}}$ and $\boldsymbol{X}_{\mathrm{Ep}}$, under Model I with dimension $n=40$ and $p=14$ (Section S5.1); (2) all five the design matrices under Models II, III and IV with dimension $n=40$ and $p=14$ (Sections S5.2 to S5.4); (3) all the five design matrices under Models I, II, III and IV with dimension $n=60$ and $p=18$ (Sections S5.5 to S5.8).

S5.1. $\quad$ Model I $(n=40, p=14)$

\begin{tabular}{|c|c|c|c|c|c|c|c|c|c|c|}
\hline \multirow[b]{3}{*}{$\begin{array}{l}\text { Top Selected } \\
\text { Submodels }\end{array}$} & \multicolumn{10}{|c|}{ Post-selection Confidence Intervals } \\
\hline & \multicolumn{2}{|c|}{ Naïve } & \multicolumn{2}{|c|}{ Scheffé } & \multicolumn{2}{|c|}{ PoSI } & \multicolumn{2}{|c|}{ EPoSI1 } & \multicolumn{2}{|c|}{ EPoSI2 } \\
\hline & $\mathrm{CP}_{1 \cdot \widehat{M}}$ & $\mathrm{~L}_{1 \cdot \widehat{M}}$ & $\mathrm{CP}_{1 \cdot \widehat{M}}$ & $\mathrm{~L}_{1 \cdot \widehat{\boldsymbol{M}}}$ & $\mathrm{CP}_{1 \cdot \widehat{M}}$ & $\mathrm{~L}_{1 \cdot \widehat{M}}$ & $\mathrm{CP}_{1 \cdot \widehat{M}}$ & $\mathrm{~L}_{1 \cdot \widehat{\boldsymbol{M}}}$ & $\mathrm{CP}_{1 \cdot \widehat{M}}$ & $\mathrm{~L}_{1 \cdot \widehat{M}}$ \\
\hline$\{1,2,3\}$ & .945 & 2.10 & 1.00 & 5.61 & .999 & 4.34 & .937 & 2.03 & .937 & 2.03 \\
\hline$\{1,2,3,4\}$ & .937 & 2.15 & 1.00 & 5.73 & .999 & 4.44 & .937 & 3.09 & .938 & 3.22 \\
\hline$\{1,2,3,5\}$ & .926 & 2.21 & 1.00 & 5.90 & 1.00 & 4.57 & .950 & 4.36 & .950 & 4.36 \\
\hline $\begin{array}{l}\text { Top Selected } \\
\text { Submodels }\end{array}$ & $\mathrm{CP}_{2 \cdot \widehat{M}}$ & $\mathrm{~L}_{2 \cdot \widehat{M}}$ & $\mathrm{CP}_{2 \cdot \widehat{M}}$ & $\mathrm{~L}_{2 \cdot \widehat{M}}$ & $\mathrm{CP}_{2 \cdot \widehat{M}}$ & $\mathrm{~L}_{2 \cdot \widehat{M}}$ & $\mathrm{CP}_{2 \cdot \widehat{M}}$ & $\mathrm{~L}_{2 \cdot \widehat{M}}$ & $\mathrm{CP}_{2 \cdot \widehat{M}}$ & $\mathrm{~L}_{2 \cdot \widehat{\boldsymbol{M}}}$ \\
\hline ( $\{1,2,3\}$ & .947 & 2.36 & 1.00 & 6.30 & 1.00 & 4.88 & .939 & 2.28 & .939 & 2.31 \\
\hline$\{1,2,3,4\}$ & .939 & 2.37 & 1.00 & 6.33 & 1.00 & 4.90 & .932 & 2.43 & .930 & 2.54 \\
\hline$\{1,2,3,5\}$ & .954 & 2.39 & 1.00 & 6.38 & 1.00 & 4.94 & .947 & 2.76 & .949 & 2.94 \\
\hline $\begin{array}{l}\text { Top Selected } \\
\text { Submodels }\end{array}$ & $\mathrm{CP}_{3 \cdot \widehat{M}}$ & $\mathrm{~L}_{3 \cdot \widehat{M}}$ & $\mathrm{CP}_{3 \cdot \widehat{M}}$ & $\mathrm{~L}_{3 \cdot \widehat{M}}$ & $\mathrm{CP}_{3 \cdot \widehat{M}}$ & $\mathrm{~L}_{3 \cdot \widehat{M}}$ & $\mathrm{CP}_{3 \cdot \widehat{M}}$ & $\mathrm{~L}_{3 \cdot \widehat{M}}$ & $\mathrm{CP}_{3 \cdot \widehat{M}}$ & $\mathrm{~L}_{3 \cdot \widehat{M}}$ \\
\hline$\{1,2,3\}$ & .951 & 2.36 & 1.00 & 6.32 & 1.00 & 4.89 & .944 & 2.31 & .944 & 2.32 \\
\hline$\{1,2,3,4\}$ & .922 & 3.28 & 1.00 & 8.76 & 1.00 & 6.78 & .911 & 3.17 & .963 & 9.04 \\
\hline$\{1,2,3,5\}$ & .917 & 2.92 & 1.00 & 7.79 & 1.00 & 6.03 & .965 & 6.89 & .972 & 7.76 \\
\hline
\end{tabular}

$C P_{j \cdot \widehat{M}}$ and $L_{j \cdot \widehat{M}}, j=1,2$ and 3, of post-selection confidence intervals based on Model I and $\boldsymbol{X}_{T p}$. 


\begin{tabular}{|c|c|c|c|c|c|c|c|c|c|c|}
\hline \multirow[b]{3}{*}{$\begin{array}{l}\text { Top Selected } \\
\text { Submodels }\end{array}$} & \multicolumn{10}{|c|}{ Post-selection Confidence Intervals } \\
\hline & \multicolumn{2}{|c|}{ Naïve } & \multicolumn{2}{|c|}{ Scheffé } & \multicolumn{2}{|c|}{ PoSI } & \multicolumn{2}{|c|}{ EPoSI1 } & \multicolumn{2}{|c|}{ EPoSI2 } \\
\hline & $\mathrm{CP}_{1 \cdot \widehat{M}}$ & $\mathrm{~L}_{1 \cdot \widehat{M}}$ & $\mathrm{CP}_{1 \cdot \widehat{M}}$ & $\mathrm{~L}_{1 \cdot \widehat{M}}$ & $\mathrm{CP}_{1 \cdot \widehat{M}}$ & $\mathrm{~L}_{1 \cdot \widehat{M}}$ & $\mathrm{CP}_{1 \cdot \widehat{M}}$ & $\mathrm{~L}_{1 \cdot \widehat{M}}$ & $\mathrm{CP}_{1 \cdot \widehat{M}}$ & $\mathrm{~L}_{1 \cdot \widehat{M}}$ \\
\hline$\{1,3\}$ & .945 & .932 & 1.00 & 2.49 & 1.00 & 1.89 & .938 & .917 & .938 & .917 \\
\hline$\{1,2,3\}$ & .947 & 1.14 & 1.00 & 3.03 & 1.00 & 2.30 & .938 & 1.10 & .969 & 2.86 \\
\hline$\{1,3,13\}$ & .938 & .950 & 1.00 & 2.54 & 1.00 & 1.93 & .927 & .945 & .950 & 1.69 \\
\hline $\begin{array}{l}\text { Top Selected } \\
\text { Submodels }\end{array}$ & $\mathrm{CP}_{2 \cdot \widehat{M}}$ & $\mathrm{~L}_{2 \cdot \widehat{M}}$ & $\mathrm{CP}_{2 \cdot \widehat{M}}$ & $\mathrm{~L}_{2 \cdot \widehat{M}}$ & $\mathrm{CP}_{2 \cdot \widehat{M}}$ & $\mathrm{~L}_{2 \cdot \widehat{M}}$ & $\mathrm{CP}_{2 \cdot \widehat{M}}$ & $\mathrm{~L}_{2 \cdot \widehat{M}}$ & $\mathrm{CP}_{2 \cdot \widehat{M}}$ & $\mathrm{~L}_{2 \cdot \widehat{M}}$ \\
\hline$\{1,2,3\}$ & .933 & 1.28 & 1.00 & 3.41 & .999 & 2.59 & .923 & 1.23 & .961 & 4.23 \\
\hline$\{1,2,3,13\}$ & .974 & 1.31 & 1.00 & 3.50 & 1.00 & 2.65 & .970 & 1.48 & .977 & 5.52 \\
\hline$\{1,2,3,6\}$ & .958 & 1.29 & 1.00 & 3.45 & 1.00 & 2.62 & .956 & 1.57 & .976 & $4.96 \mathrm{~s}$ \\
\hline $\begin{array}{l}\text { Top Selected } \\
\text { Submodels }\end{array}$ & $\mathrm{CP}_{3 \cdot \widehat{M}}$ & $\mathrm{~L}_{3 \cdot \widehat{M}}$ & $\mathrm{CP}_{3 \cdot \widehat{M}}$ & $\mathrm{~L}_{3 \cdot \widehat{M}}$ & $\mathrm{CP}_{3 \cdot \widehat{M}}$ & $\mathrm{~L}_{3 \cdot \widehat{M}}$ & $\mathrm{CP}_{3 \cdot \widehat{M}}$ & $\mathrm{~L}_{3 \cdot \widehat{M}}$ & $\mathrm{CP}_{3 \cdot \widehat{M}}$ & $\mathrm{~L}_{3 \cdot \widehat{M}}$ \\
\hline$\{1,3\}$ & .951 & .932 & 1.00 & 2.49 & 1.00 & 1.89 & .943 & .903 & .943 & .914 \\
\hline$\{1,2,3\}$ & .939 & 1.06 & 1.00 & 2.82 & 1.00 & 2.14 & .933 & 1.02 & .951 & 2.44 \\
\hline$\{1,3,13\}$ & .911 & 1.03 & 1.00 & 2.74 & .998 & 2.08 & .906 & 1.05 & .934 & 2.54 \\
\hline
\end{tabular}

$C P_{j \cdot \widehat{M}}$ and $L_{j \cdot \widehat{M}}, j=1,2$ and 3, of post-selection confidence intervals based on Model I and $\boldsymbol{X}_{E p}$. 
S5.2. $\quad$ Model II $(n=40, p=14)$

\begin{tabular}{|c|c|c|c|c|c|c|c|c|c|c|}
\hline & \multicolumn{10}{|c|}{ Post-selection Confidence Intervals } \\
\hline & \multicolumn{2}{|c|}{ Naïve } & \multicolumn{2}{|c|}{ Scheffé } & \multicolumn{2}{|c|}{ PoSI } & \multicolumn{2}{|c|}{ EPoSI1 } & \multicolumn{2}{|c|}{ EPoSI2 } \\
\hline $\begin{array}{c}\text { Top Selected } \\
\text { Submodels }\end{array}$ & $\mathrm{CP}_{1 \cdot \widehat{M}}$ & $\mathrm{~L}_{1 \cdot \widehat{M}}$ & $\mathrm{CP}_{1 \cdot \widehat{M}}$ & $\mathrm{~L}_{1 \cdot \widehat{M}}$ & $\mathrm{CP}_{1 \cdot \widehat{M}}$ & $\mathrm{~L}_{1 \cdot \widehat{M}}$ & $\mathrm{CP}_{1 \cdot \widehat{M}}$ & $\mathrm{~L}_{1 \cdot \widehat{M}}$ & $\mathrm{CP}_{1 \cdot \widehat{M}}$ & $\mathrm{~L}_{1 \cdot \widehat{M}}$ \\
\hline$\{1,2,3,4,5\}$ & .931 & 2.12 & 1.00 & 5.66 & 1.00 & 4.38 & .929 & 2.05 & .929 & 2.05 \\
\hline$\{1,2,3,4,5,11\}$ & .909 & 2.19 & 1.00 & 5.84 & 1.00 & 4.52 & .906 & 2.13 & .928 & 3.65 \\
\hline$\{1,2,3,4,5,7\}$ & .922 & 2.09 & 1.00 & 5.58 & .996 & 4.32 & .902 & 2.02 & .908 & 2.06 \\
\hline $\begin{array}{l}\text { Top Selected } \\
\text { Submodels }\end{array}$ & $\mathrm{CP}_{2 \cdot \widehat{M}}$ & $\mathrm{~L}_{2 \cdot \widehat{M}}$ & $\mathrm{CP}_{2 \cdot \widehat{M}}$ & $\mathrm{~L}_{2 \cdot \widehat{M}}$ & $\mathrm{CP}_{2 \cdot \widehat{M}}$ & $\mathrm{~L}_{2 \cdot \widehat{M}}$ & $\mathrm{CP}_{2 \cdot \widehat{M}}$ & $\mathrm{~L}_{2 \cdot \widehat{M}}$ & $\mathrm{CP}_{2 \cdot \widehat{M}}$ & $\mathrm{~L}_{2 \cdot \widehat{M}}$ \\
\hline$\{1,2,3,4,5\}$ & .949 & 2.12 & 1.00 & 5.65 & .998 & 4.38 & .939 & 2.04 & .941 & 2.05 \\
\hline$\{1,2,3,4,5,11\}$ & .930 & 2.12 & 1.00 & 5.65 & 1.00 & 4.38 & .923 & 2.08 & .939 & 2.80 \\
\hline$\{1,2,3,4,5,7\}$ & .959 & 2.09 & 1.00 & 5.57 & 1.00 & 4.32 & .952 & 2.02 & .958 & 2.05 \\
\hline $\begin{array}{l}\text { Top Selected } \\
\text { Submodels }\end{array}$ & $\mathrm{CP}_{3 \cdot \widehat{M}}$ & $\mathrm{~L}_{3 \cdot \widehat{M}}$ & $\mathrm{CP}_{3 \cdot \widehat{M}}$ & $\mathrm{~L}_{3 \cdot \widehat{M}}$ & $\mathrm{CP}_{3 \cdot \widehat{M}}$ & $\mathrm{~L}_{3 \cdot \widehat{M}}$ & $\mathrm{CP}_{3 \cdot \widehat{M}}$ & $\mathrm{~L}_{3 \cdot \widehat{M}}$ & $\mathrm{CP}_{3 \cdot \widehat{M}}$ & $\mathrm{~L}_{3 \cdot \widehat{M}}$ \\
\hline$\{1,2,3,4,5\}$ & .945 & 2.28 & 1.00 & 6.07 & 1.00 & 4.70 & .933 & 2.20 & .933 & 2.21 \\
\hline$\{1,2,3,4,5,11\}$ & .958 & 2.26 & 1.00 & 6.02 & 1.00 & 4.66 & .958 & 2.19 & .964 & 2.54 \\
\hline$\{1,2,3,4,5,7\}$ & .941 & 2.38 & 1.00 & 6.35 & 1.00 & 4.92 & .933 & 2.31 & .949 & 4.04 \\
\hline $\begin{array}{c}\text { Top Selected } \\
\text { Submodels }\end{array}$ & $\mathrm{CP}_{4 \cdot \widehat{M}}$ & $\mathrm{~L}_{4 \cdot \widehat{M}}$ & $\mathrm{CP}_{4 \cdot \widehat{M}}$ & $\mathrm{~L}_{4 \cdot \widehat{M}}$ & $\mathrm{CP}_{4 \cdot \widehat{M}}$ & $\mathrm{~L}_{4 \cdot \widehat{M}}$ & $\mathrm{CP}_{4 \cdot \widehat{M}}$ & $\mathrm{~L}_{4 \cdot \widehat{M}}$ & $\mathrm{CP}_{4 \cdot \widehat{M}}$ & $\mathrm{~L}_{4 \cdot \widehat{M}}$ \\
\hline 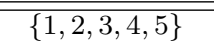 & .925 & 2.08 & 1.00 & 5.55 & 1.00 & 4.30 & .919 & 2.01 & .919 & 2.01 \\
\hline$\{1,2,3,4,5,11\}$ & .927 & 2.24 & 1.00 & 5.98 & 1.00 & 4.63 & .923 & 2.28 & .924 & 4.33 \\
\hline$\{1,2,3,4,5,7\}$ & .929 & 2.06 & 1.00 & 5.48 & 1.00 & 4.25 & .922 & 1.98 & .927 & 3.39 \\
\hline $\begin{array}{l}\text { Top Selected } \\
\text { Submodels }\end{array}$ & $\mathrm{CP}_{5 \cdot \widehat{M}}$ & $\mathrm{~L}_{5 \cdot \widehat{M}}$ & $\mathrm{CP}_{5 \cdot \widehat{M}}$ & $\mathrm{~L}_{5 \cdot \widehat{M}}$ & $\mathrm{CP}_{5 \cdot \widehat{M}}$ & $\mathrm{~L}_{5 \cdot \widehat{M}}$ & $\mathrm{CP}_{5 \cdot \widehat{M}}$ & $\mathrm{~L}_{5 \cdot \widehat{M}}$ & $\mathrm{CP}_{5 \cdot \widehat{M}}$ & $\mathrm{~L}_{5 \cdot \widehat{M}}$ \\
\hline$\{1,2,3,4,5\}$ & .951 & 2.68 & 1.00 & 7.15 & 1.00 & 5.54 & .949 & 2.59 & .949 & 2.63 \\
\hline$\{1,2,3,4,5,11\}$ & .948 & 2.77 & 1.00 & 7.37 & 1.00 & 5.71 & .937 & 2.67 & .939 & 4.61 \\
\hline$\{1,2,3,4,5,7\}$ & .959 & 2.92 & 1.00 & 7.77 & 1.00 & 6.02 & .955 & 2.81 & .969 & 5.53 \\
\hline
\end{tabular}

$C P_{j \cdot \widehat{M}}$ and $L_{j \cdot \widehat{M}}, j=1,2,3,4$ and 5, of post-selection confidence intervals based on Model

II and $\boldsymbol{X}_{E q}$. 


\begin{tabular}{|c|c|c|c|c|c|c|c|c|c|c|}
\hline & \multicolumn{10}{|c|}{ Post-selection Confidence Intervals } \\
\hline & \multicolumn{2}{|c|}{ Naïve } & \multicolumn{2}{|c|}{ Scheffé } & \multicolumn{2}{|c|}{ PoSI } & \multicolumn{2}{|c|}{ EPoSI1 } & \multicolumn{2}{|c|}{ EPoSI2 } \\
\hline $\begin{array}{l}\text { Top Selected } \\
\text { Submodels }\end{array}$ & $\mathrm{CP}_{1 \cdot \widehat{M}}$ & $\mathrm{~L}_{1 \cdot \widehat{M}}$ & $\mathrm{CP}_{1 \cdot \widehat{M}}$ & $\mathrm{~L}_{1 \cdot \widehat{M}}$ & $\mathrm{CP}_{1 \cdot \widehat{M}}$ & $\mathrm{~L}_{1 \cdot \widehat{M}}$ & $\mathrm{CP}_{1 \cdot \widehat{M}}$ & $\mathrm{~L}_{1 \cdot \widehat{M}}$ & $\mathrm{CP}_{1 \cdot \widehat{M}}$ & $\mathrm{~L}_{1 \cdot \widehat{M}}$ \\
\hline$\{1,2,3,4,5\}$ & .945 & 2.07 & 1.00 & 5.51 & 1.00 & 4.26 & .938 & 1.99 & .938 & 2.00 \\
\hline$\{1,2,3,4,5,6\}$ & .953 & 2.08 & 1.00 & 5.53 & 1.00 & 4.28 & .943 & 2.07 & .946 & 2.80 \\
\hline$\{1,2,3,4,5,7\}$ & .945 & 2.07 & 1.00 & 5.50 & 1.00 & 4.25 & .945 & 2.00 & .946 & 2.08 \\
\hline $\begin{array}{l}\text { Top Selected } \\
\text { Submodels }\end{array}$ & $\mathrm{CP}_{2 \cdot \widehat{M}}$ & $\mathrm{~L}_{2 \cdot \widehat{M}}$ & $\mathrm{CP}_{2 \cdot \widehat{M}}$ & $\mathrm{~L}_{2 \cdot \widehat{M}}$ & $\mathrm{CP}_{2 \cdot \widehat{M}}$ & $\mathrm{~L}_{2 \cdot \widehat{M}}$ & $\mathrm{CP}_{2 \cdot \widehat{M}}$ & $\mathrm{~L}_{2 \cdot \widehat{M}}$ & $\mathrm{CP}_{2 \cdot \widehat{M}}$ & $\mathrm{~L}_{2 \cdot \widehat{M}}$ \\
\hline$\{1,2,3,4,5\}$ & .955 & 2.84 & 1.00 & 7.58 & 1.00 & 5.86 & .948 & 2.74 & .948 & 2.79 \\
\hline$\{1,2,3,4,5,6\}$ & .955 & 2.82 & 1.00 & 7.53 & 1.00 & 5.82 & .945 & 2.75 & .949 & 3.02 \\
\hline$\{1,2,3,4,5,7\}$ & .949 & 2.84 & 1.00 & 7.57 & 1.00 & 5.86 & .942 & 2.74 & .943 & 2.86 \\
\hline $\begin{array}{l}\text { Top Selected } \\
\text { Submodels }\end{array}$ & $\mathrm{CP}_{3 \cdot \widehat{\boldsymbol{M}}}$ & $\mathrm{L}_{3 \cdot \widehat{M}}$ & $\mathrm{CP}_{3 \cdot \widehat{M}}$ & $\mathrm{~L}_{3 \cdot \widehat{M}}$ & $\mathrm{CP}_{3 \cdot \widehat{M}}$ & $\mathrm{~L}_{3 \cdot \widehat{M}}$ & $\mathrm{CP}_{3 \cdot \widehat{M}}$ & $\mathrm{~L}_{3 \cdot \widehat{M}}$ & $\mathrm{CP}_{3 \cdot \widehat{M}}$ & $\mathrm{~L}_{3 \cdot \widehat{M}}$ \\
\hline$\{1,2,3,4,5\}$ & .941 & 2.78 & 1.00 & 7.41 & 1.00 & 5.73 & .935 & 2.68 & .935 & 2.76 \\
\hline$\{1,2,3,4,5,6\}$ & .950 & 2.78 & 1.00 & 7.40 & 1.00 & 5.72 & .940 & 2.68 & .946 & 3.51 \\
\hline$\{1,2,3,4,5,7\}$ & .958 & 2.78 & 1.00 & 7.40 & 1.00 & 5.72 & .957 & 2.68 & .958 & 2.87 \\
\hline $\begin{array}{l}\text { Top Selected } \\
\text { Submodels }\end{array}$ & $\mathrm{CP}_{4 \cdot \widehat{M}}$ & $\mathrm{~L}_{4 \cdot \widehat{M}}$ & $\mathrm{CP}_{4 \cdot \widehat{M}}$ & $\mathrm{~L}_{4 \cdot \widehat{M}}$ & $\mathrm{CP}_{4 \cdot \widehat{M}}$ & $\mathrm{~L}_{4 \cdot \widehat{M}}$ & $\mathrm{CP}_{4 \cdot \widehat{M}}$ & $\mathrm{~L}_{4 \cdot \widehat{M}}$ & $\mathrm{CP}_{4 \cdot \widehat{M}}$ & $\mathrm{~L}_{4 \cdot \widehat{M}}$ \\
\hline$\{1,2,3,4,5\}$ & .944 & 2.77 & 1.00 & 7.37 & 1.00 & 5.70 & .935 & 2.67 & .935 & 2.72 \\
\hline$\{1,2,3,4,5,6\}$ & .945 & 2.75 & 1.00 & 7.32 & 1.00 & 5.66 & .941 & 2.65 & .943 & 3.00 \\
\hline$\{1,2,3,4,5,7\}$ & .947 & 2.78 & 1.00 & 7.41 & .998 & 5.73 & .945 & 2.72 & .950 & 3.46 \\
\hline $\begin{array}{l}\text { Top Selected } \\
\text { Submodels }\end{array}$ & $\mathrm{CP}_{5 \cdot \widehat{M}}$ & $\mathrm{~L}_{5 \cdot \widehat{M}}$ & $\mathrm{CP}_{5 \cdot \widehat{M}}$ & $\mathrm{~L}_{5 \cdot \widehat{M}}$ & $\mathrm{CP}_{5 \cdot \widehat{M}}$ & $\mathrm{~L}_{5 \cdot \widehat{M}}$ & $\mathrm{CP}_{5 \cdot \widehat{M}}$ & $\mathrm{~L}_{5 \cdot \widehat{M}}$ & $\mathrm{CP}_{5 \cdot \widehat{M}}$ & $\mathrm{~L}_{5 \cdot \widehat{M}}$ \\
\hline$\{1,2,3,4,5\}$ & .949 & 2.06 & 1.00 & 5.49 & 1.00 & 4.25 & .941 & 1.99 & .941 & 2.00 \\
\hline$\{1,2,3,4,5,6\}$ & .926 & 2.95 & 1.00 & 7.85 & 1.00 & 6.07 & .918 & 3.33 & .955 & 7.52 \\
\hline$\{1,2,3,4,5,7\}$ & .943 & 2.53 & 1.00 & 6.74 & 1.00 & 5.21 & .938 & 2.87 & .952 & 5.93 \\
\hline
\end{tabular}

$C P_{j \cdot \widehat{M}}$ and $L_{j \cdot \widehat{M}}, j=1,2,3,4$ and 5, of post-selection confidence intervals based on Model II and $\boldsymbol{X}_{T p}$. 


\begin{tabular}{|c|c|c|c|c|c|c|c|c|c|c|}
\hline & \multicolumn{10}{|c|}{ Post-selection Confidence Intervals } \\
\hline & \multicolumn{2}{|c|}{ Naïve } & \multicolumn{2}{|c|}{ Scheffé } & \multicolumn{2}{|c|}{ PoSI } & \multicolumn{2}{|c|}{ EPoSI1 } & \multicolumn{2}{|c|}{ EPoSI2 } \\
\hline $\begin{array}{c}\text { Top Selected } \\
\text { Submodels }\end{array}$ & $\mathrm{CP}_{1 \cdot \widehat{M}}$ & $\mathrm{~L}_{1 \cdot \widehat{M}}$ & $\mathrm{CP}_{1 \cdot \widehat{M}}$ & $\mathrm{~L}_{1 \cdot \widehat{M}}$ & $\mathrm{CP}_{1 \cdot \widehat{M}}$ & $\mathrm{~L}_{1 \cdot \widehat{M}}$ & $\mathrm{CP}_{1 \cdot \widehat{M}}$ & $\mathrm{~L}_{1 \cdot \widehat{M}}$ & $\mathrm{CP}_{1 \cdot \widehat{M}}$ & $\mathrm{~L}_{1 \cdot \widehat{M}}$ \\
\hline$\{1,3,5\}$ & .971 & .977 & 1.00 & 2.61 & .999 & 1.96 & .956 & 1.70 & .958 & 1.78 \\
\hline$\{1,3,4,5\}$ & .958 & .990 & 1.00 & 2.64 & 1.00 & 1.98 & .954 & 1.06 & .950 & 2.02 \\
\hline$\{1,3,5,12\}$ & .981 & .985 & 1.00 & 2.63 & 1.00 & 1.97 & .953 & 1.73 & .956 & 2.11 \\
\hline $\begin{array}{c}\text { Top Selected } \\
\text { Submodels }\end{array}$ & $\mathrm{CP}_{2 \cdot \widehat{M}}$ & $\mathrm{~L}_{2 \cdot \widehat{M}}$ & $\mathrm{CP}_{2 \cdot \widehat{M}}$ & $\mathrm{~L}_{2 \cdot \widehat{M}}$ & $\mathrm{CP}_{2 \cdot \widehat{M}}$ & $\mathrm{~L}_{2 \cdot \widehat{M}}$ & $\mathrm{CP}_{2 \cdot \widehat{M}}$ & $\mathrm{~L}_{2 \cdot \widehat{M}}$ & $\mathrm{CP}_{2 \cdot \widehat{M}}$ & $\mathrm{~L}_{2 \cdot \widehat{M}}$ \\
\hline$\{1,2,3,5\}$ & 0.00 & 1.29 & 1.00 & 3.44 & .750 & 2.58 & 0.00 & 1.13 & 1.00 & 4.59 \\
\hline$\{1,2,5,6,12\}$ & 0.00 & 1.43 & 1.00 & 3.80 & 0.00 & 2.85 & 1.00 & 2.25 & 1.00 & 2.25 \\
\hline$\{2,3,5,12\}$ & 0.00 & .895 & 1.00 & 2.39 & 0.00 & 1.79 & 1.00 & Inf & 1.00 & Inf \\
\hline $\begin{array}{c}\text { Top Selected } \\
\text { Submodels }\end{array}$ & $\mathrm{CP}_{3 \cdot \widehat{M}}$ & $\mathrm{~L}_{3 \cdot \widehat{M}}$ & $\mathrm{CP}_{3 \cdot \widehat{M}}$ & $\mathrm{~L}_{3 \cdot \widehat{M}}$ & $\mathrm{CP}_{3 \cdot \widehat{M}}$ & $\mathrm{~L}_{3 \cdot \widehat{M}}$ & $\mathrm{CP}_{3 \cdot \widehat{M}}$ & $\mathrm{~L}_{3 \cdot \widehat{\boldsymbol{M}}}$ & $\mathrm{CP}_{3 \cdot \widehat{M}}$ & $\mathrm{~L}_{3 \cdot \widehat{M}}$ \\
\hline$\{1,3,5\}$ & .976 & .963 & 1.00 & 2.57 & 1.00 & 1.93 & .959 & 1.64 & .960 & 1.78 \\
\hline$\{1,3,4,5\}$ & .935 & 1.04 & 1.00 & 2.78 & 1.00 & 2.08 & .925 & 1.10 & .957 & 2.51 \\
\hline$\{1,3,5,12\}$ & .992 & 1.00 & 1.00 & 2.68 & 1.00 & 2.01 & .987 & 1.24 & .969 & 2.84 \\
\hline $\begin{array}{c}\text { Top Selected } \\
\text { Submodels }\end{array}$ & $\mathrm{CP}_{4 \cdot \widehat{M}}$ & $\mathrm{~L}_{4 \cdot \widehat{M}}$ & $\mathrm{CP}_{4 \cdot \widehat{M}}$ & $\mathrm{~L}_{4 \cdot \widehat{M}}$ & $\mathrm{CP}_{4 \cdot \widehat{M}}$ & $\mathrm{~L}_{4 \cdot \widehat{M}}$ & $\mathrm{CP}_{4 \cdot \widehat{M}}$ & $\mathrm{~L}_{4 \cdot \widehat{M}}$ & $\mathrm{CP}_{4 \cdot \widehat{M}}$ & $\mathrm{~L}_{4 \cdot \widehat{M}}$ \\
\hline$\{1,3,4,5\}$ & .891 & 1.14 & 1.00 & 3.06 & 1.00 & 2.29 & .874 & 1.11 & .962 & 3.92 \\
\hline$\{1,3,4,5,12\}$ & .934 & 1.17 & 1.00 & 3.12 & .997 & 2.34 & .924 & 1.13 & .960 & 4.95 \\
\hline$\{1,3,4,5,14\}$ & .965 & 1.17 & 1.00 & 3.11 & 1.00 & 2.33 & .945 & 1.21 & .966 & 4.95 \\
\hline $\begin{array}{c}\text { Top Selected } \\
\text { Submodels }\end{array}$ & $\mathrm{CP}_{5 \cdot \widehat{M}}$ & $\mathrm{~L}_{5 \cdot \widehat{M}}$ & $\mathrm{CP}_{5 \cdot \widehat{M}}$ & $\mathrm{~L}_{5 \cdot \widehat{M}}$ & $\mathrm{CP}_{5 \cdot \widehat{M}}$ & $\mathrm{~L}_{5 \cdot \widehat{M}}$ & $\mathrm{CP}_{5 \cdot \widehat{M}}$ & $\mathrm{~L}_{5 \cdot \widehat{M}}$ & $\mathrm{CP}_{5 \cdot \widehat{M}}$ & $\mathrm{~L}_{5 \cdot \widehat{M}}$ \\
\hline$\{1,3,5\}$ & .969 & .921 & 1.00 & 2.46 & 1.00 & 1.85 & .958 & 1.70 & .958 & 1.71 \\
\hline$\{1,3,4,5\}$ & .922 & 1.09 & 1.00 & 2.90 & .999 & 2.18 & .916 & 1.05 & .969 & 3.04 \\
\hline$\{1,3,5,12\}$ & .980 & .930 & 1.00 & 2.48 & 1.00 & 1.86 & .974 & 1.04 & .964 & 2.05 \\
\hline
\end{tabular}

$C P_{j \cdot \widehat{M}}$ and $L_{j \cdot \widehat{M}}, j=1$, 2, 3, 4 and 5, of post-selection confidence intervals based on Model II and $\boldsymbol{X}_{E p}$. 


\begin{tabular}{|c|c|c|c|c|c|c|c|c|c|c|}
\hline & \multicolumn{10}{|c|}{ Post-selection Confidence Intervals } \\
\hline & \multicolumn{2}{|c|}{ Naïve } & \multicolumn{2}{|c|}{ Scheffé } & \multicolumn{2}{|c|}{ PoSI } & \multicolumn{2}{|c|}{ EPoSI1 } & \multicolumn{2}{|c|}{ EPoSI2 } \\
\hline $\begin{array}{c}\text { Top Selected } \\
\text { Submodels }\end{array}$ & $\mathrm{CP}_{1 \cdot \widehat{M}}$ & $\mathrm{~L}_{1 \cdot \widehat{M}}$ & $\mathrm{CP}_{1 \cdot \widehat{M}}$ & $\mathrm{~L}_{1 \cdot \widehat{M}}$ & $\mathrm{CP}_{1 \cdot \widehat{M}}$ & $\mathrm{~L}_{1 \cdot \widehat{M}}$ & $\mathrm{CP}_{1 \cdot \widehat{M}}$ & $\mathrm{~L}_{1 \cdot \widehat{\boldsymbol{M}}}$ & $\mathrm{CP}_{1 \cdot \widehat{M}}$ & $\mathrm{~L}_{1 \cdot \widehat{M}}$ \\
\hline$\{1,2,3,4,5\}$ & .953 & 5.11 & 1.00 & 13.6 & 1.00 & 10.5 & .943 & 4.93 & .951 & 6.37 \\
\hline$\{1,2,3,4\}$ & .943 & 4.89 & 1.00 & 13.1 & 1.00 & 10.0 & .931 & 4.73 & 1.00 & 4.68 \\
\hline$\{1,2,3,5\}$ & .949 & 4.92 & 1.00 & 13.1 & 1.00 & 10.1 & .944 & 4.75 & 1.00 & 4.29 \\
\hline $\begin{array}{l}\text { Top Selected } \\
\text { Submodels }\end{array}$ & $\mathrm{CP}_{2 \cdot \widehat{M}}$ & $\mathrm{~L}_{2 \cdot \widehat{M}}$ & $\mathrm{CP}_{2 \cdot \widehat{M}}$ & $\mathrm{~L}_{2 \cdot \widehat{M}}$ & $\mathrm{CP}_{2 \cdot \widehat{M}}$ & $\mathrm{~L}_{2 \cdot \widehat{M}}$ & $\mathrm{CP}_{2 \cdot \widehat{M}}$ & $\mathrm{~L}_{2 \cdot \widehat{M}}$ & $\mathrm{CP}_{2 \cdot \widehat{M}}$ & $\mathrm{~L}_{2 \cdot \widehat{M}}$ \\
\hline$\{1,2,3,4,5\}$ & .945 & 5.11 & 1.00 & 13.6 & .999 & 10.5 & .937 & 4.93 & .963 & 6.55 \\
\hline$\{1,2,3,4\}$ & .944 & 4.89 & 1.00 & 13.1 & 1.00 & 10.0 & .946 & 4.74 & 1.00 & 5.00 \\
\hline$\{1,2,3,5\}$ & .959 & 4.92 & 1.00 & 13.1 & 1.00 & 10.1 & .955 & 4.75 & .667 & 5.20 \\
\hline $\begin{array}{c}\text { Top Selected } \\
\text { Submodels }\end{array}$ & $\mathrm{CP}_{3 \cdot \widehat{M}}$ & $\mathrm{~L}_{3 \cdot \widehat{M}}$ & $\mathrm{CP}_{3 \cdot \widehat{M}}$ & $\mathrm{~L}_{3 \cdot \widehat{M}}$ & $\mathrm{CP}_{3 \cdot \widehat{M}}$ & $\mathrm{~L}_{3 \cdot \widehat{M}}$ & $\mathrm{CP}_{3 \cdot \widehat{M}}$ & $\mathrm{~L}_{3 \cdot \widehat{\boldsymbol{M}}}$ & $\mathrm{CP}_{3 \cdot \widehat{M}}$ & $\mathrm{~L}_{3 \cdot \widehat{M}}$ \\
\hline$\{1,2,3,4,5\}$ & .966 & 5.11 & 1.00 & 13.6 & 1.00 & 10.5 & .960 & 4.93 & .976 & 6.23 \\
\hline$\{1,2,3,4\}$ & .955 & 4.89 & 1.00 & 13.1 & 1.00 & 10.0 & .945 & 4.73 & 1.00 & 4.73 \\
\hline$\{1,2,3,5\}$ & .969 & 4.92 & 1.00 & 13.1 & 1.00 & 10.1 & .966 & 4.75 & 1.00 & 5.20 \\
\hline $\begin{array}{l}\text { Top Selected } \\
\text { Submodels }\end{array}$ & $\mathrm{CP}_{4 \cdot \widehat{M}}$ & $\mathrm{~L}_{4 \cdot \widehat{M}}$ & $\mathrm{CP}_{4 \cdot \widehat{M}}$ & $\mathrm{~L}_{4 \cdot \widehat{M}}$ & $\mathrm{CP}_{4 \cdot \widehat{M}}$ & $\mathrm{~L}_{4 \cdot \widehat{M}}$ & $\mathrm{CP}_{4 \cdot \widehat{M}}$ & $\mathrm{~L}_{4 \cdot \widehat{M}}$ & $\mathrm{CP}_{4 \cdot \widehat{M}}$ & $\mathrm{~L}_{4 \cdot \widehat{M}}$ \\
\hline$\{1,2,3,4,5\}$ & .982 & 5.11 & 1.00 & 13.6 & 1.00 & 10.5 & .981 & 5.01 & .976 & 5.80 \\
\hline$\{1,2,3,4\}$ & .952 & 4.89 & 1.00 & 13.1 & 1.00 & 10.0 & .960 & 4.74 & 1.00 & 4.66 \\
\hline$\{1,2,4,5\}$ & .981 & 4.89 & 1.00 & 13.1 & 1.00 & 10.0 & .971 & 4.72 & 1.00 & 5.06 \\
\hline $\begin{array}{l}\text { Top Selected } \\
\text { Submodels }\end{array}$ & $\mathrm{CP}_{5 \cdot \widehat{M}}$ & $\mathrm{~L}_{5 \cdot \widehat{M}}$ & $\mathrm{CP}_{5 \cdot \widehat{M}}$ & $\mathrm{~L}_{5 \cdot \widehat{M}}$ & $\mathrm{CP}_{5 \cdot \widehat{M}}$ & $\mathrm{~L}_{5 \cdot \widehat{M}}$ & $\mathrm{CP}_{5 \cdot \widehat{M}}$ & $\mathrm{~L}_{5 \cdot \widehat{M}}$ & $\mathrm{CP}_{5 \cdot \widehat{M}}$ & $\mathrm{~L}_{5 \cdot \widehat{M}}$ \\
\hline$\{1,2,3,4,5\}$ & .961 & 5.11 & 1.00 & 13.6 & 1.00 & 10.5 & .961 & 5.07 & .939 & 5.56 \\
\hline$\{1,2,3,5\}$ & .974 & 4.92 & 1.00 & 13.1 & 1.00 & 10.1 & .967 & 4.75 & 1.00 & 4.31 \\
\hline$\{1,2,4,5\}$ & .976 & 4.89 & 1.00 & 13.1 & 1.00 & 10.0 & .993 & 4.71 & 1.00 & 4.73 \\
\hline
\end{tabular}

$C P_{j \cdot \widehat{M}}$ and $L_{j \cdot \widehat{M}}, j=1$, 2, 3, 4 and 5, of post-selection confidence intervals based on Model

II and $\boldsymbol{X}_{E c}$. 


\begin{tabular}{|c|c|c|c|c|c|c|c|c|c|c|}
\hline & \multicolumn{10}{|c|}{ Post-selection Confidence Intervals } \\
\hline & \multicolumn{2}{|c|}{ Naïve } & \multicolumn{2}{|c|}{ Scheffé } & \multicolumn{2}{|c|}{ PoSI } & \multicolumn{2}{|c|}{ EPoSI1 } & \multicolumn{2}{|c|}{ EPoSI2 } \\
\hline $\begin{array}{c}\text { Top Selected } \\
\text { Submodels }\end{array}$ & $\mathrm{CP}_{1 \cdot \widehat{M}}$ & $\mathrm{~L}_{1 \cdot \widehat{M}}$ & $\mathrm{CP}_{1 \cdot \widehat{M}}$ & $\mathrm{~L}_{1 \cdot \widehat{M}}$ & $\mathrm{CP}_{1 \cdot \widehat{M}}$ & $\mathrm{~L}_{1 \cdot \widehat{M}}$ & $\mathrm{CP}_{1 \cdot \widehat{M}}$ & $\mathrm{~L}_{1 \cdot \widehat{\boldsymbol{M}}}$ & $\mathrm{CP}_{1 \cdot \widehat{M}}$ & $\mathrm{~L}_{1 \cdot \widehat{M}}$ \\
\hline$\{1,3,4\}$ & .976 & 5.92 & 1.00 & 13.0 & 1.00 & 10.2 & .976 & 5.96 & .991 & 15.5 \\
\hline$\{1,4\}$ & .986 & 5.88 & 1.00 & 12.9 & 1.00 & 10.2 & .986 & 5.86 & .981 & 12.7 \\
\hline$\{1,2,3,4\}$ & .989 & 5.95 & 1.00 & 13.0 & 1.00 & 10.3 & .989 & 7.32 & .977 & 17.3 \\
\hline $\begin{array}{l}\text { Top Selected } \\
\text { Submodels }\end{array}$ & $\mathrm{CP}_{2 \cdot \widehat{M}}$ & $\mathrm{~L}_{2 \cdot \widehat{M}}$ & $\mathrm{CP}_{2 \cdot \widehat{M}}$ & $\mathrm{~L}_{2 \cdot \widehat{M}}$ & $\mathrm{CP}_{2 \cdot \widehat{M}}$ & $\mathrm{~L}_{2 \cdot \widehat{M}}$ & $\mathrm{CP}_{2 \cdot \widehat{M}}$ & $\mathrm{~L}_{2 \cdot \widehat{M}}$ & $\mathrm{CP}_{2 \cdot \widehat{M}}$ & $\mathrm{~L}_{2 \cdot \widehat{M}}$ \\
\hline$\{1,2,3,4\}$ & .974 & 5.76 & 1.00 & 12.6 & 1.00 & 9.96 & .978 & 6.64 & .991 & 19.4 \\
\hline$\{1,2,4\}$ & .963 & 5.76 & 1.00 & 12.6 & .995 & 9.96 & .967 & 6.95 & .973 & 17.5 \\
\hline$\{1,2,4,5\}$ & .947 & 5.77 & 1.00 & 12.6 & 1.00 & 9.97 & .944 & 7.35 & .962 & 18.9 \\
\hline $\begin{array}{c}\text { Top Selected } \\
\text { Submodels }\end{array}$ & $\mathrm{CP}_{3 \cdot \widehat{M}}$ & $\mathrm{~L}_{3 \cdot \widehat{M}}$ & $\mathrm{CP}_{3 \cdot \widehat{M}}$ & $\mathrm{~L}_{3 \cdot \widehat{M}}$ & $\mathrm{CP}_{3 \cdot \widehat{M}}$ & $\mathrm{~L}_{3 \cdot \widehat{M}}$ & $\mathrm{CP}_{3 \cdot \widehat{M}}$ & $\mathrm{~L}_{3 \cdot \widehat{\boldsymbol{M}}}$ & $\mathrm{CP}_{3 \cdot \widehat{M}}$ & $\mathrm{~L}_{3 \cdot \widehat{M}}$ \\
\hline$\{1,3,4\}$ & .981 & 6.07 & 1.00 & 13.3 & 1.00 & 10.5 & .981 & 6.40 & .988 & 18.4 \\
\hline$\{1,2,3,4\}$ & .985 & 6.07 & 1.00 & 13.3 & 1.00 & 10.5 & .985 & 6.27 & .981 & 20.5 \\
\hline$\{1,3,4,5\}$ & .991 & 6.15 & 1.00 & 13.5 & 1.00 & 10.6 & .991 & 7.15 & .989 & 19.4 \\
\hline $\begin{array}{c}\text { Top Selected } \\
\text { Submodels }\end{array}$ & $\mathrm{CP}_{4 \cdot \widehat{M}}$ & $\mathrm{~L}_{4 \cdot \widehat{M}}$ & $\mathrm{CP}_{4 \cdot \widehat{M}}$ & $\mathrm{~L}_{4 \cdot \widehat{M}}$ & $\mathrm{CP}_{4 \cdot \widehat{M}}$ & $\mathrm{~L}_{4 \cdot \widehat{M}}$ & $\mathrm{CP}_{4 \cdot \widehat{M}}$ & $\mathrm{~L}_{4 \cdot \widehat{M}}$ & $\mathrm{CP}_{4 \cdot \widehat{M}}$ & $\mathrm{~L}_{4 \cdot \widehat{M}}$ \\
\hline$\{1,3,4\}$ & .979 & 6.34 & 1.00 & 13.9 & 1.00 & 11.0 & .979 & 6.33 & .970 & 20.9 \\
\hline$\{1,4\}$ & .971 & 5.88 & 1.00 & 12.9 & 1.00 & 10.2 & .971 & 5.87 & .957 & 12.0 \\
\hline$\{1,2,3,4\}$ & 1.00 & 6.45 & 1.00 & 14.1 & 1.00 & 11.1 & 1.00 & 6.89 & .981 & 22.3 \\
\hline $\begin{array}{l}\text { Top Selected } \\
\text { Submodels }\end{array}$ & $\mathrm{CP}_{5 \cdot \widehat{M}}$ & $\mathrm{~L}_{5 \cdot \widehat{M}}$ & $\mathrm{CP}_{5 \cdot \widehat{M}}$ & $\mathrm{~L}_{5 \cdot \widehat{M}}$ & $\mathrm{CP}_{5 \cdot \widehat{M}}$ & $\mathrm{~L}_{5 \cdot \widehat{M}}$ & $\mathrm{CP}_{5 \cdot \widehat{\boldsymbol{M}}}$ & $\mathrm{L}_{5 \cdot \widehat{M}}$ & $\mathrm{CP}_{5 \cdot \widehat{M}}$ & $\mathrm{~L}_{5 \cdot \widehat{M}}$ \\
\hline$\{1,3,4,5\}$ & .982 & 5.93 & 1.00 & 13.0 & 1.00 & 10.2 & .977 & 10.0 & .979 & 21.1 \\
\hline$\{1,4,5\}$ & .964 & 5.83 & 1.00 & 12.8 & 1.00 & 10.1 & .974 & 11.1 & .994 & 20.0 \\
\hline$\{1,2,4,5\}$ & .962 & 5.86 & 1.00 & 12.8 & 1.00 & 10.1 & .960 & 8.07 & .971 & 20.6 \\
\hline
\end{tabular}

$C P_{j \cdot \widehat{M}}$ and $L_{j \cdot \widehat{M}}, j=1$, 2, 3, 4 and 5, of post-selection confidence intervals based on Model II and $\boldsymbol{X}_{R l}$. 
S5.3. Model III $(n=40, p=14)$

\begin{tabular}{|c|c|c|c|c|c|c|c|c|c|c|}
\hline \multirow[b]{3}{*}{$\begin{array}{l}\text { Top Selected } \\
\text { Submodels }\end{array}$} & \multicolumn{10}{|c|}{ Post-selection Confidence Intervals } \\
\hline & \multicolumn{2}{|c|}{ Naïve } & \multicolumn{2}{|c|}{ Scheffé } & \multicolumn{2}{|c|}{ PoSI } & \multicolumn{2}{|c|}{ EPoSI1 } & \multicolumn{2}{|c|}{ EPoSI2 } \\
\hline & $\mathrm{CP}_{1 \cdot \widehat{M}}$ & $\mathrm{~L}_{1 \cdot \widehat{\boldsymbol{M}}}$ & $\mathrm{CP}_{1 \cdot \widehat{M}}$ & $\mathrm{~L}_{1 \cdot \widehat{M}}$ & $\mathrm{CP}_{1 \cdot \widehat{M}}$ & $\mathrm{~L}_{1 \cdot \widehat{M}}$ & $\mathrm{CP}_{1 \cdot \widehat{M}}$ & $\mathrm{~L}_{1 \cdot \widehat{\boldsymbol{M}}}$ & $\mathrm{CP}_{1 \cdot \widehat{M}}$ & $\mathrm{~L}_{1 \cdot \widehat{\boldsymbol{M}}}$ \\
\hline$\{1,2,3\}$ & .971 & 1.59 & 1.00 & 4.25 & 1.00 & 3.30 & .971 & 1.54 & .970 & 1.53 \\
\hline$\{1,2,3,13\}$ & .968 & 1.56 & 1.00 & 4.17 & 1.00 & 3.24 & .968 & 1.51 & .968 & 1.82 \\
\hline$\{1,2,3,8\}$ & .938 & 1.54 & 1.00 & 4.12 & 1.00 & 3.20 & .938 & 1.49 & .924 & 2.35 \\
\hline $\begin{array}{l}\text { Top Selected } \\
\text { Submodels }\end{array}$ & $\mathrm{CP}_{2 \cdot \widehat{M}}$ & $\mathrm{~L}_{2 \cdot \widehat{M}}$ & $\mathrm{CP}_{2 \cdot \widehat{M}}$ & $\mathrm{~L}_{2 \cdot \widehat{M}}$ & $\mathrm{CP}_{2 \cdot \widehat{M}}$ & $\mathrm{~L}_{2 \cdot \widehat{M}}$ & $\mathrm{CP}_{2 \cdot \widehat{M}}$ & $\mathrm{~L}_{2 \cdot \widehat{M}}$ & $\mathrm{CP}_{2 \cdot \widehat{M}}$ & $\mathrm{~L}_{2 \cdot \widehat{M}}$ \\
\hline 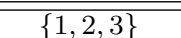 & .941 & 1.53 & 1.00 & 4.10 & 1.00 & 3.18 & .926 & 1.48 & .924 & 1.48 \\
\hline$\{1,2,3,13\}$ & .958 & 1.64 & 1.00 & 4.38 & 1.00 & 3.40 & .947 & 1.59 & .989 & 3.22 \\
\hline$\{1,2,3,8\}$ & .963 & 1.55 & 1.00 & 4.13 & .988 & 3.21 & .938 & 1.50 & .936 & 2.54 \\
\hline $\begin{array}{l}\text { Top Selected } \\
\text { Submodels }\end{array}$ & $\mathrm{CP}_{3 \cdot \widehat{M}}$ & $\mathrm{~L}_{3 \cdot \widehat{M}}$ & $\mathrm{CP}_{3 \cdot \widehat{M}}$ & $\mathrm{~L}_{3 \cdot \widehat{\boldsymbol{M}}}$ & $\mathrm{CP}_{3 \cdot \widehat{M}}$ & $\mathrm{~L}_{3 \cdot \widehat{M}}$ & $\mathrm{CP}_{3 \cdot \widehat{M}}$ & $\mathrm{~L}_{3 \cdot \widehat{M}}$ & $\mathrm{CP}_{3 \cdot \widehat{M}}$ & $\mathrm{~L}_{3 \cdot \widehat{M}}$ \\
\hline$\{1,2,3\}$ & .971 & 1.75 & 1.00 & 4.68 & 1.00 & 3.63 & .963 & 1.70 & .970 & 1.69 \\
\hline$\{1,2,3,13\}$ & .958 & 2.05 & 1.00 & 5.47 & 1.00 & 4.24 & .947 & 1.98 & .968 & 4.80 \\
\hline$\{1,2,3,8\}$ & .950 & 1.80 & 1.00 & 4.80 & 1.00 & 3.72 & .913 & 1.74 & .962 & 3.09 \\
\hline
\end{tabular}

$C P_{j \cdot \widehat{M}}$ and $L_{j \cdot \widehat{M}}, j=1,2$ and 3, of post-selection confidence intervals based on Model III and $\boldsymbol{X}_{E q}$.

\begin{tabular}{|c|c|c|c|c|c|c|c|c|c|c|}
\hline \multirow[b]{3}{*}{$\begin{array}{l}\text { Top Selected } \\
\text { Submodels }\end{array}$} & \multicolumn{10}{|c|}{ Post-selection Confidence Intervals } \\
\hline & \multicolumn{2}{|c|}{ Naïve } & \multicolumn{2}{|c|}{ Scheffé } & \multicolumn{2}{|c|}{ PoSI } & \multicolumn{2}{|c|}{ EPoSI1 } & \multicolumn{2}{|c|}{ EPoSI2 } \\
\hline & $\mathrm{CP}_{1 \cdot \widehat{M}}$ & $\mathrm{~L}_{1 \cdot \widehat{M}}$ & $\mathrm{CP}_{1 \cdot \widehat{M}}$ & $\mathrm{~L}_{1 \cdot \widehat{M}}$ & $\mathrm{CP}_{1 \cdot \widehat{M}}$ & $\mathrm{~L}_{1 \cdot \widehat{M}}$ & $\mathrm{CP}_{1 \cdot \widehat{M}}$ & $\mathrm{~L}_{1 \cdot \widehat{M}}$ & $\mathrm{CP}_{1 \cdot \widehat{M}}$ & $\mathrm{~L}_{1 \cdot \widehat{M}}$ \\
\hline$\{1,2,3\}$ & .950 & 1.90 & 1.00 & 5.07 & 1.00 & 3.97 & .943 & 1.83 & .943 & 1.83 \\
\hline$\{1,2,3,4\}$ & .930 & 1.92 & 1.00 & 5.13 & 1.00 & 4.01 & .925 & 2.06 & .940 & 2.85 \\
\hline$\{1,2,3,14\}$ & .919 & 1.96 & 1.00 & 5.23 & 1.00 & 4.09 & .910 & 1.89 & .940 & 3.66 \\
\hline $\begin{array}{l}\text { Top Selected } \\
\text { Submodels }\end{array}$ & $\mathrm{CP}_{2 \cdot \widehat{M}}$ & $\mathrm{~L}_{2 \cdot \widehat{M}}$ & $\mathrm{CP}_{2 \cdot \widehat{M}}$ & $\mathrm{~L}_{2 \cdot \widehat{M}}$ & $\mathrm{CP}_{2 \cdot \widehat{M}}$ & $\mathrm{~L}_{2 \cdot \widehat{M}}$ & $\mathrm{CP}_{2 \cdot \widehat{M}}$ & $\mathrm{~L}_{2 \cdot \widehat{M}}$ & $\mathrm{CP}_{2 \cdot \widehat{M}}$ & $\mathrm{~L}_{2 \cdot \widehat{M}}$ \\
\hline$\{1,2,3\}$ & .950 & 2.75 & 1.00 & 7.34 & 1.00 & 5.74 & .944 & 2.65 & .944 & 2.66 \\
\hline$\{1,2,3,4\}$ & .958 & 2.74 & 1.00 & 7.31 & 1.00 & 5.72 & .947 & 2.71 & .946 & 2.92 \\
\hline$\{1,2,3,13\}$ & .942 & 2.78 & 1.00 & 7.41 & 1.00 & 5.80 & .933 & 2.68 & .947 & 4.30 \\
\hline $\begin{array}{l}\text { Top Selected } \\
\text { Submodels }\end{array}$ & $\mathrm{CP}_{3 \cdot \widehat{M}}$ & $\mathrm{~L}_{3 \cdot \widehat{M}}$ & $\mathrm{CP}_{3 \cdot \widehat{M}}$ & $\mathrm{~L}_{3 \cdot \widehat{M}}$ & $\mathrm{CP}_{3 \cdot \widehat{M}}$ & $\mathrm{~L}_{3 \cdot \widehat{M}}$ & $\mathrm{CP}_{3 \cdot \widehat{M}}$ & $\mathrm{~L}_{3 \cdot \widehat{M}}$ & $\mathrm{CP}_{3 \cdot \widehat{M}}$ & $\mathrm{~L}_{3 \cdot \widehat{M}}$ \\
\hline$\{1,2,3\}$ & .951 & 2.64 & 1.00 & 7.06 & 1.00 & 5.53 & .942 & 2.56 & .942 & 2.56 \\
\hline$\{1,2,3,4\}$ & .928 & 3.10 & 1.00 & 8.27 & 1.00 & 6.47 & .919 & 3.15 & .957 & 7.16 \\
\hline$\{1,2,3,14\}$ & .947 & 2.71 & 1.00 & 7.24 & 1.00 & 5.67 & .940 & 2.62 & .952 & 4.94 \\
\hline
\end{tabular}

$C P_{j \cdot \widehat{M}}$ and $L_{j \cdot \widehat{M}}, j=1,2$ and 3, of post-selection confidence intervals based on Model III and $\boldsymbol{X}_{T p}$. 


\begin{tabular}{|c|c|c|c|c|c|c|c|c|c|c|}
\hline & \multicolumn{10}{|c|}{ Post-selection Confidence Intervals } \\
\hline & \multicolumn{2}{|c|}{ Naïve } & \multicolumn{2}{|c|}{ Scheffé } & \multicolumn{2}{|c|}{ PoSI } & \multicolumn{2}{|c|}{ EPoSI1 } & \multicolumn{2}{|c|}{ EPoSI2 } \\
\hline $\begin{array}{l}\text { Top Selected } \\
\text { Submodels }\end{array}$ & $\mathrm{CP}_{1 \cdot \widehat{M}}$ & $\mathrm{~L}_{1 \cdot \widehat{M}}$ & $\mathrm{CP}_{1 \cdot \widehat{M}}$ & $\mathrm{~L}_{1 \cdot \widehat{M}}$ & $\mathrm{CP}_{1 \cdot \widehat{M}}$ & $\mathrm{~L}_{1 \cdot \widehat{M}}$ & $\mathrm{CP}_{1 \cdot \widehat{M}}$ & $\mathrm{~L}_{1 \cdot \widehat{M}}$ & $\mathrm{CP}_{1 \cdot \widehat{M}}$ & $\mathrm{~L}_{1 \cdot \widehat{M}}$ \\
\hline$\{1,2,3,4\}$ & .948 & 1.14 & 1.00 & 3.04 & 1.00 & 2.26 & .939 & 1.10 & .939 & 1.12 \\
\hline$\{1,2,3\}$ & .944 & 1.14 & 1.00 & 3.04 & 1.00 & 2.26 & .939 & 1.10 & .939 & 1.10 \\
\hline$\{1,2,3,4,14\}$ & .942 & 1.15 & 1.00 & 3.07 & 1.00 & 2.29 & .923 & 1.11 & .928 & 2.23 \\
\hline $\begin{array}{c}\text { Top Selected } \\
\text { Submodels }\end{array}$ & $\mathrm{CP}_{2 \cdot \widehat{M}}$ & $\mathrm{~L}_{2 \cdot \widehat{M}}$ & $\mathrm{CP}_{2 \cdot \widehat{M}}$ & $\mathrm{~L}_{2 \cdot \widehat{M}}$ & $\mathrm{CP}_{2 \cdot \widehat{M}}$ & $\mathrm{~L}_{2 \cdot \widehat{M}}$ & $\mathrm{CP}_{2 \cdot \widehat{M}}$ & $\mathrm{~L}_{2 \cdot \widehat{M}}$ & $\mathrm{CP}_{2 \cdot \widehat{M}}$ & $\mathrm{~L}_{2 \cdot \widehat{M}}$ \\
\hline$\{1,2,3,4\}$ & .947 & 1.40 & 1.00 & 3.72 & .999 & 2.77 & .940 & 1.35 & .946 & 2.08 \\
\hline$\{1,2,3\}$ & .950 & 1.33 & 1.00 & 3.55 & 1.00 & 2.64 & .942 & 1.28 & .942 & 1.29 \\
\hline$\{1,2,3,4,14\}$ & .956 & 1.39 & 1.00 & 3.72 & 1.00 & 2.77 & .945 & 1.34 & .964 & 2.64 \\
\hline $\begin{array}{l}\text { Top Selected } \\
\text { Submodels }\end{array}$ & $\mathrm{CP}_{3 \cdot \widehat{M}}$ & $\mathrm{~L}_{3 \cdot \widehat{M}}$ & $\mathrm{CP}_{3 \cdot \widehat{M}}$ & $\mathrm{~L}_{3 \cdot \widehat{M}}$ & $\mathrm{CP}_{3 \cdot \widehat{M}}$ & $\mathrm{~L}_{3 \cdot \widehat{M}}$ & $\mathrm{CP}_{3 \cdot \widehat{M}}$ & $\mathrm{~L}_{3 \cdot \widehat{M}}$ & $\mathrm{CP}_{3 \cdot \widehat{M}}$ & $\mathrm{~L}_{3 \cdot \widehat{M}}$ \\
\hline$\{1,2,3,4\}$ & .952 & 1.34 & 1.00 & 3.59 & 1.00 & 2.67 & .948 & 1.68 & .954 & 2.51 \\
\hline$\{1,2,3\}$ & .947 & 1.11 & 1.00 & 2.97 & 1.00 & 2.21 & .941 & 1.07 & .941 & 1.07 \\
\hline$\{1,2,3,4,13\}$ & .934 & 1.36 & 1.00 & 3.61 & 1.00 & 2.69 & .928 & 1.31 & .977 & 3.16 \\
\hline
\end{tabular}

$C P_{j \cdot \widehat{M}}$ and $L_{j \cdot \widehat{M}}, j=1,2$ and 3, of post-selection confidence intervals based on Model III and $\boldsymbol{X}_{E p}$.

\begin{tabular}{|c|c|c|c|c|c|c|c|c|c|c|}
\hline & \multicolumn{10}{|c|}{ Post-selection Confidence Intervals } \\
\hline & \multicolumn{2}{|c|}{ Naïve } & \multicolumn{2}{|c|}{ Scheffé } & \multicolumn{2}{|c|}{ PoSI } & \multicolumn{2}{|c|}{ EPoSI1 } & \multicolumn{2}{|c|}{ EPoSI2 } \\
\hline $\begin{array}{l}\text { Top Selected } \\
\text { Submodels }\end{array}$ & $\mathrm{CP}_{1 \cdot \widehat{M}}$ & $\mathrm{~L}_{1 \cdot \widehat{M}}$ & $\mathrm{CP}_{1 \cdot \widehat{M}}$ & $\mathrm{~L}_{1 \cdot \widehat{\boldsymbol{M}}}$ & $\mathrm{CP}_{1 \cdot \widehat{M}}$ & $\mathrm{~L}_{1 \cdot \widehat{M}}$ & $\mathrm{CP}_{1 \cdot \widehat{M}}$ & $\mathrm{~L}_{1 \cdot \widehat{M}}$ & $\mathrm{CP}_{1 \cdot \widehat{M}}$ & $\mathrm{~L}_{1 \cdot \widehat{M}}$ \\
\hline$\{1,2,3\}$ & .947 & 4.61 & 1.00 & 12.3 & 1.00 & 9.45 & .939 & 4.46 & .939 & 4.53 \\
\hline$\{1,2,3,4\}$ & .916 & 4.93 & 1.00 & 13.2 & 1.00 & 10.1 & .911 & 4.78 & 1.00 & 5.20 \\
\hline$\{1,2,3,5\}$ & .907 & 4.91 & 1.00 & 13.1 & .992 & 10.0 & .890 & 4.74 & 1.00 & 5.23 \\
\hline $\begin{array}{l}\text { Top Selected } \\
\text { Submodels }\end{array}$ & $\mathrm{CP}_{2 \cdot \widehat{M}}$ & $\mathrm{~L}_{2 \cdot \widehat{M}}$ & $\mathrm{CP}_{2 \cdot \widehat{M}}$ & $\mathrm{~L}_{2 \cdot \widehat{M}}$ & $\mathrm{CP}_{2 \cdot \widehat{M}}$ & $\mathrm{~L}_{2 \cdot \widehat{M}}$ & $\mathrm{CP}_{2 \cdot \widehat{M}}$ & $\mathrm{~L}_{2 \cdot \widehat{M}}$ & $\mathrm{CP}_{2 \cdot \widehat{M}}$ & $\mathrm{~L}_{2 \cdot \widehat{M}}$ \\
\hline$\{1,2,3\}$ & .959 & 4.61 & 1.00 & 12.3 & .999 & 9.45 & .952 & 4.46 & .939 & 4.51 \\
\hline$\{1,2,3,4\}$ & .832 & 4.93 & 1.00 & 13.2 & 1.00 & 10.1 & .812 & 4.76 & .909 & 5.44 \\
\hline$\{1,2,3,5\}$ & .865 & 4.91 & 1.00 & 13.1 & 1.00 & 10.0 & .848 & 4.74 & 1.00 & 5.60 \\
\hline $\begin{array}{l}\text { Top Selected } \\
\text { Submodels }\end{array}$ & $\mathrm{CP}_{3 \cdot \widehat{M}}$ & $\mathrm{~L}_{3 \cdot \widehat{M}}$ & $\mathrm{CP}_{3 \cdot \widehat{M}}$ & $\mathrm{~L}_{3 \cdot \widehat{M}}$ & $\mathrm{CP}_{3 \cdot \widehat{M}}$ & $\mathrm{~L}_{3 \cdot \widehat{M}}$ & $\mathrm{CP}_{3 \cdot \widehat{M}}$ & $\mathrm{~L}_{3 \cdot \widehat{M}}$ & $\mathrm{CP}_{3 \cdot \widehat{M}}$ & $\mathrm{~L}_{3 \cdot \widehat{M}}$ \\
\hline$\{1,2,3\}$ & .959 & 4.61 & 1.00 & 12.3 & 1.00 & 9.45 & .952 & 4.46 & .948 & 4.50 \\
\hline$\{1,2,3,4\}$ & .924 & 4.93 & 1.00 & 13.2 & 1.00 & 10.1 & .911 & 4.78 & .909 & 5.44 \\
\hline$\{1,2,3,5\}$ & .873 & 4.91 & 1.00 & 13.1 & 1.00 & 10.0 & .865 & 4.74 & .750 & 5.27 \\
\hline
\end{tabular}

$C P_{j \cdot \widehat{M}}$ and $L_{j \cdot \widehat{M}}, j=1,2$ and 3, of post-selection confidence intervals based on Model III and $\boldsymbol{X}_{E c}$. 


\begin{tabular}{|c|c|c|c|c|c|c|c|c|c|c|}
\hline \multirow[b]{3}{*}{$\begin{array}{l}\text { Top Selected } \\
\text { Submodels }\end{array}$} & \multicolumn{10}{|c|}{ Post-selection Confidence Intervals } \\
\hline & \multicolumn{2}{|c|}{ Naïve } & \multicolumn{2}{|c|}{ Scheffé } & \multicolumn{2}{|c|}{ PoSI } & \multicolumn{2}{|c|}{ EPoSI1 } & \multicolumn{2}{|c|}{ EPoSI2 } \\
\hline & $\mathrm{CP}_{1 \cdot \widehat{M}}$ & $\mathrm{~L}_{1 \cdot \widehat{M}}$ & $\mathrm{CP}_{1 \cdot \widehat{M}}$ & $\mathrm{~L}_{1 \cdot \widehat{M}}$ & $\mathrm{CP}_{1 \cdot \widehat{M}}$ & $\mathrm{~L}_{1 \cdot \widehat{M}}$ & $\mathrm{CP}_{1 \cdot \widehat{M}}$ & $\mathrm{~L}_{1 \cdot \widehat{M}}$ & $\mathrm{CP}_{1 \cdot \widehat{M}}$ & $\mathrm{~L}_{1 \cdot \widehat{M}}$ \\
\hline$\{1,2,3\}$ & .976 & 5.73 & 1.00 & 12.5 & 1.00 & 9.86 & .975 & 6.03 & .961 & 11.8 \\
\hline$\{1,2,3,4\}$ & .992 & 5.96 & 1.00 & 13.0 & 1.00 & 10.3 & .990 & 6.91 & .971 & 17.9 \\
\hline$\{1,2,3,10\}$ & .994 & 5.89 & 1.00 & 12.9 & 1.00 & 10.1 & .987 & 7.75 & .969 & 17.5 \\
\hline $\begin{array}{l}\text { Top Selected } \\
\text { Submodels }\end{array}$ & $\mathrm{CP}_{2 \cdot \widehat{M}}$ & $\mathrm{~L}_{2 \cdot \widehat{M}}$ & $\mathrm{CP}_{2 \cdot \widehat{M}}$ & $\mathrm{~L}_{2 \cdot \widehat{M}}$ & $\mathrm{CP}_{2 \cdot \widehat{M}}$ & $\mathrm{~L}_{2 \cdot \widehat{M}}$ & $\mathrm{CP}_{2 \cdot \widehat{M}}$ & $\mathrm{~L}_{2 \cdot \widehat{M}}$ & $\mathrm{CP}_{2 \cdot \widehat{M}}$ & $\mathrm{~L}_{2 \cdot \widehat{M}}$ \\
\hline$\{1,2,3\}$ & .977 & 5.65 & 1.00 & 12.4 & 1.00 & 9.73 & .977 & 5.66 & .969 & 10.6 \\
\hline$\{1,2,3,4\}$ & .986 & 5.77 & 1.00 & 12.6 & 1.00 & 9.94 & .988 & 6.22 & .969 & 14.6 \\
\hline$\{1,2,3,10\}$ & .994 & 5.73 & 1.00 & 12.5 & 1.00 & 9.86 & .991 & 5.92 & .977 & 14.6 \\
\hline $\begin{array}{c}\text { Top Selected } \\
\text { Submodels }\end{array}$ & $\mathrm{CP}_{3 \cdot \widehat{M}}$ & $\mathrm{~L}_{3 \cdot \widehat{M}}$ & $\mathrm{CP}_{3 \cdot \widehat{M}}$ & $\mathrm{~L}_{3 \cdot \widehat{M}}$ & $\mathrm{CP}_{3 \cdot \widehat{M}}$ & $\mathrm{~L}_{3 \cdot \widehat{M}}$ & $\mathrm{CP}_{3 \cdot \widehat{M}}$ & $\mathrm{~L}_{3 \cdot \widehat{M}}$ & $\mathrm{CP}_{3 \cdot \widehat{M}}$ & $\mathrm{~L}_{3 \cdot \widehat{M}}$ \\
\hline$\{1,2,3\}$ & .974 & 5.66 & 1.00 & 12.4 & 1.00 & 9.75 & .974 & 5.70 & .963 & 11.1 \\
\hline$\{1,2,3,4\}$ & .993 & 6.08 & 1.00 & 13.3 & 1.00 & 10.5 & .992 & 6.80 & .976 & 20.0 \\
\hline$\{1,2,3,10\}$ & .998 & 6.05 & 1.00 & 13.3 & 1.00 & 10.4 & .998 & 6.04 & .990 & 20.0 \\
\hline
\end{tabular}

$C P_{j \cdot \widehat{M}}$ and $L_{j \cdot \widehat{M}}, j=1,2$ and 3, of post-selection confidence intervals based on Model III and $\boldsymbol{X}_{R l}$. 
S5.4. $\quad$ Model IV $(n=40, p=14)$

\begin{tabular}{|c|c|c|c|c|c|c|c|c|c|c|}
\hline & \multicolumn{10}{|c|}{ Post-selection Confidence Intervals } \\
\hline & \multicolumn{2}{|c|}{ Naïve } & \multicolumn{2}{|c|}{ Scheffé } & \multicolumn{2}{|c|}{ PoSI } & \multicolumn{2}{|c|}{ EPoSI1 } & \multicolumn{2}{|c|}{ EPoSI2 } \\
\hline $\begin{array}{l}\text { Top Selected } \\
\text { Submodels }\end{array}$ & $\mathrm{CP}_{1 \cdot \widehat{M}}$ & $\mathrm{~L}_{1 \cdot \widehat{M}}$ & $\mathrm{CP}_{1 \cdot \widehat{M}}$ & $\mathrm{~L}_{1 \cdot \widehat{M}}$ & $\mathrm{CP}_{1 \cdot \widehat{M}}$ & $\mathrm{~L}_{1 \cdot \widehat{M}}$ & $\mathrm{CP}_{1 \cdot \widehat{M}}$ & $\mathrm{~L}_{1 \cdot \widehat{M}}$ & $\mathrm{CP}_{1 \cdot \widehat{M}}$ & $\mathrm{~L}_{1 \cdot \widehat{M}}$ \\
\hline$\{1,2,3,4,5\}$ & .947 & 1.88 & 1.00 & 5.01 & 1.00 & 3.83 & .943 & 1.81 & .944 & 1.81 \\
\hline$\{1,2,3,4,5,7\}$ & .944 & 1.85 & 1.00 & 4.92 & 1.00 & 3.76 & .939 & 1.78 & .948 & 1.82 \\
\hline$\{1,2,3,4,5,13\}$ & .939 & 1.89 & 1.00 & 5.03 & 1.00 & 3.84 & .928 & 1.82 & .943 & 2.64 \\
\hline $\begin{array}{l}\text { Top Selected } \\
\text { Submodels }\end{array}$ & $\mathrm{CP}_{2 \cdot \widehat{M}}$ & $\mathrm{~L}_{2 \cdot \widehat{M}}$ & $\mathrm{CP}_{2 \cdot \widehat{M}}$ & $\mathrm{~L}_{2 \cdot \widehat{M}}$ & $\mathrm{CP}_{2 \cdot \widehat{M}}$ & $\mathrm{~L}_{2 \cdot \widehat{M}}$ & $\mathrm{CP}_{2 \cdot \widehat{M}}$ & $\mathrm{~L}_{2 \cdot \widehat{M}}$ & $\mathrm{CP}_{2 \cdot \widehat{M}}$ & $\mathrm{~L}_{2 \cdot \widehat{M}}$ \\
\hline$\{1,2,3,4,5\}$ & .959 & 2.46 & 1.00 & 6.56 & .996 & 5.01 & .951 & 2.37 & .949 & 2.37 \\
\hline$\{1,2,3,4,5,7\}$ & .948 & 2.47 & 1.00 & 6.59 & .996 & 5.03 & .940 & 2.38 & .934 & 3.10 \\
\hline$\{1,2,3,4,5,13\}$ & .967 & 2.44 & 1.00 & 6.49 & 1.00 & 4.96 & .967 & 2.35 & .960 & 2.74 \\
\hline $\begin{array}{l}\text { Top Selected } \\
\text { Submodels }\end{array}$ & $\mathrm{CP}_{3 \cdot \widehat{M}}$ & $\mathrm{~L}_{3 \cdot \widehat{M}}$ & $\mathrm{CP}_{3 \cdot \widehat{M}}$ & $\mathrm{~L}_{3 \cdot \widehat{M}}$ & $\mathrm{CP}_{3 \cdot \widehat{M}}$ & $\mathrm{~L}_{3 \cdot \widehat{M}}$ & $\mathrm{CP}_{3 \cdot \widehat{M}}$ & $\mathrm{~L}_{3 \cdot \widehat{M}}$ & $\mathrm{CP}_{3 \cdot \widehat{M}}$ & $\mathrm{~L}_{3 \cdot \widehat{M}}$ \\
\hline$\{1,2,3,4,5\}$ & .959 & 2.17 & 1.00 & 5.79 & 1.00 & 4.43 & .955 & 2.10 & .953 & 2.09 \\
\hline$\{1,2,3,4,5,7\}$ & .944 & 2.19 & 1.00 & 5.84 & 1.00 & 4.46 & .944 & 2.11 & .958 & 2.84 \\
\hline$\{1,2,3,4,5,13\}$ & .923 & 2.15 & 1.00 & 5.72 & 1.00 & 4.37 & .912 & 2.07 & .915 & 2.28 \\
\hline $\begin{array}{c}\text { Top Selected } \\
\text { Submodels }\end{array}$ & $\mathrm{CP}_{4 \cdot \widehat{M}}$ & $\mathrm{~L}_{4 \cdot \widehat{M}}$ & $\mathrm{CP}_{4 \cdot \widehat{M}}$ & $\mathrm{~L}_{4 \cdot \widehat{M}}$ & $\mathrm{CP}_{4 \cdot \widehat{M}}$ & $\mathrm{~L}_{4 \cdot \widehat{M}}$ & $\mathrm{CP}_{4 \cdot \widehat{M}}$ & $\mathrm{~L}_{4 \cdot \widehat{M}}$ & $\mathrm{CP}_{4 \cdot \widehat{M}}$ & $\mathrm{~L}_{4 \cdot \widehat{M}}$ \\
\hline$\{1,2,3,4,5\}$ & .943 & 2.17 & 1.00 & 5.78 & 1.00 & 4.42 & .939 & 2.09 & .940 & 2.09 \\
\hline$\{1,2,3,4,5,7\}$ & .940 & 2.16 & 1.00 & 5.76 & 1.00 & 4.40 & .931 & 2.09 & .948 & 2.57 \\
\hline$\{1,2,3,4,5,13\}$ & .945 & 2.25 & 1.00 & 5.99 & 1.00 & 4.57 & .945 & 2.17 & .943 & 3.70 \\
\hline $\begin{array}{l}\text { Top Selected } \\
\text { Submodels }\end{array}$ & $\mathrm{CP}_{5 \cdot \widehat{M}}$ & $\mathrm{~L}_{5 \cdot \widehat{M}}$ & $\mathrm{CP}_{5 \cdot \widehat{M}}$ & $\mathrm{~L}_{5 \cdot \widehat{M}}$ & $\mathrm{CP}_{5 \cdot \widehat{M}}$ & $\mathrm{~L}_{5 \cdot \widehat{M}}$ & $\mathrm{CP}_{5 \cdot \widehat{M}}$ & $\mathrm{~L}_{5 \cdot \widehat{M}}$ & $\mathrm{CP}_{5 \cdot \widehat{M}}$ & $\mathrm{~L}_{5 \cdot \widehat{M}}$ \\
\hline$\{1,2,3,4,5\}$ & .967 & 1.96 & 1.00 & 5.23 & 1.00 & 4.00 & .951 & 1.89 & .949 & $\overline{1.89}$ \\
\hline$\{1,2,3,4,5,7\}$ & .961 & 2.03 & 1.00 & 5.40 & 1.00 & 4.12 & .944 & 1.95 & .958 & 2.97 \\
\hline$\{1,2,3,4,5,13\}$ & .945 & 2.02 & 1.00 & 5.38 & 1.00 & 4.11 & .934 & 1.95 & .943 & 3.34 \\
\hline
\end{tabular}

$C P_{j \cdot \widehat{M}}$ and $L_{j \cdot \widehat{M}}, j=1,2,3,4$ and 5, of post-selection confidence intervals based on Model

$I V$ and $\boldsymbol{X}_{E q}$. 


\begin{tabular}{|c|c|c|c|c|c|c|c|c|c|c|}
\hline & \multicolumn{10}{|c|}{ Post-selection Confidence Intervals } \\
\hline & \multicolumn{2}{|c|}{ Naïve } & \multicolumn{2}{|c|}{ Scheffé } & \multicolumn{2}{|c|}{ PoSI } & \multicolumn{2}{|c|}{ EPoSI1 } & \multicolumn{2}{|c|}{ EPoSI2 } \\
\hline $\begin{array}{c}\text { Top Selected } \\
\text { Submodels }\end{array}$ & $\mathrm{CP}_{1 \cdot \widehat{M}}$ & $\mathrm{~L}_{1 \cdot \widehat{M}}$ & $\mathrm{CP}_{1 \cdot \widehat{M}}$ & $\mathrm{~L}_{1 \cdot \widehat{M}}$ & $\mathrm{CP}_{1 \cdot \widehat{M}}$ & $\mathrm{~L}_{1 \cdot \widehat{M}}$ & $\mathrm{CP}_{1 \cdot \widehat{M}}$ & $\mathrm{~L}_{1 \cdot \widehat{M}}$ & $\mathrm{CP}_{1 \cdot \widehat{M}}$ & $\mathrm{~L}_{1 \cdot \widehat{M}}$ \\
\hline$\{1,2,3,4,5\}$ & .940 & 2.38 & 1.00 & 6.34 & .999 & 4.90 & .931 & 2.29 & .931 & 2.29 \\
\hline$\{1,2,3,4,5,6\}$ & .943 & 2.43 & 1.00 & 6.47 & 1.00 & 5.01 & .938 & 2.49 & .949 & 3.66 \\
\hline$\{1,2,3,4,5,7\}$ & .944 & 2.44 & 1.00 & 6.49 & 1.00 & 5.02 & .953 & 3.51 & .958 & 3.88 \\
\hline $\begin{array}{l}\text { Top Selected } \\
\text { Submodels }\end{array}$ & $\mathrm{CP}_{2 \cdot \widehat{M}}$ & $\mathrm{~L}_{2 \cdot \widehat{M}}$ & $\mathrm{CP}_{2 \cdot \widehat{M}}$ & $\mathrm{~L}_{2 \cdot \widehat{M}}$ & $\mathrm{CP}_{2 \cdot \widehat{M}}$ & $\mathrm{~L}_{2 \cdot \widehat{M}}$ & $\mathrm{CP}_{2 \cdot \widehat{M}}$ & $\mathrm{~L}_{2 \cdot \widehat{M}}$ & $\mathrm{CP}_{2 \cdot \widehat{M}}$ & $\mathrm{~L}_{2 \cdot \widehat{M}}$ \\
\hline$\{1,2,3,4,5\}$ & .949 & 2.96 & 1.00 & 7.90 & 1.00 & 6.12 & .938 & 2.86 & .938 & 2.86 \\
\hline$\{1,2,3,4,5,6\}$ & .951 & 3.15 & 1.00 & 8.40 & 1.00 & 6.50 & .948 & 3.22 & .961 & 6.24 \\
\hline$\{1,2,3,4,5,7\}$ & .935 & 3.26 & 1.00 & 8.69 & .999 & 6.72 & .956 & 5.88 & .972 & 7.16 \\
\hline $\begin{array}{c}\text { Top Selected } \\
\text { Submodels }\end{array}$ & $\mathrm{CP}_{3 \cdot \widehat{M}}$ & $\mathrm{~L}_{3 \cdot \widehat{M}}$ & $\mathrm{CP}_{3 \cdot \widehat{M}}$ & $\mathrm{~L}_{3 \cdot \widehat{M}}$ & $\mathrm{CP}_{3 \cdot \widehat{M}}$ & $\mathrm{~L}_{3 \cdot \widehat{M}}$ & $\mathrm{CP}_{3 \cdot \widehat{M}}$ & $\mathrm{~L}_{3 \cdot \widehat{\boldsymbol{M}}}$ & $\mathrm{CP}_{3 \cdot \widehat{M}}$ & $\mathrm{~L}_{3 \cdot \widehat{M}}$ \\
\hline$\{1,2,3,4,5\}$ & .945 & 3.24 & 1.00 & 8.65 & 1.00 & 6.69 & .937 & 3.13 & .937 & 3.13 \\
\hline$\{1,2,3,4,5,6\}$ & .950 & 3.26 & 1.00 & 8.68 & 1.00 & 6.72 & .933 & 3.15 & .932 & 3.21 \\
\hline$\{1,2,3,4,5,7\}$ & .926 & 3.37 & 1.00 & 8.99 & 1.00 & 6.95 & .914 & 3.27 & .941 & 5.87 \\
\hline $\begin{array}{c}\text { Top Selected } \\
\text { Submodels }\end{array}$ & $\mathrm{CP}_{4 \cdot \widehat{M}}$ & $\mathrm{~L}_{4 \cdot \widehat{M}}$ & $\mathrm{CP}_{4 \cdot \widehat{M}}$ & $\mathrm{~L}_{4 \cdot \widehat{M}}$ & $\mathrm{CP}_{4 \cdot \widehat{M}}$ & $\mathrm{~L}_{4 \cdot \widehat{M}}$ & $\mathrm{CP}_{4 \cdot \widehat{M}}$ & $\mathrm{~L}_{4 \cdot \widehat{M}}$ & $\mathrm{CP}_{4 \cdot \widehat{M}}$ & $\mathrm{~L}_{4 \cdot \widehat{M}}$ \\
\hline$\{1,2,3,4,5\}$ & .942 & 3.08 & 1.00 & 8.20 & 1.00 & 6.35 & .934 & 2.97 & .934 & 2.97 \\
\hline$\{1,2,3,4,5,6\}$ & .946 & 3.11 & 1.00 & 8.30 & 1.00 & 6.42 & .937 & 3.00 & .947 & 4.22 \\
\hline$\{1,2,3,4,5,7\}$ & .954 & 3.10 & 1.00 & 8.25 & 1.00 & 6.38 & .946 & 3.41 & .948 & 3.46 \\
\hline $\begin{array}{c}\text { Top Selected } \\
\text { Submodels }\end{array}$ & $\mathrm{CP}_{5 \cdot \widehat{M}}$ & $\mathrm{~L}_{5 \cdot \widehat{M}}$ & $\mathrm{CP}_{5 \cdot \widehat{M}}$ & $\mathrm{~L}_{5 \cdot \widehat{M}}$ & $\mathrm{CP}_{5 \cdot \widehat{M}}$ & $\mathrm{~L}_{5 \cdot \widehat{M}}$ & $\mathrm{CP}_{5 \cdot \widehat{M}}$ & $\mathrm{~L}_{5 \cdot \widehat{M}}$ & $\mathrm{CP}_{5 \cdot \widehat{M}}$ & $\mathrm{~L}_{5 \cdot \widehat{M}}$ \\
\hline$\{1,2,3,4,5\}$ & .948 & 2.05 & 1.00 & 5.46 & .999 & 4.22 & .940 & 1.97 & .940 & 1.97 \\
\hline$\{1,2,3,4,5,6\}$ & .897 & 3.11 & 1.00 & 8.29 & 1.00 & 6.41 & .883 & 3.03 & .960 & 8.81 \\
\hline$\{1,2,3,4,5,7\}$ & .928 & 2.33 & 1.00 & 6.20 & 1.00 & 4.80 & .917 & 2.24 & .953 & 5.45 \\
\hline
\end{tabular}

$C P_{j \cdot \widehat{M}}$ and $L_{j \cdot \widehat{M}}, j=1,2,3,4$ and 5, of post-selection confidence intervals based on Model IV and $\boldsymbol{X}_{T p}$. 


\begin{tabular}{|c|c|c|c|c|c|c|c|c|c|c|}
\hline \multirow[b]{3}{*}{$\begin{array}{l}\text { Top Selected } \\
\text { Submodels }\end{array}$} & \multicolumn{10}{|c|}{ Post-selection Confidence Intervals } \\
\hline & \multicolumn{2}{|c|}{ Naïve } & \multicolumn{2}{|c|}{ Scheffé } & \multicolumn{2}{|c|}{ PoSI } & \multicolumn{2}{|c|}{ EPoSI1 } & \multicolumn{2}{|c|}{ EPoSI2 } \\
\hline & $\mathrm{CP}_{1 \cdot \widehat{M}}$ & $\mathrm{~L}_{1 \cdot \widehat{M}}$ & $\mathrm{CP}_{1 \cdot \widehat{M}}$ & $\mathrm{~L}_{1 \cdot \widehat{M}}$ & $\mathrm{CP}_{1 \cdot \widehat{M}}$ & $\mathrm{~L}_{1 \cdot \widehat{M}}$ & $\mathrm{CP}_{1 \cdot \widehat{M}}$ & $\mathrm{~L}_{1 \cdot \widehat{M}}$ & $\mathrm{CP}_{1 \cdot \widehat{M}}$ & $\mathrm{~L}_{1 \cdot \widehat{M}}$ \\
\hline$\{1,2,3,4,5,6,7\}$ & .952 & 1.25 & 1.00 & 3.33 & 1.00 & 2.51 & .948 & 1.20 & 946 & 1.89 \\
\hline$\{1,2,3,4,5,6,7,8\}$ & .957 & 1.25 & 1.00 & 3.31 & 1.00 & 2.50 & .950 & 1.23 & .952 & 2.00 \\
\hline$\{1,2,3,4,5,7\}$ & .955 & 1.22 & 1.00 & 3.25 & 1.00 & 2.45 & .948 & 1.21 & .948 & 1.34 \\
\hline $\begin{array}{l}\text { Top Selected } \\
\text { Submodels }\end{array}$ & $\mathrm{CP}_{2 \cdot \widehat{M}}$ & $\mathrm{~L}_{2 \cdot \widehat{M}}$ & $\mathrm{CP}_{2 \cdot \widehat{M}}$ & $\mathrm{~L}_{2 \cdot \widehat{M}}$ & $\mathrm{CP}_{2 \cdot \widehat{M}}$ & $\mathrm{~L}_{2 \cdot \widehat{M}}$ & $\mathrm{CP}_{2 \cdot \widehat{M}}$ & $\mathrm{~L}_{2 \cdot \widehat{M}}$ & $\mathrm{CP}_{2 \cdot \widehat{M}}$ & $\mathrm{~L}_{2 \cdot \widehat{M}}$ \\
\hline$\{1,2,3,4,5,6,7\}$ & .949 & 1.29 & 1.00 & 3.44 & 1.00 & 2.59 & .943 & 1.24 & .954 & 1.80 \\
\hline$\{1,2,3,4,5,6,7,8\}$ & .942 & 1.31 & 1.00 & 3.49 & .999 & 2.63 & .929 & 1.26 & .944 & 2.34 \\
\hline$\{1,2,3,4,5,7\}$ & .943 & 1.26 & 1.00 & 3.35 & .999 & 2.53 & .936 & 1.22 & .934 & 1.35 \\
\hline $\begin{array}{l}\text { Top Selected } \\
\text { Submodels }\end{array}$ & $\mathrm{CP}_{3 \cdot \widehat{M}}$ & $\mathrm{~L}_{3 \cdot \widehat{M}}$ & $\mathrm{CP}_{3 \cdot \widehat{M}}$ & $\mathrm{~L}_{3 \cdot \widehat{M}}$ & $\mathrm{CP}_{3 \cdot \widehat{M}}$ & $\mathrm{~L}_{3 \cdot \widehat{M}}$ & $\mathrm{CP}_{3 \cdot \widehat{M}}$ & $\mathrm{~L}_{3 \cdot \widehat{M}}$ & $\mathrm{CP}_{3 \cdot \widehat{M}}$ & $\mathrm{~L}_{3 \cdot \widehat{M}}$ \\
\hline$\{1,2,3,4,5,6,7\}$ & .947 & 1.23 & 1.00 & 3.26 & 1.00 & 2.46 & .939 & 1.19 & .940 & 1.59 \\
\hline$\{1,2,3,4,5,6,7,8\}$ & .948 & 1.24 & 1.00 & 3.28 & 1.00 & 2.48 & .943 & 1.19 & 951 & 1.85 \\
\hline$\{1,2,3,4,5,7\}$ & .952 & 1.21 & 1.00 & 3.23 & .999 & 2.44 & .939 & 1.20 & .939 & 1.32 \\
\hline $\begin{array}{l}\text { Top Selected } \\
\text { Submodels }\end{array}$ & $\mathrm{CP}_{4 \cdot \widehat{M}}$ & $\mathrm{~L}_{4 \cdot \widehat{M}}$ & $\mathrm{CP}_{4 \cdot \widehat{M}}$ & $\mathrm{~L}_{4 \cdot \widehat{M}}$ & $\mathrm{CP}_{4 \cdot \widehat{M}}$ & $\mathrm{~L}_{4 \cdot \widehat{M}}$ & $\mathrm{CP}_{4 \cdot \widehat{M}}$ & $\mathrm{~L}_{4 \cdot \widehat{M}}$ & $\mathrm{CP}_{4 \cdot \widehat{M}}$ & $\mathrm{~L}_{4 \cdot \widehat{M}}$ \\
\hline$\{1,2,3,4,5,6,7\}$ & .952 & 1.13 & 1.00 & 3.01 & .999 & 2.27 & .943 & 1.09 & .951 & 1.71 \\
\hline$\{1,2,3,4,5,6,7,8\}$ & .950 & 1.15 & 1.00 & 3.05 & 1.00 & 2.30 & .935 & 1.11 & .953 & 2.29 \\
\hline$\{1,2,3,4,5,7\}$ & .949 & 1.07 & 1.00 & 2.85 & .999 & 2.15 & .934 & 1.03 & .936 & 1.05 \\
\hline $\begin{array}{c}\text { Top Selected } \\
\text { Submodels }\end{array}$ & $\mathrm{CP}_{5 \cdot \widehat{M}}$ & $\mathrm{~L}_{5 \cdot \widehat{M}}$ & $\mathrm{CP}_{5 \cdot \widehat{M}}$ & $\mathrm{~L}_{5 \cdot \widehat{M}}$ & $\mathrm{CP}_{5 \cdot \widehat{M}}$ & $\mathrm{~L}_{5 \cdot \widehat{M}}$ & $\mathrm{CP}_{5 \cdot \widehat{M}}$ & $\mathrm{~L}_{5 \cdot \widehat{M}}$ & $\mathrm{CP}_{5 \cdot \widehat{M}}$ & $\mathrm{~L}_{5 \cdot \widehat{M}}$ \\
\hline$\{1,2,3,4,5,6,7\}$ & .960 & 1.34 & 1.00 & 3.57 & .999 & 2.69 & .954 & 1.29 & .959 & 2.57 \\
\hline$\{1,2,3,4,5,6,7,8\}$ & .938 & 1.34 & 1.00 & 3.57 & .999 & 2.69 & .933 & 1.45 & .961 & 2.74 \\
\hline$\{1,2,3,4,5,7\}$ & .957 & 1.10 & 1.00 & 2.93 & .999 & 2.21 & .948 & 1.08 & .949 & 1.17 \\
\hline
\end{tabular}

$C P_{j \cdot \widehat{M}}$ and $L_{j \cdot \widehat{M}}, j=1,2,3,4$ and 5, of post-selection confidence intervals based on Model $I V$ and $\boldsymbol{X}_{E p}$. 


\begin{tabular}{|c|c|c|c|c|c|c|c|c|c|c|}
\hline & \multicolumn{10}{|c|}{ Post-selection Confidence Intervals } \\
\hline & \multicolumn{2}{|c|}{ Naïve } & \multicolumn{2}{|c|}{ Scheffé } & \multicolumn{2}{|c|}{ PoSI } & \multicolumn{2}{|c|}{ EPoSI1 } & \multicolumn{2}{|c|}{ EPoSI2 } \\
\hline $\begin{array}{c}\text { Top Selected } \\
\text { Submodels }\end{array}$ & $\mathrm{CP}_{1 \cdot \widehat{M}}$ & $\mathrm{~L}_{1 \cdot \widehat{M}}$ & $\mathrm{CP}_{1 \cdot \widehat{M}}$ & $\mathrm{~L}_{1 \cdot \widehat{M}}$ & $\mathrm{CP}_{1 \cdot \widehat{M}}$ & $\mathrm{~L}_{1 \cdot \widehat{M}}$ & $\mathrm{CP}_{1 \cdot \widehat{M}}$ & $\mathrm{~L}_{1 \cdot \widehat{\boldsymbol{M}}}$ & $\mathrm{CP}_{1 \cdot \widehat{M}}$ & $\mathrm{~L}_{1 \cdot \widehat{M}}$ \\
\hline$\{1,2,3,4,5\}$ & .951 & 5.08 & 1.00 & 13.5 & 1.00 & 10.4 & .944 & 4.90 & .991 & 5.11 \\
\hline$\{1,2,3,4\}$ & .958 & 4.92 & 1.00 & 13.1 & 1.00 & 10.1 & .952 & 4.75 & 1.00 & Inf \\
\hline$\{1,2,3,5\}$ & .959 & 4.93 & 1.00 & 13.2 & 1.00 & 10.1 & .961 & 4.78 & 1.00 & Inf \\
\hline $\begin{array}{l}\text { Top Selected } \\
\text { Submodels }\end{array}$ & $\mathrm{CP}_{2 \cdot \widehat{M}}$ & $\mathrm{~L}_{2 \cdot \widehat{M}}$ & $\mathrm{CP}_{2 \cdot \widehat{M}}$ & $\mathrm{~L}_{2 \cdot \widehat{M}}$ & $\mathrm{CP}_{2 \cdot \widehat{M}}$ & $\mathrm{~L}_{2 \cdot \widehat{M}}$ & $\mathrm{CP}_{2 \cdot \widehat{M}}$ & $\mathrm{~L}_{2 \cdot \widehat{M}}$ & $\mathrm{CP}_{2 \cdot \widehat{M}}$ & $\mathrm{~L}_{2 \cdot \widehat{M}}$ \\
\hline$\{1,2,3,4,5\}$ & .950 & 5.08 & 1.00 & 13.5 & 1.00 & 10.4 & .943 & 4.90 & .962 & 5.14 \\
\hline$\{1,2,3,4\}$ & .955 & 4.92 & 1.00 & 13.1 & 1.00 & 10.1 & .947 & 4.77 & 1.00 & Inf \\
\hline$\{1,2,3,5\}$ & .970 & 4.93 & 1.00 & 13.2 & 1.00 & 10.1 & .960 & 4.76 & 1.00 & Inf \\
\hline $\begin{array}{c}\text { Top Selected } \\
\text { Submodels }\end{array}$ & $\mathrm{CP}_{3 \cdot \widehat{M}}$ & $\mathrm{~L}_{3 \cdot \widehat{M}}$ & $\mathrm{CP}_{3 \cdot \widehat{M}}$ & $\mathrm{~L}_{3 \cdot \widehat{M}}$ & $\mathrm{CP}_{3 \cdot \widehat{M}}$ & $\mathrm{~L}_{3 \cdot \widehat{M}}$ & $\mathrm{CP}_{3 \cdot \widehat{M}}$ & $\mathrm{~L}_{3 \cdot \widehat{\boldsymbol{M}}}$ & $\mathrm{CP}_{3 \cdot \widehat{M}}$ & $\mathrm{~L}_{3 \cdot \widehat{M}}$ \\
\hline$\{1,2,3,4,5\}$ & .952 & 5.08 & 1.00 & 13.5 & 1.00 & 10.4 & .944 & 4.90 & .962 & 5.14 \\
\hline$\{1,2,3,4\}$ & .947 & 4.92 & 1.00 & 13.1 & 1.00 & 10.1 & .936 & 4.77 & 1.00 & Inf \\
\hline$\{1,2,3,5\}$ & .981 & 4.93 & 1.00 & 13.2 & 1.00 & 10.1 & .968 & 4.78 & 1.00 & $\operatorname{Inf}$ \\
\hline $\begin{array}{l}\text { Top Selected } \\
\text { Submodels }\end{array}$ & $\mathrm{CP}_{4 \cdot \widehat{M}}$ & $\mathrm{~L}_{4 \cdot \widehat{M}}$ & $\mathrm{CP}_{4 \cdot \widehat{M}}$ & $\mathrm{~L}_{4 \cdot \widehat{M}}$ & $\mathrm{CP}_{4 \cdot \widehat{M}}$ & $\mathrm{~L}_{4 \cdot \widehat{M}}$ & $\mathrm{CP}_{4 \cdot \widehat{M}}$ & $\mathrm{~L}_{4 \cdot \widehat{M}}$ & $\mathrm{CP}_{4 \cdot \widehat{M}}$ & $\mathrm{~L}_{4 \cdot \widehat{M}}$ \\
\hline$\{1,2,3,4,5\}$ & .977 & 5.08 & 1.00 & 13.5 & 1.00 & 10.4 & .974 & 4.90 & .991 & 5.08 \\
\hline$\{1,2,3,4\}$ & .961 & 4.92 & 1.00 & 13.1 & 1.00 & 10.1 & .958 & 4.77 & 1.00 & Inf \\
\hline$\{1,2,4,5\}$ & .941 & 4.94 & 1.00 & 13.2 & 1.00 & 10.1 & .941 & 4.78 & 1.00 & 5.84 \\
\hline $\begin{array}{l}\text { Top Selected } \\
\text { Submodels }\end{array}$ & $\mathrm{CP}_{5 \cdot \widehat{M}}$ & $\mathrm{~L}_{5 \cdot \widehat{M}}$ & $\mathrm{CP}_{5 \cdot \widehat{M}}$ & $\mathrm{~L}_{5 \cdot \widehat{M}}$ & $\mathrm{CP}_{5 \cdot \widehat{M}}$ & $\mathrm{~L}_{5 \cdot \widehat{M}}$ & $\mathrm{CP}_{5 \cdot \widehat{M}}$ & $\mathrm{~L}_{5 \cdot \widehat{M}}$ & $\mathrm{CP}_{5 \cdot \widehat{M}}$ & $\mathrm{~L}_{5 \cdot \widehat{M}}$ \\
\hline$\{1,2,3,4,5\}$ & .966 & 5.08 & 1.00 & 13.5 & 1.00 & 10.4 & .957 & 4.90 & .962 & 5.06 \\
\hline$\{1,2,3,5\}$ & .969 & 4.93 & 1.00 & 13.2 & 1.00 & 10.1 & .962 & 4.76 & 1.00 & Inf \\
\hline$\{1,2,4,5\}$ & 1.00 & 4.94 & 1.00 & 13.2 & 1.00 & 10.1 & .980 & 4.68 & 1.00 & 5.82 \\
\hline
\end{tabular}

$C P_{j \cdot \widehat{M}}$ and $L_{j \cdot \widehat{M}}, j=1$, 2, 3, 4 and 5, of post-selection confidence intervals based on Model IV and $\boldsymbol{X}_{E c}$. 


\begin{tabular}{|c|c|c|c|c|c|c|c|c|c|c|}
\hline & \multicolumn{10}{|c|}{ Post-selection Confidence Intervals } \\
\hline & \multicolumn{2}{|c|}{ Naïve } & \multicolumn{2}{|c|}{ Scheffé } & \multicolumn{2}{|c|}{ PoSI } & \multicolumn{2}{|c|}{ EPoSI1 } & \multicolumn{2}{|c|}{ EPoSI2 } \\
\hline $\begin{array}{l}\text { Top Selected } \\
\text { Submodels }\end{array}$ & $\mathrm{CP}_{1 \cdot \widehat{M}}$ & $\mathrm{~L}_{1 \cdot \widehat{M}}$ & $\mathrm{CP}_{1 \cdot \widehat{M}}$ & $\mathrm{~L}_{1 \cdot \widehat{M}}$ & $\mathrm{CP}_{1 \cdot \widehat{M}}$ & $\mathrm{~L}_{1 \cdot \widehat{M}}$ & $\mathrm{CP}_{1 \cdot \widehat{M}}$ & $\mathrm{~L}_{1 \cdot \widehat{M}}$ & $\mathrm{CP}_{1 \cdot \widehat{M}}$ & $\mathrm{~L}_{1 \cdot \widehat{M}}$ \\
\hline$\{1,2,3,4,5\}$ & .984 & 6.04 & 1.00 & 13.2 & 1.00 & 10.4 & .984 & 6.69 & .967 & 9.62 \\
\hline$\{1,2,3,4,5,9\}$ & .979 & 6.07 & 1.00 & 13.3 & 1.00 & 10.4 & .974 & 7.33 & .956 & 10.8 \\
\hline$\{1,2,3,4,5,6\}$ & .979 & 6.06 & 1.00 & 13.3 & 1.00 & 10.4 & .979 & 6.17 & .954 & 12.5 \\
\hline $\begin{array}{l}\text { Top Selected } \\
\text { Submodels }\end{array}$ & $\mathrm{CP}_{2 \cdot \widehat{M}}$ & $\mathrm{~L}_{2 \cdot \widehat{M}}$ & $\mathrm{CP}_{2 \cdot \widehat{M}}$ & $\mathrm{~L}_{2 \cdot \widehat{M}}$ & $\mathrm{CP}_{2 \cdot \widehat{M}}$ & $\mathrm{~L}_{2 \cdot \widehat{M}}$ & $\mathrm{CP}_{2 \cdot \widehat{M}}$ & $\mathrm{~L}_{2 \cdot \widehat{M}}$ & $\mathrm{CP}_{2 \cdot \widehat{M}}$ & $\mathrm{~L}_{2 \cdot \widehat{M}}$ \\
\hline$\{1,2,3,4,5\}$ & .973 & 5.75 & 1.00 & 12.6 & 1.00 & 9.89 & .971 & 6.10 & .959 & 10.5 \\
\hline$\{1,2,3,4,5,9\}$ & .972 & 5.78 & 1.00 & 12.7 & .999 & 9.93 & .970 & 7.37 & .960 & 12.1 \\
\hline$\{1,2,3,4,5,6\}$ & .978 & 6.00 & 1.00 & 13.1 & 1.00 & 10.3 & .976 & 5.99 & .980 & 20.7 \\
\hline $\begin{array}{c}\text { Top Selected } \\
\text { Submodels }\end{array}$ & $\mathrm{CP}_{3 \cdot \widehat{M}}$ & $\mathrm{~L}_{3 \cdot \widehat{M}}$ & $\mathrm{CP}_{3 \cdot \widehat{M}}$ & $\mathrm{~L}_{3 \cdot \widehat{M}}$ & $\mathrm{CP}_{3 \cdot \widehat{M}}$ & $\mathrm{~L}_{3 \cdot \widehat{M}}$ & $\mathrm{CP}_{3 \cdot \widehat{M}}$ & $\mathrm{~L}_{3 \cdot \widehat{\boldsymbol{M}}}$ & $\mathrm{CP}_{3 \cdot \widehat{M}}$ & $\mathrm{~L}_{3 \cdot \widehat{M}}$ \\
\hline$\{1,2,3,4,5\}$ & .981 & 6.14 & 1.00 & 13.4 & 1.00 & 10.5 & .980 & 6.24 & .961 & 10.6 \\
\hline$\{1,2,3,4,5,9\}$ & .990 & 6.45 & 1.00 & 14.1 & 1.00 & 11.1 & .990 & 6.43 & .977 & 16.4 \\
\hline$\{1,2,3,4,5,6\}$ & .968 & 6.19 & 1.00 & 13.6 & .998 & 10.6 & .968 & 6.17 & .958 & 14.6 \\
\hline $\begin{array}{l}\text { Top Selected } \\
\text { Submodels }\end{array}$ & $\mathrm{CP}_{4 \cdot \widehat{M}}$ & $\mathrm{~L}_{4 \cdot \widehat{M}}$ & $\mathrm{CP}_{4 \cdot \widehat{M}}$ & $\mathrm{~L}_{4 \cdot \widehat{M}}$ & $\mathrm{CP}_{4 \cdot \widehat{M}}$ & $\mathrm{~L}_{4 \cdot \widehat{M}}$ & $\mathrm{CP}_{4 \cdot \widehat{M}}$ & $\mathrm{~L}_{4 \cdot \widehat{M}}$ & $\mathrm{CP}_{4 \cdot \widehat{M}}$ & $\mathrm{~L}_{4 \cdot \widehat{M}}$ \\
\hline$\{1,2,3,4,5\}$ & .960 & 6.47 & 1.00 & 14.2 & 1.00 & 11.1 & .952 & 8.08 & .950 & 10.5 \\
\hline$\{1,2,3,4,5,9\}$ & .961 & 6.58 & 1.00 & 14.4 & 1.00 & 11.3 & .953 & 9.51 & .954 & 13.9 \\
\hline$\{1,2,3,4,5,6\}$ & .962 & 6.56 & 1.00 & 14.4 & .998 & 11.3 & .962 & 6.55 & .968 & 17.3 \\
\hline $\begin{array}{l}\text { Top Selected } \\
\text { Submodels }\end{array}$ & $\mathrm{CP}_{5 \cdot \widehat{M}}$ & $\mathrm{~L}_{5 \cdot \widehat{M}}$ & $\mathrm{CP}_{5 \cdot \widehat{M}}$ & $\mathrm{~L}_{5 \cdot \widehat{M}}$ & $\mathrm{CP}_{5 \cdot \widehat{M}}$ & $\mathrm{~L}_{5 \cdot \widehat{M}}$ & $\mathrm{CP}_{5 \cdot \widehat{M}}$ & $\mathrm{~L}_{5 \cdot \widehat{M}}$ & $\mathrm{CP}_{5 \cdot \widehat{M}}$ & $\mathrm{~L}_{5 \cdot \widehat{M}}$ \\
\hline$\{1,2,3,4,5\}$ & .977 & 5.92 & 1.00 & 13.0 & 1.00 & 10.2 & .973 & 6.44 & .957 & 10.8 \\
\hline$\{1,2,3,4,5,9\}$ & .989 & 6.57 & 1.00 & 14.4 & 1.00 & 11.3 & .978 & 16.7 & .969 & 21.0 \\
\hline$\{1,2,3,4,5,6\}$ & 1.00 & 13.3 & 1.00 & 29.2 & 1.00 & 22.9 & 1.00 & 15.8 & .998 & 86.0 \\
\hline
\end{tabular}

$C P_{j \cdot \widehat{M}}$ and $L_{j \cdot \widehat{M}}, j=1,2,3,4$ and 5, of post-selection confidence intervals based on Model IV and $\boldsymbol{X}_{R l}$. 
S5.5. $\quad$ Model I $(n=60, p=18)$

\begin{tabular}{|c|c|c|c|c|c|c|c|c|c|c|}
\hline & \multicolumn{10}{|c|}{ Post-selection Confidence Intervals } \\
\hline & \multicolumn{2}{|c|}{ Naïve } & \multicolumn{2}{|c|}{ Scheffé } & \multicolumn{2}{|c|}{ PoSI } & \multicolumn{2}{|c|}{ EPoSI1 } & \multicolumn{2}{|c|}{ EPoSI2 } \\
\hline $\begin{array}{l}\text { Top Selected } \\
\text { Submodels }\end{array}$ & $\mathrm{CP}_{1 \cdot \widehat{M}}$ & $\mathrm{~L}_{1 \cdot \widehat{M}}$ & $\mathrm{CP}_{1 \cdot \widehat{M}}$ & $\mathrm{~L}_{1 \cdot \widehat{M}}$ & $\mathrm{CP}_{1 \cdot \widehat{M}}$ & $\mathrm{~L}_{1 \cdot \widehat{M}}$ & $\mathrm{CP}_{1 \cdot \widehat{M}}$ & $\mathrm{~L}_{1 \cdot \widehat{M}}$ & $\mathrm{CP}_{1 \cdot \widehat{M}}$ & $\mathrm{~L}_{1 \cdot \widehat{M}}$ \\
\hline$\{1,2,3\}$ & .975 & 1.43 & 1.00 & 4.13 & 1.00 & 3.04 & .975 & 1.40 & .975 & 1.40 \\
\hline$\{1,2,3,4\}$ & .937 & 1.47 & 1.00 & 4.23 & 1.00 & 3.11 & .926 & 1.43 & .947 & 1.88 \\
\hline$\{1,2,3,14\}$ & .949 & 1.45 & 1.00 & 4.19 & 1.00 & 3.08 & .949 & 1.42 & .962 & 2.22 \\
\hline $\begin{array}{l}\text { Top Selected } \\
\text { Submodels }\end{array}$ & $\mathrm{CP}_{2 \cdot \widehat{M}}$ & $\mathrm{~L}_{2 \cdot \widehat{M}}$ & $\mathrm{CP}_{2 \cdot \widehat{M}}$ & $\mathrm{~L}_{2 \cdot \widehat{M}}$ & $\mathrm{CP}_{2 \cdot \widehat{M}}$ & $\mathrm{~L}_{2 \cdot \widehat{M}}$ & $\mathrm{CP}_{2 \cdot \widehat{M}}$ & $\mathrm{~L}_{2 \cdot \widehat{M}}$ & $\mathrm{CP}_{2 \cdot \widehat{M}}$ & $\mathrm{~L}_{2 \cdot \widehat{M}}$ \\
\hline$\{1,2,3\}$ & .957 & 1.40 & 1.00 & 4.03 & 1.00 & 2.97 & .951 & 1.37 & .951 & 1.37 \\
\hline$\{1,2,3,4\}$ & .937 & 1.54 & 1.00 & 4.43 & 1.00 & 3.26 & .926 & 1.50 & .937 & 2.86 \\
\hline$\{1,2,3,14\}$ & .962 & 1.51 & 1.00 & 4.36 & 1.00 & 3.21 & .962 & 1.48 & .961 & 3.00 \\
\hline $\begin{array}{l}\text { Top Selected } \\
\text { Submodels }\end{array}$ & $\mathrm{CP}_{3 \cdot \widehat{M}}$ & $\mathrm{~L}_{3 \cdot \widehat{M}}$ & $\mathrm{CP}_{3 \cdot \widehat{M}}$ & $\mathrm{~L}_{3 \cdot \widehat{M}}$ & $\mathrm{CP}_{3 \cdot \widehat{M}}$ & $\mathrm{~L}_{3 \cdot \widehat{M}}$ & $\mathrm{CP}_{3 \cdot \widehat{M}}$ & $\mathrm{~L}_{3 \cdot \widehat{M}}$ & $\mathrm{CP}_{3 \cdot \widehat{M}}$ & $\mathrm{~L}_{3 \cdot \widehat{M}}$ \\
\hline$\{1,2,3\}$ & .963 & 1.48 & 1.00 & 4.28 & 1.00 & 3.15 & .951 & 1.45 & .951 & 1.45 \\
\hline$\{1,2,3,4\}$ & .958 & 1.62 & 1.00 & 4.68 & 1.00 & 3.44 & .937 & 1.59 & .947 & 2.97 \\
\hline$\{1,2,3,14\}$ & .949 & 1.56 & 1.00 & 4.50 & 1.00 & 3.31 & .949 & 1.53 & .961 & 2.76 \\
\hline
\end{tabular}

$C P_{j \cdot \widehat{M}}$ and $L_{j \cdot \widehat{M}}, j=1,2$ and 3, of post-selection confidence intervals based on Model I and $\boldsymbol{X}_{E q}$.

\begin{tabular}{|c|c|c|c|c|c|c|c|c|c|c|}
\hline \multirow[b]{3}{*}{$\begin{array}{l}\text { Top Selected } \\
\text { Submodels }\end{array}$} & \multicolumn{10}{|c|}{ Post-selection Confidence Intervals } \\
\hline & \multicolumn{2}{|c|}{ Naïve } & \multicolumn{2}{|c|}{ Scheffé } & \multicolumn{2}{|c|}{ PoSI } & \multicolumn{2}{|c|}{ EPoSI1 } & \multicolumn{2}{|c|}{ EPoSI2 } \\
\hline & $\mathrm{CP}_{1 \cdot \widehat{M}}$ & $\mathrm{~L}_{1 \cdot \widehat{M}}$ & $\mathrm{CP}_{1 \cdot \widehat{M}}$ & $\mathrm{~L}_{1 \cdot \widehat{M}}$ & $\mathrm{CP}_{1 \cdot \widehat{M}}$ & $\mathrm{~L}_{1 \cdot \widehat{M}}$ & $\mathrm{CP}_{1 \cdot \widehat{M}}$ & $\mathrm{~L}_{1 \cdot \widehat{M}}$ & $\mathrm{CP}_{1 \cdot \widehat{M}}$ & $\mathrm{~L}_{1 \cdot \widehat{M}}$ \\
\hline$\{1,2,3\}$ & .953 & 1.93 & 1.00 & 5.57 & 1.00 & 4.10 & .947 & 1.90 & .947 & 1.90 \\
\hline$\{1,2,3,4\}$ & .949 & 1.94 & 1.00 & 5.59 & 1.00 & 4.11 & .943 & 1.90 & .944 & 2.20 \\
\hline$\{1,2,3,5\}$ & .936 & 1.94 & 1.00 & 5.59 & 1.00 & 4.11 & .928 & 1.90 & .933 & 1.91 \\
\hline $\begin{array}{l}\text { Top Selected } \\
\text { Submodels }\end{array}$ & $\mathrm{CP}_{2 \cdot \widehat{M}}$ & $\mathrm{~L}_{2 \cdot \widehat{M}}$ & $\mathrm{CP}_{2 \cdot \widehat{M}}$ & $\mathrm{~L}_{2 \cdot \widehat{M}}$ & $\mathrm{CP}_{2 \cdot \widehat{M}}$ & $\mathrm{~L}_{2 \cdot \widehat{\boldsymbol{M}}}$ & $\mathrm{CP}_{2 \cdot \widehat{M}}$ & $\mathrm{~L}_{2 \cdot \widehat{M}}$ & $\mathrm{CP}_{2 \cdot \widehat{M}}$ & $\mathrm{~L}_{2 \cdot \widehat{M}}$ \\
\hline$\{1,2,3\}$ & .953 & 2.68 & 1.00 & 7.74 & 1.00 & 5.69 & .948 & 2.65 & .948 & 2.65 \\
\hline$\{1,2,3,4\}$ & .951 & 2.69 & 1.00 & 7.75 & 1.00 & 5.70 & .947 & 2.65 & .947 & 2.66 \\
\hline$\{1,2,3,5\}$ & .941 & 2.69 & 1.00 & 7.77 & 1.00 & 5.71 & .942 & 2.72 & .947 & 2.93 \\
\hline $\begin{array}{l}\text { Top Selected } \\
\text { Submodels }\end{array}$ & $\mathrm{CP}_{3 \cdot \widehat{M}}$ & $\mathrm{~L}_{3 \cdot \widehat{M}}$ & $\mathrm{CP}_{3 \cdot \widehat{M}}$ & $\mathrm{~L}_{3 \cdot \widehat{M}}$ & $\mathrm{CP}_{3 \cdot \widehat{M}}$ & $\mathrm{~L}_{3 \cdot \widehat{M}}$ & $\mathrm{CP}_{3 \cdot \widehat{M}}$ & $\mathrm{~L}_{3 \cdot \widehat{M}}$ & $\mathrm{CP}_{3 \cdot \widehat{M}}$ & $\mathrm{~L}_{3 \cdot \widehat{M}}$ \\
\hline$\{1,2,3\}$ & .944 & 1.78 & 1.00 & 5.13 & 1.00 & 3.77 & .939 & 1.74 & .939 & 1.75 \\
\hline$\{1,2,3,4\}$ & .933 & 2.30 & 1.00 & 6.62 & 1.00 & 4.87 & .968 & 5.32 & .975 & 5.59 \\
\hline$\{1,2,3,5\}$ & .921 & 2.06 & 1.00 & 5.96 & 1.00 & 4.38 & .925 & 2.99 & .968 & 5.16 \\
\hline
\end{tabular}

$C P_{j \cdot \widehat{M}}$ and $L_{j \cdot \widehat{M}}, j=1,2$ and 3, of post-selection confidence intervals based on Model I and $\boldsymbol{X}_{T p}$. 


\begin{tabular}{|c|c|c|c|c|c|c|c|c|c|c|}
\hline & \multicolumn{10}{|c|}{ Post-selection Confidence Intervals } \\
\hline & \multicolumn{2}{|c|}{ Naïve } & \multicolumn{2}{|c|}{ Scheffé } & \multicolumn{2}{|c|}{ PoSI } & \multicolumn{2}{|c|}{ EPoSI1 } & \multicolumn{2}{|c|}{ EPoSI2 } \\
\hline $\begin{array}{l}\text { Top Selected } \\
\text { Submodels }\end{array}$ & $\mathrm{CP}_{1 \cdot \widehat{M}}$ & $\mathrm{~L}_{1 \cdot \widehat{M}}$ & $\mathrm{CP}_{1 \cdot \widehat{M}}$ & $\mathrm{~L}_{1 \cdot \widehat{M}}$ & $\mathrm{CP}_{1 \cdot \widehat{M}}$ & $\mathrm{~L}_{1 \cdot \widehat{M}}$ & $\mathrm{CP}_{1 \cdot \widehat{M}}$ & $\mathrm{~L}_{1 \cdot \widehat{M}}$ & $\mathrm{CP}_{1 \cdot \widehat{M}}$ & $\mathrm{~L}_{1 \cdot \widehat{M}}$ \\
\hline$\{1,2,3,4\}$ & .947 & .825 & 1.00 & 2.38 & 1.00 & 1.68 & .943 & .879 & .944 & 1.02 \\
\hline$\{1,2,3\}$ & .955 & .794 & 1.00 & 2.29 & 1.00 & 1.62 & .951 & .777 & .951 & .777 \\
\hline$\{1,2,3,4,6\}$ & .951 & .826 & 1.00 & 2.38 & .999 & 1.68 & .946 & .807 & .948 & 1.06 \\
\hline $\begin{array}{l}\text { Top Selected } \\
\text { Submodels }\end{array}$ & $\mathrm{CP}_{2 \cdot \widehat{M}}$ & $\mathrm{~L}_{2 \cdot \widehat{M}}$ & $\mathrm{CP}_{2 \cdot \widehat{M}}$ & $\mathrm{~L}_{2 \cdot \widehat{M}}$ & $\mathrm{CP}_{2 \cdot \widehat{M}}$ & $\mathrm{~L}_{2 \cdot \widehat{M}}$ & $\mathrm{CP}_{2 \cdot \widehat{M}}$ & $\mathrm{~L}_{2 \cdot \widehat{M}}$ & $\mathrm{CP}_{2 \cdot \widehat{M}}$ & $\mathrm{~L}_{2 \cdot \widehat{M}}$ \\
\hline$\{1,2,3,4\}$ & .947 & .957 & 1.00 & 2.76 & 1.00 & 1.95 & .942 & .935 & .944 & 1.27 \\
\hline$\{1,2,3\}$ & .940 & .906 & 1.00 & 2.62 & 1.00 & 1.85 & .936 & .887 & .936 & .901 \\
\hline$\{1,2,3,4,6\}$ & .931 & 1.02 & 1.00 & 2.95 & 1.00 & 2.08 & .924 & 1.00 & .969 & 2.36 \\
\hline $\begin{array}{c}\text { Top Selected } \\
\text { Submodels }\end{array}$ & $\mathrm{CP}_{3 \cdot \widehat{M}}$ & $\mathrm{~L}_{3 \cdot \widehat{M}}$ & $\mathrm{CP}_{3 \cdot \widehat{M}}$ & $\mathrm{~L}_{3 \cdot \widehat{M}}$ & $\mathrm{CP}_{3 \cdot \widehat{M}}$ & $\mathrm{~L}_{3 \cdot \widehat{M}}$ & $\mathrm{CP}_{3 \cdot \widehat{M}}$ & $\mathrm{~L}_{3 \cdot \widehat{\boldsymbol{M}}}$ & $\mathrm{CP}_{3 \cdot \widehat{M}}$ & $\mathrm{~L}_{3 \cdot \widehat{M}}$ \\
\hline$\{1,2,3,4\}$ & .951 & .975 & 1.00 & 2.81 & 1.00 & 1.99 & .948 & .954 & .948 & 1.45 \\
\hline$\{1,2,3\}$ & .954 & .850 & 1.00 & 2.45 & 1.00 & 1.73 & .951 & .831 & .951 & .833 \\
\hline$\{1,2,3,4,6\}$ & .928 & 1.04 & 1.00 & 2.98 & 1.00 & 2.11 & .923 & 1.01 & .967 & 2.44 \\
\hline
\end{tabular}

$C P_{j \cdot \widehat{M}}$ and $L_{j \cdot \widehat{M}}, j=1$, 2 and 3, of post-selection confidence intervals based on Model I and $\boldsymbol{X}_{E p}$.

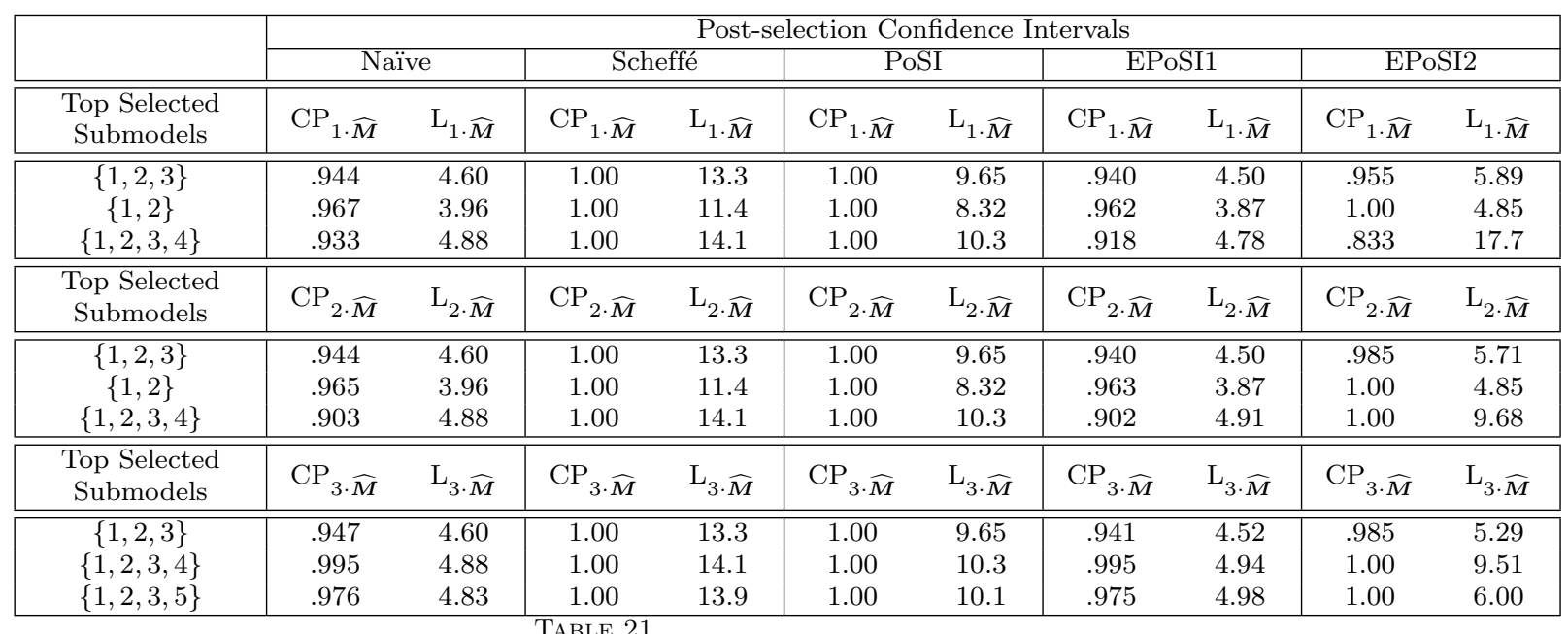

$C P_{j \cdot \widehat{M}}$ and $L_{j \cdot \widehat{M}}, j=1$, 2 and 3, of post-selection confidence intervals based on Model I and $\boldsymbol{X}_{E c}$. 


\begin{tabular}{|c|c|c|c|c|c|c|c|c|c|c|}
\hline \multirow[b]{3}{*}{$\begin{array}{l}\text { Top Selected } \\
\text { Submodels }\end{array}$} & \multicolumn{10}{|c|}{ Post-selection Confidence Intervals } \\
\hline & \multicolumn{2}{|c|}{ Naïve } & \multicolumn{2}{|c|}{ Scheffé } & \multicolumn{2}{|c|}{ PoSI } & \multicolumn{2}{|c|}{ EPoSI1 } & \multicolumn{2}{|c|}{ EPoSI2 } \\
\hline & $\mathrm{CP}_{1 \cdot \widehat{M}}$ & $\mathrm{~L}_{1 \cdot \widehat{M}}$ & $\mathrm{CP}_{1 \cdot \widehat{M}}$ & $\mathrm{~L}_{1 \cdot \widehat{M}}$ & $\mathrm{CP}_{1 \cdot \widehat{M}}$ & $\mathrm{~L}_{1 \cdot \widehat{M}}$ & $\mathrm{CP}_{1 \cdot \widehat{M}}$ & $\mathrm{~L}_{1 \cdot \widehat{M}}$ & $\mathrm{CP}_{1 \cdot \widehat{M}}$ & $\mathrm{~L}_{1 \cdot \widehat{M}}$ \\
\hline$\{1\}$ & .944 & 5.56 & 1.00 & 12.2 & 1.00 & 9.58 & .944 & 5.80 & .978 & 15.4 \\
\hline$\{1,2\}$ & .951 & 5.63 & 1.00 & 12.3 & 1.00 & 9.71 & .953 & 5.93 & .973 & 19.7 \\
\hline$\{1,3\}$ & .956 & 5.65 & 1.00 & 12.4 & 1.00 & 9.73 & .956 & 5.79 & .961 & 19.3 \\
\hline $\begin{array}{l}\text { Top Selected } \\
\text { Submodels }\end{array}$ & $\mathrm{CP}_{2 \cdot \widehat{M}}$ & $\mathrm{~L}_{2 \cdot \widehat{M}}$ & $\mathrm{CP}_{2 \cdot \widehat{M}}$ & $\mathrm{~L}_{2 \cdot \widehat{M}}$ & $\mathrm{CP}_{2 \cdot \widehat{M}}$ & $\mathrm{~L}_{2 \cdot \widehat{M}}$ & $\mathrm{CP}_{2 \cdot \widehat{M}}$ & $\mathrm{~L}_{2 \cdot \widehat{M}}$ & $\mathrm{CP}_{2 \cdot \widehat{M}}$ & $\mathrm{~L}_{2 \cdot \widehat{M}}$ \\
\hline$\{2\}$ & .933 & 5.55 & 1.00 & 12.1 & 1.00 & 9.56 & .932 & 5.63 & .977 & 16.9 \\
\hline$\{1,2\}$ & .965 & 5.63 & 1.00 & 12.3 & 1.00 & 9.71 & .966 & 5.67 & .984 & 19.5 \\
\hline$\{2,3\}$ & .948 & 5.58 & 1.00 & 12.2 & 1.00 & 9.62 & .950 & 5.56 & .970 & 18.5 \\
\hline $\begin{array}{c}\text { Top Selected } \\
\text { Submodels }\end{array}$ & $\mathrm{CP}_{3 \cdot \widehat{M}}$ & $\mathrm{~L}_{3 \cdot \widehat{M}}$ & $\mathrm{CP}_{3 \cdot \widehat{M}}$ & $\mathrm{~L}_{3 \cdot \widehat{M}}$ & $\mathrm{CP}_{3 \cdot \widehat{M}}$ & $\mathrm{~L}_{3 \cdot \widehat{M}}$ & $\mathrm{CP}_{3 \cdot \widehat{M}}$ & $\mathrm{~L}_{3 \cdot \widehat{\boldsymbol{M}}}$ & $\mathrm{CP}_{3 \cdot \widehat{M}}$ & $\mathrm{~L}_{3 \cdot \widehat{M}}$ \\
\hline$\{3\}$ & .940 & 5.55 & 1.00 & 12.2 & 1.00 & 9.57 & .945 & 6.27 & .987 & 16.6 \\
\hline$\{1,3\}$ & .954 & 5.65 & 1.00 & 12.4 & .998 & 9.73 & .954 & 5.95 & .978 & 19.4 \\
\hline$\{2,3\}$ & .943 & 5.58 & 1.00 & 12.2 & 1.00 & 9.62 & .938 & 5.72 & .973 & 19.8 \\
\hline
\end{tabular}

$C P_{j \cdot \widehat{M}}$ and $L_{j \cdot \widehat{M}}, j=1,2$ and 3, of post-selection confidence intervals based on Model I and $\boldsymbol{X}_{R l}$. 
S5.6. Model II $(n=60, p=18)$

\begin{tabular}{|c|c|c|c|c|c|c|c|c|c|c|}
\hline & \multicolumn{10}{|c|}{ Post-selection Confidence Intervals } \\
\hline & \multicolumn{2}{|c|}{ Naïve } & \multicolumn{2}{|c|}{ Scheffé } & \multicolumn{2}{|c|}{ PoSI } & \multicolumn{2}{|c|}{ EPoSI1 } & \multicolumn{2}{|c|}{ EPoSI2 } \\
\hline $\begin{array}{l}\text { Top Selected } \\
\text { Submodels }\end{array}$ & $\mathrm{CP}_{1 \cdot \widehat{M}}$ & $\mathrm{~L}_{1 \cdot \widehat{M}}$ & $\mathrm{CP}_{1 \cdot \widehat{M}}$ & $\mathrm{~L}_{1 \cdot \widehat{M}}$ & $\mathrm{CP}_{1 \cdot \widehat{M}}$ & $\mathrm{~L}_{1 \cdot \widehat{M}}$ & $\mathrm{CP}_{1 \cdot \widehat{M}}$ & $\mathrm{~L}_{1 \cdot \widehat{M}}$ & $\mathrm{CP}_{1 \cdot \widehat{M}}$ & $\mathrm{~L}_{1 \cdot \widehat{M}}$ \\
\hline$\{1,2,3,4,5\}$ & .958 & 1.72 & 1.00 & 4.97 & 1.00 & 3.62 & .958 & 1.68 & .957 & 1.68 \\
\hline$\{1,2,3,4,5,14\}$ & .911 & 1.74 & 1.00 & 5.02 & 1.00 & 3.66 & .899 & 1.70 & .908 & 2.70 \\
\hline$\{1,2,3,4,5,11\}$ & .973 & 1.72 & 1.00 & 4.96 & 1.00 & 3.62 & .973 & 1.68 & .972 & 1.82 \\
\hline $\begin{array}{l}\text { Top Selected } \\
\text { Submodels }\end{array}$ & $\mathrm{CP}_{2 \cdot \widehat{M}}$ & $\mathrm{~L}_{2 \cdot \widehat{M}}$ & $\mathrm{CP}_{2 \cdot \widehat{M}}$ & $\mathrm{~L}_{2 \cdot \widehat{M}}$ & $\mathrm{CP}_{2 \cdot \widehat{M}}$ & $\mathrm{~L}_{2 \cdot \widehat{M}}$ & $\mathrm{CP}_{2 \cdot \widehat{M}}$ & $\mathrm{~L}_{2 \cdot \widehat{M}}$ & $\mathrm{CP}_{2 \cdot \widehat{M}}$ & $\mathrm{~L}_{2 \cdot \widehat{M}}$ \\
\hline$\{1,2,3,4,5\}$ & .958 & 1.82 & 1.00 & 5.24 & 1.00 & 3.82 & .950 & 1.78 & .949 & 1.77 \\
\hline$\{1,2,3,4,5,14\}$ & .949 & 1.92 & 1.00 & 5.52 & 1.00 & 4.03 & .930 & 1.87 & .953 & 3.42 \\
\hline$\{1,2,3,4,5,11\}$ & .946 & 1.83 & 1.00 & 5.27 & 1.00 & 3.84 & .937 & 1.79 & .935 & 2.18 \\
\hline $\begin{array}{l}\text { Top Selected } \\
\text { Submodels }\end{array}$ & $\mathrm{CP}_{3 \cdot \widehat{M}}$ & $\mathrm{~L}_{3 \cdot \widehat{M}}$ & $\mathrm{CP}_{3 \cdot \widehat{M}}$ & $\mathrm{~L}_{3 \cdot \widehat{M}}$ & $\mathrm{CP}_{3 \cdot \widehat{M}}$ & $\mathrm{~L}_{3 \cdot \widehat{M}}$ & $\mathrm{CP}_{3 \cdot \widehat{M}}$ & $\mathrm{~L}_{3 \cdot \widehat{M}}$ & $\mathrm{CP}_{3 \cdot \widehat{M}}$ & $\mathrm{~L}_{3 \cdot \widehat{M}}$ \\
\hline$\{1,2,3,4,5\}$ & .950 & 1.86 & 1.00 & 5.35 & 1.00 & 3.90 & .942 & 1.81 & .940 & 1.81 \\
\hline$\{1,2,3,4,5,14\}$ & .968 & 1.84 & 1.00 & 5.31 & 1.00 & 3.87 & .962 & 1.80 & .961 & 2.56 \\
\hline$\{1,2,3,4,5,11\}$ & .928 & 1.95 & 1.00 & 5.63 & 1.00 & 4.10 & .928 & 1.91 & .944 & 3.38 \\
\hline $\begin{array}{c}\text { Top Selected } \\
\text { Submodels }\end{array}$ & $\mathrm{CP}_{4 \cdot \widehat{M}}$ & $\mathrm{~L}_{4 \cdot \widehat{M}}$ & $\mathrm{CP}_{4 \cdot \widehat{M}}$ & $\mathrm{~L}_{4 \cdot \widehat{M}}$ & $\mathrm{CP}_{4 \cdot \widehat{M}}$ & $\mathrm{~L}_{4 \cdot \widehat{M}}$ & $\mathrm{CP}_{4 \cdot \widehat{M}}$ & $\mathrm{~L}_{4 \cdot \widehat{M}}$ & $\mathrm{CP}_{4 \cdot \widehat{M}}$ & $\mathrm{~L}_{4 \cdot \widehat{M}}$ \\
\hline$\{1,2,3,4,5\}$ & .958 & 1.52 & 1.00 & 4.37 & 1.00 & 3.19 & .958 & 1.48 & .957 & 1.48 \\
\hline$\{1,2,3,4,5,14\}$ & .943 & 1.54 & 1.00 & 4.45 & 1.00 & 3.25 & .937 & 1.51 & .948 & 2.42 \\
\hline$\{1,2,3,4,5,11\}$ & .928 & 1.58 & 1.00 & 4.55 & 1.00 & 3.32 & .919 & 1.54 & .935 & 2.58 \\
\hline $\begin{array}{l}\text { Top Selected } \\
\text { Submodels }\end{array}$ & $\mathrm{CP}_{5 \cdot \widehat{M}}$ & $\mathrm{~L}_{5 \cdot \widehat{M}}$ & $\mathrm{CP}_{5 \cdot \widehat{M}}$ & $\mathrm{~L}_{5 \cdot \widehat{M}}$ & $\mathrm{CP}_{5 \cdot \widehat{M}}$ & $\mathrm{~L}_{5 \cdot \widehat{M}}$ & $\mathrm{CP}_{5 \cdot \widehat{M}}$ & $\mathrm{~L}_{5 \cdot \widehat{M}}$ & $\mathrm{CP}_{5 \cdot \widehat{M}}$ & $\mathrm{~L}_{5 \cdot \widehat{M}}$ \\
\hline$\{1,2,3,4,5\}$ & .983 & 1.57 & 1.00 & 4.52 & 1.00 & 3.30 & .975 & 1.53 & .974 & 1.53 \\
\hline$\{1,2,3,4,5,14\}$ & .943 & 1.70 & 1.00 & 4.89 & 1.00 & 3.57 & .943 & 1.66 & .940 & 3.03 \\
\hline$\{1,2,3,4,5,11\}$ & .946 & 1.60 & 1.00 & 4.62 & 1.00 & 3.37 & .946 & 1.57 & .944 & 2.30 \\
\hline
\end{tabular}

$C P_{j \cdot \widehat{M}}$ and $L_{j \cdot \widehat{M}}, j=1,2,3,4$ and 5, of post-selection confidence intervals based on Model

II and $\boldsymbol{X}_{E q}$. 


\begin{tabular}{|c|c|c|c|c|c|c|c|c|c|c|}
\hline & \multicolumn{10}{|c|}{ Post-selection Confidence Intervals } \\
\hline & \multicolumn{2}{|c|}{ Naïve } & \multicolumn{2}{|c|}{ Scheffé } & \multicolumn{2}{|c|}{ PoSI } & \multicolumn{2}{|c|}{ EPoSI1 } & \multicolumn{2}{|c|}{ EPoSI2 } \\
\hline $\begin{array}{l}\text { Top Selected } \\
\text { Submodels }\end{array}$ & $\mathrm{CP}_{1 \cdot \widehat{M}}$ & $\mathrm{~L}_{1 \cdot \widehat{M}}$ & $\mathrm{CP}_{1 \cdot \widehat{M}}$ & $\mathrm{~L}_{1 \cdot \widehat{M}}$ & $\mathrm{CP}_{1 \cdot \widehat{M}}$ & $\mathrm{~L}_{1 \cdot \widehat{M}}$ & $\mathrm{CP}_{1 \cdot \widehat{M}}$ & $\mathrm{~L}_{1 \cdot \widehat{M}}$ & $\mathrm{CP}_{1 \cdot \widehat{M}}$ & $\mathrm{~L}_{1 \cdot \widehat{M}}$ \\
\hline$\{1,2,3,4,5\}$ & .942 & 1.92 & 1.00 & 5.53 & 1.00 & 4.02 & .936 & 1.89 & .936 & 1.89 \\
\hline$\{1,2,3,4,5,6\}$ & .959 & 1.91 & 1.00 & 5.51 & 1.00 & 4.01 & .953 & 1.87 & .957 & 2.01 \\
\hline$\{1,2,3,4,5,7\}$ & .941 & 1.93 & 1.00 & 5.57 & 1.00 & 4.05 & .937 & 1.96 & .936 & 2.01 \\
\hline $\begin{array}{l}\text { Top Selected } \\
\text { Submodels }\end{array}$ & $\mathrm{CP}_{2 \cdot \widehat{M}}$ & $\mathrm{~L}_{2 \cdot \widehat{M}}$ & $\mathrm{CP}_{2 \cdot \widehat{M}}$ & $\mathrm{~L}_{2 \cdot \widehat{M}}$ & $\mathrm{CP}_{2 \cdot \widehat{M}}$ & $\mathrm{~L}_{2 \cdot \widehat{M}}$ & $\mathrm{CP}_{2 \cdot \widehat{M}}$ & $\mathrm{~L}_{2 \cdot \widehat{M}}$ & $\mathrm{CP}_{2 \cdot \widehat{M}}$ & $\mathrm{~L}_{2 \cdot \widehat{M}}$ \\
\hline$\{1,2,3,4,5\}$ & .948 & 3.11 & 1.00 & 8.96 & 1.00 & 6.52 & .943 & 3.13 & .943 & 3.17 \\
\hline$\{1,2,3,4,5,6\}$ & .952 & 3.10 & 1.00 & 8.93 & 1.00 & 6.49 & .947 & 3.03 & .951 & 3.36 \\
\hline$\{1,2,3,4,5,7\}$ & .949 & 3.13 & 1.00 & 9.03 & 1.00 & 6.56 & .944 & 3.40 & .943 & 3.49 \\
\hline $\begin{array}{l}\text { Top Selected } \\
\text { Submodels }\end{array}$ & $\mathrm{CP}_{3 \cdot \widehat{\boldsymbol{M}}}$ & $\mathrm{L}_{3 \cdot \widehat{M}}$ & $\mathrm{CP}_{3 \cdot \widehat{M}}$ & $\mathrm{~L}_{3 \cdot \widehat{M}}$ & $\mathrm{CP}_{3 \cdot \widehat{M}}$ & $\mathrm{~L}_{3 \cdot \widehat{M}}$ & $\mathrm{CP}_{3 \cdot \widehat{M}}$ & $\mathrm{~L}_{3 \cdot \widehat{M}}$ & $\mathrm{CP}_{3 \cdot \widehat{M}}$ & $\mathrm{~L}_{3 \cdot \widehat{M}}$ \\
\hline$\{1,2,3,4,5\}$ & .944 & 3.09 & 1.00 & 8.91 & 1.00 & 6.48 & .940 & 3.09 & .939 & 3.13 \\
\hline$\{1,2,3,4,5,6\}$ & .938 & 3.10 & 1.00 & 8.94 & 1.00 & 6.50 & .941 & 3.97 & .941 & 4.00 \\
\hline$\{1,2,3,4,5,7\}$ & .953 & 3.13 & 1.00 & 9.02 & 1.00 & 6.56 & .951 & 3.93 & .949 & 4.12 \\
\hline $\begin{array}{c}\text { Top Selected } \\
\text { Submodels }\end{array}$ & $\mathrm{CP}_{4 \cdot \widehat{M}}$ & $\mathrm{~L}_{4 \cdot \widehat{M}}$ & $\mathrm{CP}_{4 \cdot \widehat{M}}$ & $\mathrm{~L}_{4 \cdot \widehat{M}}$ & $\mathrm{CP}_{4 \cdot \widehat{M}}$ & $\mathrm{~L}_{4 \cdot \widehat{M}}$ & $\mathrm{CP}_{4 \cdot \widehat{M}}$ & $\mathrm{~L}_{4 \cdot \widehat{M}}$ & $\mathrm{CP}_{4 \cdot \widehat{M}}$ & $\mathrm{~L}_{4 \cdot \widehat{M}}$ \\
\hline$\{1,2,3,4,5\}$ & .952 & 2.84 & 1.00 & 8.20 & 1.00 & 5.96 & .946 & 2.84 & .946 & 2.87 \\
\hline$\{1,2,3,4,5,6\}$ & .945 & 2.91 & 1.00 & 8.40 & 1.00 & 6.11 & .939 & 2.88 & .951 & 4.52 \\
\hline$\{1,2,3,4,5,7\}$ & .952 & 2.87 & 1.00 & 8.26 & 1.00 & 6.01 & .950 & 2.84 & .953 & 3.14 \\
\hline $\begin{array}{l}\text { Top Selected } \\
\text { Submodels }\end{array}$ & $\mathrm{CP}_{5 \cdot \widehat{M}}$ & $\mathrm{~L}_{5 \cdot \widehat{M}}$ & $\mathrm{CP}_{5 \cdot \widehat{M}}$ & $\mathrm{~L}_{5 \cdot \widehat{M}}$ & $\mathrm{CP}_{5 \cdot \widehat{M}}$ & $\mathrm{~L}_{5 \cdot \widehat{M}}$ & $\mathrm{CP}_{5 \cdot \widehat{M}}$ & $\mathrm{~L}_{5 \cdot \widehat{M}}$ & $\mathrm{CP}_{5 \cdot \widehat{M}}$ & $\mathrm{~L}_{5 \cdot \widehat{M}}$ \\
\hline$\{1,2,3,4,5\}$ & .947 & 2.17 & 1.00 & 6.25 & 1.00 & 4.54 & .942 & 2.13 & .942 & 2.15 \\
\hline$\{1,2,3,4,5,6\}$ & .933 & 3.06 & 1.00 & 8.82 & 1.00 & 6.41 & .926 & 3.21 & .964 & 7.56 \\
\hline$\{1,2,3,4,5,7\}$ & .952 & 2.49 & 1.00 & 7.17 & 1.00 & 5.22 & .965 & 3.68 & .973 & 5.54 \\
\hline
\end{tabular}

$C P_{j \cdot \widehat{M}}$ and $L_{j \cdot \widehat{M}}, j=1,2,3,4$ and 5, of post-selection confidence intervals based on Model II and $\boldsymbol{X}_{T p}$. 


\begin{tabular}{|c|c|c|c|c|c|c|c|c|c|c|}
\hline & \multicolumn{10}{|c|}{ Post-selection Confidence Intervals } \\
\hline & \multicolumn{2}{|c|}{ Naïve } & \multicolumn{2}{|c|}{ Scheffé } & \multicolumn{2}{|c|}{ PoSI } & \multicolumn{2}{|c|}{ EPoSI1 } & \multicolumn{2}{|c|}{ EPoSI2 } \\
\hline $\begin{array}{l}\text { Top Selected } \\
\text { Submodels }\end{array}$ & $\mathrm{CP}_{1 \cdot \widehat{M}}$ & $\mathrm{~L}_{1 \cdot \widehat{M}}$ & $\mathrm{CP}_{1 \cdot \widehat{M}}$ & $\mathrm{~L}_{1 \cdot \widehat{M}}$ & $\mathrm{CP}_{1 \cdot \widehat{M}}$ & $\mathrm{~L}_{1 \cdot \widehat{M}}$ & $\mathrm{CP}_{1 \cdot \widehat{M}}$ & $\mathrm{~L}_{1 \cdot \widehat{M}}$ & $\mathrm{CP}_{1 \cdot \widehat{M}}$ & $\mathrm{~L}_{1 \cdot \widehat{M}}$ \\
\hline$\{1,2,4,5,9\}$ & .966 & .844 & 1.00 & 2.43 & 1.00 & 1.77 & .969 & 1.63 & .965 & 2.00 \\
\hline$\{1,2,4,5\}$ & .945 & .836 & 1.00 & 2.41 & 1.00 & 1.75 & .939 & 1.49 & .942 & 1.69 \\
\hline$\{1,2,3,4,5,9\}$ & .938 & .894 & 1.00 & 2.58 & 1.00 & 1.87 & .928 & 1.09 & .945 & 2.33 \\
\hline $\begin{array}{l}\text { Top Selected } \\
\text { Submodels }\end{array}$ & $\mathrm{CP}_{2 \cdot \widehat{M}}$ & $\mathrm{~L}_{2 \cdot \widehat{M}}$ & $\mathrm{CP}_{2 \cdot \widehat{M}}$ & $\mathrm{~L}_{2 \cdot \widehat{M}}$ & $\mathrm{CP}_{2 \cdot \widehat{M}}$ & $\mathrm{~L}_{2 \cdot \widehat{M}}$ & $\mathrm{CP}_{2 \cdot \widehat{M}}$ & $\mathrm{~L}_{2 \cdot \widehat{M}}$ & $\mathrm{CP}_{2 \cdot \widehat{M}}$ & $\mathrm{~L}_{2 \cdot \widehat{M}}$ \\
\hline$\{1,2,4,5,9\}$ & .972 & .833 & 1.00 & 2.40 & 1.00 & 1.75 & .967 & 2.40 & .966 & 2.61 \\
\hline$\{1,2,4,5\}$ & .964 & .824 & 1.00 & 2.38 & 1.00 & 1.73 & .972 & 2.06 & .968 & 2.15 \\
\hline$\{1,2,3,4,5,9\}$ & .938 & 1.01 & 1.00 & 2.91 & 1.00 & 2.11 & .923 & 1.29 & .975 & 3.45 \\
\hline $\begin{array}{l}\text { Top Selected } \\
\text { Submodels }\end{array}$ & $\mathrm{CP}_{3 \cdot \widehat{M}}$ & $\mathrm{~L}_{3 \cdot \widehat{M}}$ & $\mathrm{CP}_{3 \cdot \widehat{M}}$ & $\mathrm{~L}_{3 \cdot \widehat{M}}$ & $\mathrm{CP}_{3 \cdot \widehat{M}}$ & $\mathrm{~L}_{3 \cdot \widehat{M}}$ & $\mathrm{CP}_{3 \cdot \widehat{M}}$ & $\mathrm{~L}_{3 \cdot \widehat{M}}$ & $\mathrm{CP}_{3 \cdot \widehat{M}}$ & $\mathrm{~L}_{3 \cdot \widehat{M}}$ \\
\hline$\overline{\{1,2,3,4,5,9\}}$ & .891 & 1.00 & 1.00 & 2.89 & 1.00 & 2.10 & .888 & 1.03 & .961 & 3.52 \\
\hline$\{1,2,3,4,5\}$ & .914 & .995 & 1.00 & 2.87 & 1.00 & 2.08 & .901 & .972 & .962 & 3.82 \\
\hline$\{1,2,3,5,9\}$ & .886 & .858 & 1.00 & 2.48 & 1.00 & 1.80 & .883 & .871 & .932 & 3.26 \\
\hline $\begin{array}{l}\text { Top Selected } \\
\text { Submodels }\end{array}$ & $\mathrm{CP}_{4 \cdot \widehat{M}}$ & $\mathrm{~L}_{4 \cdot \widehat{M}}$ & $\mathrm{CP}_{4 \cdot \widehat{M}}$ & $\mathrm{~L}_{4 \cdot \widehat{M}}$ & $\mathrm{CP}_{4 \cdot \widehat{M}}$ & $\mathrm{~L}_{4 \cdot \widehat{M}}$ & $\mathrm{CP}_{4 \cdot \widehat{M}}$ & $\mathrm{~L}_{4 \cdot \widehat{M}}$ & $\mathrm{CP}_{4 \cdot \widehat{M}}$ & $\mathrm{~L}_{4 \cdot \widehat{M}}$ \\
\hline$\{1,2,4,5,9\}$ & .974 & .859 & 1.00 & 2.48 & 1.00 & 1.80 & .965 & 1.02 & .980 & 2.84 \\
\hline$\{1,2,4,5\}$ & .962 & .844 & 1.00 & 2.43 & 1.00 & 1.77 & .970 & 1.98 & .970 & 2.24 \\
\hline$\{1,2,3,4,5,9\}$ & .931 & 1.00 & 1.00 & 2.88 & 1.00 & 2.09 & .923 & 1.08 & .949 & 3.46 \\
\hline $\begin{array}{c}\text { Top Selected } \\
\text { Submodels }\end{array}$ & $\mathrm{CP}_{5 \cdot \widehat{M}}$ & $\mathrm{~L}_{5 \cdot \widehat{M}}$ & $\mathrm{CP}_{5 \cdot \widehat{M}}$ & $\mathrm{~L}_{5 \cdot \widehat{M}}$ & $\mathrm{CP}_{5 \cdot \widehat{M}}$ & $\mathrm{~L}_{5 \cdot \widehat{M}}$ & $\mathrm{CP}_{5 \cdot \widehat{M}}$ & $\mathrm{~L}_{5 \cdot \widehat{M}}$ & $\mathrm{CP}_{5 \cdot \widehat{M}}$ & $\mathrm{~L}_{5 \cdot \widehat{M}}$ \\
\hline$\{1,2,4,5,9\}$ & .959 & .834 & 1.00 & 2.40 & 1.00 & 1.75 & .957 & .895 & .973 & 1.63 \\
\hline$\{1,2,4,5\}$ & .943 & .832 & 1.00 & 2.40 & 1.00 & 1.74 & .933 & .814 & .956 & 1.51 \\
\hline$\{1,2,3,4,5,9\}$ & .952 & .871 & 1.00 & 2.51 & 1.00 & 1.82 & .948 & .871 & .976 & 2.30 \\
\hline
\end{tabular}

$C P_{j \cdot \widehat{M}}$ and $L_{j \cdot \widehat{M}}, j=1,2,3,4$ and 5, of post-selection confidence intervals based on Model

II and $\boldsymbol{X}_{E p}$. 


\begin{tabular}{|c|c|c|c|c|c|c|c|c|c|c|}
\hline & \multicolumn{10}{|c|}{ Post-selection Confidence Intervals } \\
\hline & \multicolumn{2}{|c|}{ Naïve } & \multicolumn{2}{|c|}{ Scheffé } & \multicolumn{2}{|c|}{ PoSI } & \multicolumn{2}{|c|}{ EPoSI1 } & \multicolumn{2}{|c|}{ EPoSI2 } \\
\hline $\begin{array}{l}\text { Top Selected } \\
\text { Submodels }\end{array}$ & $\mathrm{CP}_{1 \cdot \widehat{M}}$ & $\mathrm{~L}_{1 \cdot \widehat{M}}$ & $\mathrm{CP}_{1 \cdot \widehat{M}}$ & $\mathrm{~L}_{1 \cdot \widehat{M}}$ & $\mathrm{CP}_{1 \cdot \widehat{M}}$ & $\mathrm{~L}_{1 \cdot \widehat{M}}$ & $\mathrm{CP}_{1 \cdot \widehat{M}}$ & $\mathrm{~L}_{1 \cdot \widehat{M}}$ & $\mathrm{CP}_{1 \cdot \widehat{M}}$ & $\mathrm{~L}_{1 \cdot \widehat{M}}$ \\
\hline$\{1,2,3,4,5\}$ & .949 & 5.02 & 1.00 & 14.5 & 1.00 & 10.5 & .945 & 4.91 & .946 & 7.48 \\
\hline$\{1,2,3,4\}$ & .956 & 4.89 & 1.00 & 14.1 & 1.00 & 10.2 & .949 & 4.78 & 1.00 & 5.31 \\
\hline$\{1,2,3,5\}$ & .945 & 4.88 & 1.00 & 14.1 & 1.00 & 10.2 & .940 & 4.77 & 1.00 & 5.33 \\
\hline $\begin{array}{l}\text { Top Selected } \\
\text { Submodels }\end{array}$ & $\mathrm{CP}_{2 \cdot \widehat{M}}$ & $\mathrm{~L}_{2 \cdot \widehat{M}}$ & $\mathrm{CP}_{2 \cdot \widehat{M}}$ & $\mathrm{~L}_{2 \cdot \widehat{M}}$ & $\mathrm{CP}_{2 \cdot \widehat{M}}$ & $\mathrm{~L}_{2 \cdot \widehat{M}}$ & $\mathrm{CP}_{2 \cdot \widehat{M}}$ & $\mathrm{~L}_{2 \cdot \widehat{M}}$ & $\mathrm{CP}_{2 \cdot \widehat{M}}$ & $\mathrm{~L}_{2 \cdot \widehat{M}}$ \\
\hline$\{1,2,3,4,5\}$ & .938 & 5.02 & 1.00 & 14.5 & 1.00 & 10.5 & .936 & 5.07 & .946 & 6.49 \\
\hline$\{1,2,3,4\}$ & .945 & 4.89 & 1.00 & 14.1 & 1.00 & 10.2 & .939 & 4.80 & 1.00 & 5.35 \\
\hline$\{1,2,3,5\}$ & .963 & 4.88 & 1.00 & 14.1 & 1.00 & 10.2 & .955 & 4.76 & 1.00 & 5.60 \\
\hline $\begin{array}{l}\text { Top Selected } \\
\text { Submodels }\end{array}$ & $\mathrm{CP}_{3 \cdot \widehat{M}}$ & $\mathrm{~L}_{3 \cdot \widehat{M}}$ & $\mathrm{CP}_{3 \cdot \widehat{M}}$ & $\mathrm{~L}_{3 \cdot \widehat{M}}$ & $\mathrm{CP}_{3 \cdot \widehat{M}}$ & $\mathrm{~L}_{3 \cdot \widehat{M}}$ & $\mathrm{CP}_{3 \cdot \widehat{M}}$ & $\mathrm{~L}_{3 \cdot \widehat{M}}$ & $\mathrm{CP}_{3 \cdot \widehat{M}}$ & $\mathrm{~L}_{3 \cdot \widehat{M}}$ \\
\hline$\{1,2,3,4,5\}$ & .961 & 5.02 & 1.00 & 14.5 & 1.00 & 10.5 & .961 & 5.15 & .973 & 6.44 \\
\hline$\{1,2,3,4\}$ & .971 & 4.89 & 1.00 & 14.1 & 1.00 & 10.2 & .974 & 4.80 & 1.00 & 5.00 \\
\hline$\{1,2,3,5\}$ & .981 & 4.88 & 1.00 & 14.1 & 1.00 & 10.2 & .976 & 4.76 & 1.00 & 5.92 \\
\hline $\begin{array}{l}\text { Top Selected } \\
\text { Submodels }\end{array}$ & $\mathrm{CP}_{4 \cdot \widehat{M}}$ & $\mathrm{~L}_{4 \cdot \widehat{M}}$ & $\mathrm{CP}_{4 \cdot \widehat{M}}$ & $\mathrm{~L}_{4 \cdot \widehat{M}}$ & $\mathrm{CP}_{4 \cdot \widehat{M}}$ & $\mathrm{~L}_{4 \cdot \widehat{M}}$ & $\mathrm{CP}_{4 \cdot \widehat{M}}$ & $\mathrm{~L}_{4 \cdot \widehat{M}}$ & $\mathrm{CP}_{4 \cdot \widehat{M}}$ & $\mathrm{~L}_{4 \cdot \widehat{M}}$ \\
\hline$\{1,2,3,4,5\}$ & .976 & 5.02 & 1.00 & 14.5 & 1.00 & 10.5 & .973 & 5.19 & 1.00 & 5.76 \\
\hline$\{1,2,3,4\}$ & .939 & 4.89 & 1.00 & 14.1 & 1.00 & 10.2 & .934 & 4.78 & 1.00 & 4.92 \\
\hline$\{1,2,4,5\}$ & .989 & 4.90 & 1.00 & 14.1 & 1.00 & 10.3 & .989 & 4.79 & 1.00 & Inf \\
\hline $\begin{array}{l}\text { Top Selected } \\
\text { Submodels }\end{array}$ & $\mathrm{CP}_{5 \cdot \widehat{M}}$ & $\mathrm{~L}_{5 \cdot \widehat{M}}$ & $\mathrm{CP}_{5 \cdot \widehat{M}}$ & $\mathrm{~L}_{5 \cdot \widehat{M}}$ & $\mathrm{CP}_{5 \cdot \widehat{M}}$ & $\mathrm{~L}_{5 \cdot \widehat{M}}$ & $\mathrm{CP}_{5 \cdot \widehat{M}}$ & $\mathrm{~L}_{5 \cdot \widehat{M}}$ & $\mathrm{CP}_{5 \cdot \widehat{M}}$ & $\mathrm{~L}_{5 \cdot \widehat{M}}$ \\
\hline$\{1,2,3,4,5\}$ & .955 & 5.02 & 1.00 & 14.5 & 1.00 & 10.5 & .949 & 4.91 & 1.00 & 5.55 \\
\hline$\{1,2,3,5\}$ & .975 & 4.88 & 1.00 & 14.1 & 1.00 & 10.2 & .971 & 4.77 & 1.00 & 5.22 \\
\hline$\{1,2,4,5\}$ & .995 & 4.90 & 1.00 & 14.1 & 1.00 & 10.3 & .993 & 4.76 & 1.00 & Inf \\
\hline
\end{tabular}

$C P_{j \cdot \widehat{M}}$ and $L_{j \cdot \widehat{M}}, j=1,2,3,4$ and 5, of post-selection confidence intervals based on Model

II and $\boldsymbol{X}_{E c}$. 


\begin{tabular}{|c|c|c|c|c|c|c|c|c|c|c|}
\hline & \multicolumn{10}{|c|}{ Post-selection Confidence Intervals } \\
\hline & \multicolumn{2}{|c|}{ Naïve } & \multicolumn{2}{|c|}{ Scheffé } & \multicolumn{2}{|c|}{ PoSI } & \multicolumn{2}{|c|}{ EPoSI1 } & \multicolumn{2}{|c|}{ EPoSI2 } \\
\hline $\begin{array}{c}\text { Top Selected } \\
\text { Submodels }\end{array}$ & $\mathrm{CP}_{1 \cdot \widehat{M}}$ & $\mathrm{~L}_{1 \cdot \widehat{M}}$ & $\mathrm{CP}_{1 \cdot \widehat{M}}$ & $\mathrm{~L}_{1 \cdot \widehat{M}}$ & $\mathrm{CP}_{1 \cdot \widehat{M}}$ & $\mathrm{~L}_{1 \cdot \widehat{M}}$ & $\mathrm{CP}_{1 \cdot \widehat{M}}$ & $\mathrm{~L}_{1 \cdot \widehat{\boldsymbol{M}}}$ & $\mathrm{CP}_{1 \cdot \widehat{M}}$ & $\mathrm{~L}_{1 \cdot \widehat{M}}$ \\
\hline$\{1,3,4\}$ & .996 & 5.91 & 1.00 & 12.9 & 1.00 & 10.3 & .996 & 6.03 & .996 & 14.9 \\
\hline$\{1,3,4,5\}$ & .984 & 6.02 & 1.00 & 13.2 & 1.00 & 10.5 & .983 & 8.52 & .976 & 18.3 \\
\hline$\{1,2,3,4\}$ & .975 & 5.96 & 1.00 & 13.1 & 1.00 & 10.3 & .974 & 7.09 & .970 & 17.5 \\
\hline $\begin{array}{l}\text { Top Selected } \\
\text { Submodels }\end{array}$ & $\mathrm{CP}_{2 \cdot \widehat{M}}$ & $\mathrm{~L}_{2 \cdot \widehat{M}}$ & $\mathrm{CP}_{2 \cdot \widehat{M}}$ & $\mathrm{~L}_{2 \cdot \widehat{M}}$ & $\mathrm{CP}_{2 \cdot \widehat{M}}$ & $\mathrm{~L}_{2 \cdot \widehat{M}}$ & $\mathrm{CP}_{2 \cdot \widehat{M}}$ & $\mathrm{~L}_{2 \cdot \widehat{M}}$ & $\mathrm{CP}_{2 \cdot \widehat{M}}$ & $\mathrm{~L}_{2 \cdot \widehat{M}}$ \\
\hline$\{1,2,3,4\}$ & .967 & 5.78 & 1.00 & 12.6 & 1.00 & 10.0 & .967 & 6.05 & .990 & 18.3 \\
\hline$\{1,2,3,4,5\}$ & .961 & 5.76 & 1.00 & 12.6 & 1.00 & 10.0 & .947 & 7.95 & .993 & 18.3 \\
\hline$\{1,2,4\}$ & .965 & 5.77 & 1.00 & 12.6 & .993 & 10.0 & .964 & 7.89 & .975 & 17.2 \\
\hline $\begin{array}{c}\text { Top Selected } \\
\text { Submodels }\end{array}$ & $\mathrm{CP}_{3 \cdot \widehat{M}}$ & $\mathrm{~L}_{3 \cdot \widehat{M}}$ & $\mathrm{CP}_{3 \cdot \widehat{M}}$ & $\mathrm{~L}_{3 \cdot \widehat{M}}$ & $\mathrm{CP}_{3 \cdot \widehat{M}}$ & $\mathrm{~L}_{3 \cdot \widehat{M}}$ & $\mathrm{CP}_{3 \cdot \widehat{M}}$ & $\mathrm{~L}_{3 \cdot \widehat{\boldsymbol{M}}}$ & $\mathrm{CP}_{3 \cdot \widehat{M}}$ & $\mathrm{~L}_{3 \cdot \widehat{M}}$ \\
\hline$\{1,3,4\}$ & .966 & 6.06 & 1.00 & 13.3 & 1.00 & 10.5 & .966 & 6.17 & .975 & 16.3 \\
\hline$\{1,3,4,5\}$ & .980 & 6.15 & 1.00 & 13.5 & 1.00 & 10.7 & .980 & 7.03 & .976 & 20.7 \\
\hline$\{1,2,3,4\}$, & .975 & 6.08 & 1.00 & 13.3 & 1.00 & 10.6 & .975 & 6.45 & .975 & 17.5 \\
\hline $\begin{array}{c}\text { Top Selected } \\
\text { Submodels }\end{array}$ & $\mathrm{CP}_{4 \cdot \widehat{M}}$ & $\mathrm{~L}_{4 \cdot \widehat{M}}$ & $\mathrm{CP}_{4 \cdot \widehat{M}}$ & $\mathrm{~L}_{4 \cdot \widehat{M}}$ & $\mathrm{CP}_{4 \cdot \widehat{M}}$ & $\mathrm{~L}_{4 \cdot \widehat{M}}$ & $\mathrm{CP}_{4 \cdot \widehat{M}}$ & $\mathrm{~L}_{4 \cdot \widehat{M}}$ & $\mathrm{CP}_{4 \cdot \widehat{M}}$ & $\mathrm{~L}_{4 \cdot \widehat{M}}$ \\
\hline$\{1,3,4\}$ & .989 & 6.32 & 1.00 & 13.8 & 1.00 & 11.0 & .989 & 6.30 & .980 & 17.8 \\
\hline$\{1,3,4,5\}$ & .984 & 6.36 & 1.00 & 13.9 & 1.00 & 11.0 & .984 & 6.34 & .986 & 20.7 \\
\hline$\{1,2,3,4\}$ & .996 & 6.46 & 1.00 & 14.2 & 1.00 & 11.2 & .996 & 6.80 & .980 & 21.7 \\
\hline $\begin{array}{l}\text { Top Selected } \\
\text { Submodels }\end{array}$ & $\mathrm{CP}_{5 \cdot \widehat{M}}$ & $\mathrm{~L}_{5 \cdot \widehat{M}}$ & $\mathrm{CP}_{5 \cdot \widehat{M}}$ & $\mathrm{~L}_{5 \cdot \widehat{M}}$ & $\mathrm{CP}_{5 \cdot \widehat{M}}$ & $\mathrm{~L}_{5 \cdot \widehat{M}}$ & $\mathrm{CP}_{5 \cdot \widehat{M}}$ & $\mathrm{~L}_{5 \cdot \widehat{M}}$ & $\mathrm{CP}_{5 \cdot \widehat{M}}$ & $\mathrm{~L}_{5 \cdot \widehat{M}}$ \\
\hline$\{1,3,4,5\}$ & .980 & 5.93 & 1.00 & 13.0 & 1.00 & 10.3 & .979 & 9.34 & .976 & 18.9 \\
\hline$\{1,4,5\}$ & .978 & 5.84 & 1.00 & 12.8 & 1.00 & 10.1 & .977 & 8.42 & .987 & 15.1 \\
\hline$\{1,2,3,4,5\}$ & .994 & 5.92 & 1.00 & 13.0 & 1.00 & 10.3 & .994 & 8.27 & .980 & 19.2 \\
\hline
\end{tabular}

$C P_{j \cdot \widehat{M}}$ and $L_{j \cdot \widehat{M}}, j=1,2,3,4$ and 5, of post-selection confidence intervals based on Model II and $\boldsymbol{X}_{R l}$. 
S5.7. $\quad$ Model III $(n=60, p=18)$

\begin{tabular}{|c|c|c|c|c|c|c|c|c|c|c|}
\hline \multirow[b]{3}{*}{$\begin{array}{l}\text { Top Selected } \\
\text { Submodels }\end{array}$} & \multicolumn{10}{|c|}{ Post-selection Confidence Intervals } \\
\hline & \multicolumn{2}{|c|}{ Naïve } & \multicolumn{2}{|c|}{ Scheffé } & \multicolumn{2}{|c|}{ PoSI } & \multicolumn{2}{|c|}{ EPoSI1 } & \multicolumn{2}{|c|}{ EPoSI2 } \\
\hline & $\mathrm{CP}_{1 \cdot \widehat{M}}$ & $\mathrm{~L}_{1 \cdot \widehat{\boldsymbol{M}}}$ & $\mathrm{CP}_{1 \cdot \widehat{M}}$ & $\mathrm{~L}_{1 \cdot \widehat{M}}$ & $\mathrm{CP}_{1 \cdot \widehat{M}}$ & $\mathrm{~L}_{1 \cdot \widehat{M}}$ & $\mathrm{CP}_{1 \cdot \widehat{M}}$ & $\mathrm{~L}_{1 \cdot \widehat{\boldsymbol{M}}}$ & $\mathrm{CP}_{1 \cdot \widehat{M}}$ & $\mathrm{~L}_{1 \cdot \widehat{\boldsymbol{M}}}$ \\
\hline$\{1,2,3\}$ & .947 & 1.27 & 1.00 & 3.65 & 1.00 & 2.66 & .942 & 1.24 & .941 & 1.24 \\
\hline$\{1,2,3,17\}$ & .968 & 1.46 & 1.00 & 4.22 & 1.00 & 3.08 & .968 & 1.43 & .989 & 2.65 \\
\hline$\{1,2,3,13\}$ & .935 & 1.33 & 1.00 & 3.83 & 1.00 & 2.79 & .935 & 1.30 & .944 & 2.11 \\
\hline $\begin{array}{l}\text { Top Selected } \\
\text { Submodels }\end{array}$ & $\mathrm{CP}_{2 \cdot \widehat{M}}$ & $\mathrm{~L}_{2 \cdot \widehat{M}}$ & $\mathrm{CP}_{2 \cdot \widehat{M}}$ & $\mathrm{~L}_{2 \cdot \widehat{M}}$ & $\mathrm{CP}_{2 \cdot \widehat{M}}$ & $\mathrm{~L}_{2 \cdot \widehat{M}}$ & $\mathrm{CP}_{2 \cdot \widehat{M}}$ & $\mathrm{~L}_{2 \cdot \widehat{M}}$ & $\mathrm{CP}_{2 \cdot \widehat{M}}$ & $\mathrm{~L}_{2 \cdot \widehat{M}}$ \\
\hline$\{1,2,3\}$ & .913 & 1.58 & 1.00 & 4.55 & 1.00 & 3.32 & .903 & 1.54 & .902 & 1.54 \\
\hline$\{1,2,3,17\}$ & .989 & 1.61 & 1.00 & 4.65 & 1.00 & 3.39 & .978 & 1.58 & .967 & 2.19 \\
\hline$\{1,2,3,13\}$ & .935 & 1.66 & 1.00 & 4.80 & 1.00 & 3.50 & .925 & 1.63 & .933 & 2.52 \\
\hline $\begin{array}{l}\text { Top Selected } \\
\text { Submodels }\end{array}$ & $\mathrm{CP}_{3 \cdot \widehat{M}}$ & $\mathrm{~L}_{3 \cdot \widehat{M}}$ & $\mathrm{CP}_{3 \cdot \widehat{M}}$ & $\mathrm{~L}_{3 \cdot \widehat{\boldsymbol{M}}}$ & $\mathrm{CP}_{3 \cdot \widehat{M}}$ & $\mathrm{~L}_{3 \cdot \widehat{M}}$ & $\mathrm{CP}_{3 \cdot \widehat{M}}$ & $\mathrm{~L}_{3 \cdot \widehat{M}}$ & $\mathrm{CP}_{3 \cdot \widehat{M}}$ & $\mathrm{~L}_{3 \cdot \widehat{M}}$ \\
\hline$\{1,2,3\}$ & .947 & 1.40 & 1.00 & 4.03 & 1.00 & 2.94 & .942 & 1.36 & .941 & 1.36 \\
\hline$\{1,2,3,17\}$ & .946 & 1.53 & 1.00 & 4.41 & 1.00 & 3.22 & .946 & 1.49 & .944 & 2.67 \\
\hline$\{1,2,3,13\}$ & .968 & 1.49 & 1.00 & 4.30 & 1.00 & 3.14 & .957 & 1.46 & .967 & 2.36 \\
\hline
\end{tabular}

$C P_{j \cdot \widehat{M}}$ and $L_{j \cdot \widehat{M}}, j=1,2$ and 3, of post-selection confidence intervals based on Model III and $\boldsymbol{X}_{E q}$.

\begin{tabular}{|c|c|c|c|c|c|c|c|c|c|c|}
\hline \multirow[b]{3}{*}{$\begin{array}{l}\text { Top Selected } \\
\text { Submodels }\end{array}$} & \multicolumn{10}{|c|}{ Post-selection Confidence Intervals } \\
\hline & \multicolumn{2}{|c|}{ Naïve } & \multicolumn{2}{|c|}{ Scheffé } & \multicolumn{2}{|c|}{ PoSI } & \multicolumn{2}{|c|}{ EPoSI1 } & \multicolumn{2}{|c|}{ EPoSI2 } \\
\hline & $\mathrm{CP}_{1 \cdot \widehat{M}}$ & $\mathrm{~L}_{1 \cdot \widehat{M}}$ & $\mathrm{CP}_{1 \cdot \widehat{M}}$ & $\mathrm{~L}_{1 \cdot \widehat{M}}$ & $\mathrm{CP}_{1 \cdot \widehat{M}}$ & $\mathrm{~L}_{1 \cdot \widehat{\boldsymbol{M}}}$ & $\mathrm{CP}_{1 \cdot \widehat{M}}$ & $\mathrm{~L}_{1 \cdot \widehat{\boldsymbol{M}}}$ & $\mathrm{CP}_{1 \cdot \widehat{M}}$ & $\mathrm{~L}_{1 \cdot \widehat{M}}$ \\
\hline T1,2,3\} & .947 & 1.62 & 1.00 & 4.68 & 1.00 & 3.43 & .942 & 1.59 & .942 & 1.59 \\
\hline$\{1,2,3,4\}$ & .954 & 1.62 & 1.00 & 4.66 & .999 & 3.42 & .950 & 1.61 & .950 & 1.64 \\
\hline$\{1,2,3,5\}$ & .942 & 1.63 & 1.00 & 4.69 & 1.00 & 3.44 & .929 & 1.68 & .931 & 1.85 \\
\hline $\begin{array}{l}\text { Top Selected } \\
\text { Submodels }\end{array}$ & $\mathrm{CP}_{2 \cdot \widehat{M}}$ & $\mathrm{~L}_{2 \cdot \widehat{M}}$ & $\mathrm{CP}_{2 \cdot \widehat{M}}$ & $\mathrm{~L}_{2 \cdot \widehat{M}}$ & $\mathrm{CP}_{2 \cdot \widehat{M}}$ & $\mathrm{~L}_{2 \cdot \widehat{M}}$ & $\mathrm{CP}_{2 \cdot \widehat{M}}$ & $\mathrm{~L}_{2 \cdot \widehat{M}}$ & $\mathrm{CP}_{2 \cdot \widehat{M}}$ & $\mathrm{~L}_{2 \cdot \widehat{M}}$ \\
\hline$\{1,2,3\}$ & .937 & 2.24 & 1.00 & 6.45 & 1.00 & 4.74 & .931 & 2.19 & .931 & 2.19 \\
\hline$\{1,2,3,4\}$ & .948 & 2.23 & 1.00 & 6.44 & 1.00 & 4.73 & .941 & 2.21 & .941 & 2.42 \\
\hline$\{1,2,3,5\}$ & .950 & 2.24 & 1.00 & 6.45 & 1.00 & 4.74 & .940 & 2.19 & .938 & 2.20 \\
\hline $\begin{array}{l}\text { Top Selected } \\
\text { Submodels }\end{array}$ & $\mathrm{CP}_{3 \cdot \widehat{M}}$ & $\mathrm{~L}_{3 \cdot \widehat{M}}$ & $\mathrm{CP}_{3 \cdot \widehat{M}}$ & $\mathrm{~L}_{3 \cdot \widehat{M}}$ & $\mathrm{CP}_{3 \cdot \widehat{M}}$ & $\mathrm{~L}_{3 \cdot \widehat{M}}$ & $\mathrm{CP}_{3 \cdot \widehat{M}}$ & $\mathrm{~L}_{3 \cdot \widehat{M}}$ & $\mathrm{CP}_{3 \cdot \widehat{M}}$ & $\mathrm{~L}_{3 \cdot \widehat{M}}$ \\
\hline$\{1,2,3\}$ & .943 & 1.70 & 1.00 & 4.91 & 1.00 & 3.60 & .939 & 1.66 & .939 & 1.66 \\
\hline$\{1,2,3,4\}$ & .921 & 2.19 & 1.00 & 6.31 & 1.00 & 4.63 & .954 & 3.99 & .967 & 5.23 \\
\hline$\{1,2,3,5\}$ & .918 & 1.96 & 1.00 & 5.65 & 1.00 & 4.15 & .930 & 2.92 & .964 & 4.17 \\
\hline
\end{tabular}

$C P_{j \cdot \widehat{M}}$ and $L_{j \cdot \widehat{M}}, j=1,2$ and 3, of post-selection confidence intervals based on Model III and $\boldsymbol{X}_{T p}$. 


\begin{tabular}{|c|c|c|c|c|c|c|c|c|c|c|}
\hline & \multicolumn{10}{|c|}{ Post-selection Confidence Intervals } \\
\hline & \multicolumn{2}{|c|}{ Naïve } & \multicolumn{2}{|c|}{ Scheffé } & \multicolumn{2}{|c|}{ PoSI } & \multicolumn{2}{|c|}{ EPoSI1 } & \multicolumn{2}{|c|}{ EPoSI2 } \\
\hline $\begin{array}{l}\text { Top Selected } \\
\text { Submodels }\end{array}$ & $\mathrm{CP}_{1 \cdot \widehat{M}}$ & $\mathrm{~L}_{1 \cdot \widehat{M}}$ & $\mathrm{CP}_{1 \cdot \widehat{M}}$ & $\mathrm{~L}_{1 \cdot \widehat{M}}$ & $\mathrm{CP}_{1 \cdot \widehat{M}}$ & $\mathrm{~L}_{1 \cdot \widehat{M}}$ & $\mathrm{CP}_{1 \cdot \widehat{M}}$ & $\mathrm{~L}_{1 \cdot \widehat{M}}$ & $\mathrm{CP}_{1 \cdot \widehat{M}}$ & $\mathrm{~L}_{1 \cdot \widehat{M}}$ \\
\hline$\{1,2,3,4\}$ & .954 & .859 & 1.00 & 2.48 & 1.00 & 1.78 & .950 & .906 & .950 & 1.06 \\
\hline$\{1,2,3\}$ & .953 & .805 & 1.00 & 2.32 & 1.00 & 1.67 & .948 & .788 & .948 & .788 \\
\hline$\{1,2,3,4,11\}$ & .840 & .915 & 1.00 & 2.64 & 1.00 & 1.89 & .839 & .999 & .931 & 2.03 \\
\hline $\begin{array}{l}\text { Top Selected } \\
\text { Submodels }\end{array}$ & $\mathrm{CP}_{2 \cdot \widehat{M}}$ & $\mathrm{~L}_{2 \cdot \widehat{M}}$ & $\mathrm{CP}_{2 \cdot \widehat{M}}$ & $\mathrm{~L}_{2 \cdot \widehat{M}}$ & $\mathrm{CP}_{2 \cdot \widehat{M}}$ & $\mathrm{~L}_{2 \cdot \widehat{M}}$ & $\mathrm{CP}_{2 \cdot \widehat{M}}$ & $\mathrm{~L}_{2 \cdot \widehat{M}}$ & $\mathrm{CP}_{2 \cdot \widehat{M}}$ & $\mathrm{~L}_{2 \cdot \widehat{M}}$ \\
\hline$\{1,2,3,4\}$ & .952 & .924 & 1.00 & 2.67 & 1.00 & 1.91 & .946 & .965 & .947 & 1.13 \\
\hline$\{1,2,3\}$ & .942 & .870 & 1.00 & 2.51 & 1.00 & 1.80 & .933 & .852 & .933 & .852 \\
\hline$\{1,2,3,4,11\}$ & .933 & .935 & 1.00 & 2.70 & 1.00 & 1.93 & .927 & 1.00 & .947 & 1.25 \\
\hline $\begin{array}{l}\text { Top Selected } \\
\text { Submodels }\end{array}$ & $\mathrm{CP}_{3 \cdot \widehat{M}}$ & $\mathrm{~L}_{3 \cdot \widehat{M}}$ & $\mathrm{CP}_{3 \cdot \widehat{M}}$ & $\mathrm{~L}_{3 \cdot \widehat{M}}$ & $\mathrm{CP}_{3 \cdot \widehat{M}}$ & $\mathrm{~L}_{3 \cdot \widehat{M}}$ & $\mathrm{CP}_{3 \cdot \widehat{M}}$ & $\mathrm{~L}_{3 \cdot \widehat{M}}$ & $\mathrm{CP}_{3 \cdot \widehat{M}}$ & $\mathrm{~L}_{3 \cdot \widehat{M}}$ \\
\hline$\{1,2,3,4\}$ & .955 & .966 & 1.00 & 2.79 & 1.00 & 2.00 & .950 & 1.05 & .949 & 1.30 \\
\hline$\{1,2,3\}$ & .937 & .845 & 1.00 & 2.44 & 1.00 & 1.75 & .934 & .827 & .934 & .827 \\
\hline$\{1,2,3,4,11\}$ & .923 & .977 & 1.00 & 2.82 & 1.00 & 2.02 & .898 & 1.08 & .920 & 1.34 \\
\hline
\end{tabular}

$C P_{j \cdot \widehat{M}}$ and $L_{j \cdot \widehat{M}}, j=1,2$ and 3, of post-selection confidence intervals based on Model III and $\boldsymbol{X}_{E p}$.

\begin{tabular}{|c|c|c|c|c|c|c|c|c|c|c|}
\hline & \multicolumn{10}{|c|}{ Post-selection Confidence Intervals } \\
\hline & \multicolumn{2}{|c|}{ Naïve } & \multicolumn{2}{|c|}{ Scheffé } & \multicolumn{2}{|c|}{ PoSI } & \multicolumn{2}{|c|}{ EPoSI1 } & \multicolumn{2}{|c|}{ EPoSI2 } \\
\hline $\begin{array}{l}\text { Top Selected } \\
\text { Submodels }\end{array}$ & $\mathrm{CP}_{1 \cdot \widehat{M}}$ & $\mathrm{~L}_{1 \cdot \widehat{\boldsymbol{M}}}$ & $\mathrm{CP}_{1 \cdot \widehat{M}}$ & $\mathrm{~L}_{1 \cdot \widehat{\boldsymbol{M}}}$ & $\mathrm{CP}_{1 \cdot \widehat{M}}$ & $\mathrm{~L}_{1 \cdot \widehat{M}}$ & $\mathrm{CP}_{1 \cdot \widehat{M}}$ & $\mathrm{~L}_{1 \cdot \widehat{\boldsymbol{M}}}$ & $\mathrm{CP}_{1 \cdot \widehat{M}}$ & $\mathrm{~L}_{1 \cdot \widehat{M}}$ \\
\hline $\begin{array}{c}\{1,2,3\} \\
\{1,2\} \\
\{1,2,3,6\}\end{array}$ & $\begin{array}{l}.948 \\
.961 \\
.922\end{array}$ & $\begin{array}{l}4.60 \\
4.00 \\
4.82\end{array}$ & $\begin{array}{l}1.00 \\
1.00 \\
1.00\end{array}$ & $\begin{array}{l}13.3 \\
11.5 \\
13.9\end{array}$ & $\begin{array}{l}1.00 \\
1.00 \\
1.00\end{array}$ & $\begin{array}{l}9.68 \\
8.41 \\
10.1\end{array}$ & $\begin{array}{l}.943 \\
.949 \\
.904\end{array}$ & $\begin{array}{l}4.50 \\
3.91 \\
4.72\end{array}$ & $\begin{array}{l}.979 \\
1.00 \\
1.00\end{array}$ & $\begin{array}{l}4.57 \\
\text { Inf } \\
\text { Inf }\end{array}$ \\
\hline $\begin{array}{l}\text { Top Selected } \\
\text { Submodels }\end{array}$ & $\mathrm{CP}_{2 \cdot \widehat{M}}$ & $\mathrm{~L}_{2 \cdot \widehat{M}}$ & $\mathrm{CP}_{2 \cdot \widehat{M}}$ & $\mathrm{~L}_{2 \cdot \widehat{M}}$ & $\mathrm{CP}_{2 \cdot \widehat{M}}$ & $\mathrm{~L}_{2 \cdot \widehat{M}}$ & $\mathrm{CP}_{2 \cdot \widehat{M}}$ & $\mathrm{~L}_{2 \cdot \widehat{M}}$ & $\mathrm{CP}_{2 \cdot \widehat{M}}$ & $\mathrm{~L}_{2 \cdot \widehat{M}}$ \\
\hline $\begin{array}{c}\{1,2,3\} \\
\{1,2\} \\
\{1,2,3,6\}\end{array}$ & $\begin{array}{l}.957 \\
.959 \\
.809\end{array}$ & $\begin{array}{l}4.60 \\
4.00 \\
4.82\end{array}$ & $\begin{array}{l}1.00 \\
1.00 \\
1.00\end{array}$ & $\begin{array}{l}13.3 \\
11.5 \\
13.9\end{array}$ & $\begin{array}{l}1.00 \\
1.00 \\
1.00\end{array}$ & $\begin{array}{l}9.68 \\
8.41 \\
10.1\end{array}$ & $\begin{array}{l}.952 \\
.944 \\
.791\end{array}$ & $\begin{array}{l}4.50 \\
3.91 \\
4.72\end{array}$ & $\begin{array}{l}.979 \\
1.00 \\
1.00\end{array}$ & $\begin{array}{l}4.56 \\
\text { Inf } \\
\text { Inf }\end{array}$ \\
\hline $\begin{array}{l}\text { Top Selected } \\
\text { Submodels }\end{array}$ & $\mathrm{CP}_{3 \cdot \widehat{M}}$ & $\mathrm{~L}_{3 \cdot \widehat{M}}$ & $\mathrm{CP}_{3 \cdot \widehat{M}}$ & $\mathrm{~L}_{3 \cdot \widehat{M}}$ & $\mathrm{CP}_{3 \cdot \widehat{M}}$ & $\mathrm{~L}_{3 \cdot \widehat{M}}$ & $\mathrm{CP}_{3 \cdot \widehat{M}}$ & $\mathrm{~L}_{3 \cdot \widehat{M}}$ & $\mathrm{CP}_{3 \cdot \widehat{M}}$ & $\mathrm{~L}_{3 \cdot \widehat{M}}$ \\
\hline $\begin{array}{c}\{1,2,3\} \\
\{1,2,3,6\} \\
\{1,2,3,5\}\end{array}$ & $\begin{array}{l}.969 \\
.913 \\
.918\end{array}$ & $\begin{array}{l}4.60 \\
4.82 \\
4.80\end{array}$ & $\begin{array}{l}1.00 \\
1.00 \\
1.00\end{array}$ & $\begin{array}{l}13.3 \\
13.9 \\
13.8\end{array}$ & $\begin{array}{l}1.00 \\
1.00 \\
1.00\end{array}$ & $\begin{array}{l}9.68 \\
10.1 \\
10.1\end{array}$ & $\begin{array}{l}.967 \\
.894 \\
.891\end{array}$ & $\begin{array}{l}4.50 \\
4.73 \\
4.71 \\
\end{array}$ & $\begin{array}{l}.989 \\
1.00 \\
1.00\end{array}$ & $\begin{array}{c}4.55 \\
\text { Inf } \\
4.88\end{array}$ \\
\hline
\end{tabular}

$C P_{j \cdot \widehat{M}}$ and $L_{j \cdot \widehat{M}}, j=1,2$ and 3, of post-selection confidence intervals based on Model III and $\boldsymbol{X}_{E c}$. 


\begin{tabular}{|c|c|c|c|c|c|c|c|c|c|c|}
\hline \multirow[b]{3}{*}{$\begin{array}{l}\text { Top Selected } \\
\text { Submodels }\end{array}$} & \multicolumn{10}{|c|}{ Post-selection Confidence Intervals } \\
\hline & \multicolumn{2}{|c|}{ Naïve } & \multicolumn{2}{|c|}{ Scheffé } & \multicolumn{2}{|c|}{ PoSI } & \multicolumn{2}{|c|}{ EPoSI1 } & \multicolumn{2}{|c|}{ EPoSI2 } \\
\hline & $\mathrm{CP}_{1 \cdot \widehat{M}}$ & $\mathrm{~L}_{1 \cdot \widehat{M}}$ & $\mathrm{CP}_{1 \cdot \widehat{M}}$ & $\mathrm{~L}_{1 \cdot \widehat{M}}$ & $\mathrm{CP}_{1 \cdot \widehat{M}}$ & $\mathrm{~L}_{1 \cdot \widehat{M}}$ & $\mathrm{CP}_{1 \cdot \widehat{M}}$ & $\mathrm{~L}_{1 \cdot \widehat{M}}$ & $\mathrm{CP}_{1 \cdot \widehat{M}}$ & $\mathrm{~L}_{1 \cdot \widehat{M}}$ \\
\hline$\{1,2,3\}$ & .983 & 5.73 & 1.00 & 12.5 & 1.00 & 9.87 & .982 & 6.06 & .973 & 10.9 \\
\hline$\{1,2,3,4\}$ & .994 & 5.94 & 1.00 & 13.0 & 1.00 & 10.2 & .991 & 7.41 & .977 & 16.5 \\
\hline$\{1,3\}$ & .974 & 5.63 & .998 & 12.3 & .998 & 9.70 & .974 & 5.62 & .948 & 9.96 \\
\hline $\begin{array}{l}\text { Top Selected } \\
\text { Submodels }\end{array}$ & $\mathrm{CP}_{2 \cdot \widehat{M}}$ & $\mathrm{~L}_{2 \cdot \widehat{M}}$ & $\mathrm{CP}_{2 \cdot \widehat{M}}$ & $\mathrm{~L}_{2 \cdot \widehat{M}}$ & $\mathrm{CP}_{2 \cdot \widehat{M}}$ & $\mathrm{~L}_{2 \cdot \widehat{M}}$ & $\mathrm{CP}_{2 \cdot \widehat{M}}$ & $\mathrm{~L}_{2 \cdot \widehat{M}}$ & $\mathrm{CP}_{2 \cdot \widehat{M}}$ & $\mathrm{~L}_{2 \cdot \widehat{M}}$ \\
\hline$\{1,2,3\}$ & .977 & 5.65 & 1.00 & 12.4 & .999 & 9.73 & .977 & 5.69 & .964 & 11.6 \\
\hline$\{1,2,3,4\}$ & .990 & 5.75 & 1.00 & 12.6 & 1.00 & 9.91 & .989 & 6.16 & .984 & 16.0 \\
\hline$\{1,2,3,10\}$ & .987 & 5.72 & 1.00 & 12.5 & 1.00 & 9.86 & .987 & 5.84 & .972 & 14.8 \\
\hline $\begin{array}{l}\text { Top Selected } \\
\text { Submodels }\end{array}$ & $\mathrm{CP}_{3 \cdot \widehat{M}}$ & $\mathrm{~L}_{3 \cdot \widehat{M}}$ & $\mathrm{CP}_{3 \cdot \widehat{M}}$ & $\mathrm{~L}_{3 \cdot \widehat{M}}$ & $\mathrm{CP}_{3 \cdot \widehat{M}}$ & $\mathrm{~L}_{3 \cdot \widehat{M}}$ & $\mathrm{CP}_{3 \cdot \widehat{M}}$ & $\mathrm{~L}_{3 \cdot \widehat{M}}$ & $\mathrm{CP}_{3 \cdot \widehat{M}}$ & $\mathrm{~L}_{3 \cdot \widehat{M}}$ \\
\hline$\{1,2,3\}$ & .979 & 5.66 & 1.00 & 12.4 & 1.00 & 9.75 & .979 & 5.69 & .961 & 11.2 \\
\hline$\{1,2,3,4\}$ & .993 & 6.06 & 1.00 & 13.3 & 1.00 & 10.4 & .993 & 6.73 & .971 & 19.7 \\
\hline$\{1,3\}$ & .985 & 5.63 & 1.00 & 12.3 & 1.00 & 9.70 & .985 & 5.66 & .968 & 10.5 \\
\hline
\end{tabular}

$C P_{j \cdot \widehat{M}}$ and $L_{j \cdot \widehat{M}}, j=1,2$ and 3, of post-selection confidence intervals based on Model III and $\boldsymbol{X}_{R l}$. 
S5.8. $\quad$ Model IV $(n=60, p=18)$

\begin{tabular}{|c|c|c|c|c|c|c|c|c|c|c|}
\hline & \multicolumn{10}{|c|}{ Post-selection Confidence Intervals } \\
\hline & \multicolumn{2}{|c|}{ Naïve } & \multicolumn{2}{|c|}{ Scheffé } & \multicolumn{2}{|c|}{ PoSI } & \multicolumn{2}{|c|}{ EPoSI1 } & \multicolumn{2}{|c|}{ EPoSI2 } \\
\hline $\begin{array}{l}\text { Top Selected } \\
\text { Submodels }\end{array}$ & $\mathrm{CP}_{1 \cdot \widehat{M}}$ & $\mathrm{~L}_{1 \cdot \widehat{M}}$ & $\mathrm{CP}_{1 \cdot \widehat{M}}$ & $\mathrm{~L}_{1 \cdot \widehat{M}}$ & $\mathrm{CP}_{1 \cdot \widehat{M}}$ & $\mathrm{~L}_{1 \cdot \widehat{M}}$ & $\mathrm{CP}_{1 \cdot \widehat{M}}$ & $\mathrm{~L}_{1 \cdot \widehat{M}}$ & $\mathrm{CP}_{1 \cdot \widehat{M}}$ & $\mathrm{~L}_{1 \cdot \widehat{M}}$ \\
\hline$\{1,2,3,4,5\}$ & .972 & 1.52 & 1.00 & 4.38 & 1.00 & 3.18 & .968 & 1.48 & .970 & 1.48 \\
\hline$\{1,2,3,4,5,15\}$ & .932 & 1.56 & 1.00 & 4.49 & 1.00 & 3.26 & .932 & 1.52 & .935 & 1.68 \\
\hline$\{1,2,3,4,5,14\}$ & .964 & 1.56 & 1.00 & 4.50 & 1.00 & 3.27 & .964 & 1.53 & .972 & 1.81 \\
\hline $\begin{array}{l}\text { Top Selected } \\
\text { Submodels }\end{array}$ & $\mathrm{CP}_{2 \cdot \widehat{M}}$ & $\mathrm{~L}_{2 \cdot \widehat{M}}$ & $\mathrm{CP}_{2 \cdot \widehat{M}}$ & $\mathrm{~L}_{2 \cdot \widehat{M}}$ & $\mathrm{CP}_{2 \cdot \widehat{M}}$ & $\mathrm{~L}_{2 \cdot \widehat{M}}$ & $\mathrm{CP}_{2 \cdot \widehat{M}}$ & $\mathrm{~L}_{2 \cdot \widehat{M}}$ & $\mathrm{CP}_{2 \cdot \widehat{M}}$ & $\mathrm{~L}_{2 \cdot \widehat{M}}$ \\
\hline$\{1,2,3,4,5\}$ & .945 & 1.61 & 1.00 & 4.64 & 1.00 & 3.37 & .941 & 1.57 & .935 & 1.57 \\
\hline$\{1,2,3,4,5,15\}$ & .932 & 1.74 & 1.00 & 5.01 & 1.00 & 3.64 & .932 & 1.70 & .927 & 3.11 \\
\hline$\{1,2,3,4,5,14\}$ & .920 & 1.65 & 1.00 & 4.76 & 1.00 & 3.46 & .920 & 1.62 & .916 & 1.89 \\
\hline $\begin{array}{l}\text { Top Selected } \\
\text { Submodels }\end{array}$ & $\mathrm{CP}_{3 \cdot \widehat{M}}$ & $\mathrm{~L}_{3 \cdot \widehat{M}}$ & $\mathrm{CP}_{3 \cdot \widehat{M}}$ & $\mathrm{~L}_{3 \cdot \widehat{M}}$ & $\mathrm{CP}_{3 \cdot \widehat{M}}$ & $\mathrm{~L}_{3 \cdot \widehat{M}}$ & $\mathrm{CP}_{3 \cdot \widehat{M}}$ & $\mathrm{~L}_{3 \cdot \widehat{M}}$ & $\mathrm{CP}_{3 \cdot \widehat{M}}$ & $\mathrm{~L}_{3 \cdot \widehat{M}}$ \\
\hline$\{1,2,3,4,5\}$ & .964 & 1.78 & 1.00 & 5.12 & 1.00 & 3.73 & .957 & 1.74 & .952 & 1.74 \\
\hline$\{1,2,3,4,5,15\}$ & .955 & 1.82 & 1.00 & 5.24 & 1.00 & 3.81 & .955 & 1.78 & .951 & 1.78 \\
\hline$\{1,2,3,4,5,14\}$ & .946 & 1.94 & 1.00 & 5.60 & 1.00 & 4.08 & .946 & 1.90 & .963 & 3.32 \\
\hline $\begin{array}{c}\text { Top Selected } \\
\text { Submodels }\end{array}$ & $\mathrm{CP}_{4 \cdot \widehat{M}}$ & $\mathrm{~L}_{4 \cdot \widehat{M}}$ & $\mathrm{CP}_{4 \cdot \widehat{M}}$ & $\mathrm{~L}_{4 \cdot \widehat{M}}$ & $\mathrm{CP}_{4 \cdot \widehat{M}}$ & $\mathrm{~L}_{4 \cdot \widehat{M}}$ & $\mathrm{CP}_{4 \cdot \widehat{M}}$ & $\mathrm{~L}_{4 \cdot \widehat{M}}$ & $\mathrm{CP}_{4 \cdot \widehat{M}}$ & $\mathrm{~L}_{4 \cdot \widehat{M}}$ \\
\hline$\{1,2,3,4,5\}$ & .945 & 1.64 & 1.00 & 4.72 & .996 & 3.43 & .944 & 1.60 & .939 & 1.60 \\
\hline$\{1,2,3,4,5,15\}$ & .947 & 1.78 & 1.00 & 5.13 & 1.00 & 3.73 & .947 & 1.74 & .959 & 3.27 \\
\hline$\{1,2,3,4,5,14\}$ & .973 & 1.67 & 1.00 & 4.81 & 1.00 & 3.50 & .973 & 1.63 & .972 & 1.63 \\
\hline $\begin{array}{l}\text { Top Selected } \\
\text { Submodels }\end{array}$ & $\mathrm{CP}_{5 \cdot \widehat{M}}$ & $\mathrm{~L}_{5 \cdot \widehat{M}}$ & $\mathrm{CP}_{5 \cdot \widehat{M}}$ & $\mathrm{~L}_{5 \cdot \widehat{M}}$ & $\mathrm{CP}_{5 \cdot \widehat{M}}$ & $\mathrm{~L}_{5 \cdot \widehat{M}}$ & $\mathrm{CP}_{5 \cdot \widehat{M}}$ & $\mathrm{~L}_{5 \cdot \widehat{M}}$ & $\mathrm{CP}_{5 \cdot \widehat{M}}$ & $\mathrm{~L}_{5 \cdot \widehat{M}}$ \\
\hline$\{1,2,3,4,5\}$ & .941 & 1.57 & 1.00 & 4.52 & 1.00 & 3.29 & .933 & 1.53 & .935 & 1.53 \\
\hline$\{1,2,3,4,5,15\}$ & .962 & 1.65 & 1.00 & 4.76 & 1.00 & 3.46 & .952 & 1.61 & .959 & 2.52 \\
\hline$\{1,2,3,4,5,14\}$ & .938 & 1.63 & 1.00 & 4.71 & 1.00 & 3.42 & .936 & 1.59 & .963 & 2.14 \\
\hline
\end{tabular}

$C P_{j \cdot \widehat{M}}$ and $L_{j \cdot \widehat{M}}, j=1,2,3,4$ and 5, of post-selection confidence intervals based on Model

$I V$ and $\boldsymbol{X}_{E q}$. 


\begin{tabular}{|c|c|c|c|c|c|c|c|c|c|c|}
\hline & \multicolumn{10}{|c|}{ Post-selection Confidence Intervals } \\
\hline & \multicolumn{2}{|c|}{ Naïve } & \multicolumn{2}{|c|}{ Scheffé } & \multicolumn{2}{|c|}{ PoSI } & \multicolumn{2}{|c|}{ EPoSI1 } & \multicolumn{2}{|c|}{ EPoSI2 } \\
\hline $\begin{array}{c}\text { Top Selected } \\
\text { Submodels }\end{array}$ & $\mathrm{CP}_{1 \cdot \widehat{M}}$ & $\mathrm{~L}_{1 \cdot \widehat{M}}$ & $\mathrm{CP}_{1 \cdot \widehat{M}}$ & $\mathrm{~L}_{1 \cdot \widehat{M}}$ & $\mathrm{CP}_{1 \cdot \widehat{M}}$ & $\mathrm{~L}_{1 \cdot \widehat{M}}$ & $\mathrm{CP}_{1 \cdot \widehat{M}}$ & $\mathrm{~L}_{1 \cdot \widehat{M}}$ & $\mathrm{CP}_{1 \cdot \widehat{M}}$ & $\mathrm{~L}_{1 \cdot \widehat{M}}$ \\
\hline$\{1,2,3,4,5\}$ & .946 & 1.56 & 1.00 & 4.50 & 1.00 & 3.30 & .939 & 1.52 & .939 & 1.52 \\
\hline$\{1,2,3,4,5,6\}$ & .952 & 1.56 & 1.00 & 4.51 & 1.00 & 3.31 & .945 & 1.53 & .948 & 1.58 \\
\hline$\{1,2,3,4,5,7\}$ & .951 & 1.56 & 1.00 & 4.51 & 1.00 & 3.31 & .948 & 1.53 & .947 & 1.69 \\
\hline $\begin{array}{l}\text { Top Selected } \\
\text { Submodels }\end{array}$ & $\mathrm{CP}_{2 \cdot \widehat{M}}$ & $\mathrm{~L}_{2 \cdot \widehat{M}}$ & $\mathrm{CP}_{2 \cdot \widehat{M}}$ & $\mathrm{~L}_{2 \cdot \widehat{M}}$ & $\mathrm{CP}_{2 \cdot \widehat{M}}$ & $\mathrm{~L}_{2 \cdot \widehat{M}}$ & $\mathrm{CP}_{2 \cdot \widehat{M}}$ & $\mathrm{~L}_{2 \cdot \widehat{M}}$ & $\mathrm{CP}_{2 \cdot \widehat{M}}$ & $\mathrm{~L}_{2 \cdot \widehat{M}}$ \\
\hline$\{1,2,3,4,5\}$ & .940 & 2.00 & 1.00 & 5.77 & 1.00 & 4.23 & .936 & 1.96 & .936 & 1.96 \\
\hline$\{1,2,3,4,5,6\}$ & .946 & 2.02 & 1.00 & 5.81 & .999 & 4.26 & .940 & 2.13 & .937 & 2.54 \\
\hline$\{1,2,3,4,5,7\}$ & .945 & 2.01 & 1.00 & 5.78 & 1.00 & 4.24 & .940 & 1.96 & .930 & 2.13 \\
\hline $\begin{array}{c}\text { Top Selected } \\
\text { Submodels }\end{array}$ & $\mathrm{CP}_{3 \cdot \widehat{M}}$ & $\mathrm{~L}_{3 \cdot \widehat{M}}$ & $\mathrm{CP}_{3 \cdot \widehat{M}}$ & $\mathrm{~L}_{3 \cdot \widehat{M}}$ & $\mathrm{CP}_{3 \cdot \widehat{M}}$ & $\mathrm{~L}_{3 \cdot \widehat{M}}$ & $\mathrm{CP}_{3 \cdot \widehat{M}}$ & $\mathrm{~L}_{3 \cdot \widehat{\boldsymbol{M}}}$ & $\mathrm{CP}_{3 \cdot \widehat{M}}$ & $\mathrm{~L}_{3 \cdot \widehat{M}}$ \\
\hline$\{1,2,3,4,5\}$ & .951 & 2.07 & 1.00 & 5.96 & 1.00 & 4.38 & .946 & 2.02 & .946 & 2.02 \\
\hline$\{1,2,3,4,5,6\}$ & .946 & 2.08 & 1.00 & 6.01 & 1.00 & 4.41 & .939 & 2.04 & .947 & 2.63 \\
\hline$\{1,2,3,4,5,7\}$ & .951 & 2.07 & 1.00 & 5.98 & 1.00 & 4.38 & .951 & 2.03 & .947 & 2.12 \\
\hline $\begin{array}{c}\text { Top Selected } \\
\text { Submodels }\end{array}$ & $\mathrm{CP}_{4 \cdot \widehat{M}}$ & $\mathrm{~L}_{4 \cdot \widehat{M}}$ & $\mathrm{CP}_{4 \cdot \widehat{M}}$ & $\mathrm{~L}_{4 \cdot \widehat{M}}$ & $\mathrm{CP}_{4 \cdot \widehat{M}}$ & $\mathrm{~L}_{4 \cdot \widehat{M}}$ & $\mathrm{CP}_{4 \cdot \widehat{M}}$ & $\mathrm{~L}_{4 \cdot \widehat{M}}$ & $\mathrm{CP}_{4 \cdot \widehat{M}}$ & $\mathrm{~L}_{4 \cdot \widehat{M}}$ \\
\hline$\{1,2,3,4,5\}$ & .942 & 2.03 & 1.00 & 5.86 & 1.00 & 4.30 & .938 & 1.99 & .938 & 1.99 \\
\hline$\{1,2,3,4,5,6\}$ & .960 & 2.04 & 1.00 & 5.88 & 1.00 & 4.31 & .951 & 2.08 & .950 & 2.40 \\
\hline$\{1,2,3,4,5,7\}$ & .973 & 2.04 & 1.00 & 5.87 & 1.00 & 4.31 & .967 & 1.99 & .965 & 2.26 \\
\hline $\begin{array}{c}\text { Top Selected } \\
\text { Submodels }\end{array}$ & $\mathrm{CP}_{5 \cdot \widehat{M}}$ & $\mathrm{~L}_{5 \cdot \widehat{M}}$ & $\mathrm{CP}_{5 \cdot \widehat{M}}$ & $\mathrm{~L}_{5 \cdot \widehat{M}}$ & $\mathrm{CP}_{5 \cdot \widehat{M}}$ & $\mathrm{~L}_{5 \cdot \widehat{M}}$ & $\mathrm{CP}_{5 \cdot \widehat{M}}$ & $\mathrm{~L}_{5 \cdot \widehat{M}}$ & $\mathrm{CP}_{5 \cdot \widehat{M}}$ & $\mathrm{~L}_{5 \cdot \widehat{M}}$ \\
\hline$\{1,2,3,4,5\}$ & .948 & 1.48 & 1.00 & 4.26 & 1.00 & 3.13 & .945 & 1.44 & .945 & 1.44 \\
\hline$\{1,2,3,4,5,6\}$ & .914 & 1.86 & 1.00 & 5.36 & .999 & 3.93 & .919 & 1.91 & .956 & 4.55 \\
\hline$\{1,2,3,4,5,7\}$ & .931 & 1.70 & 1.00 & 4.90 & 1.00 & 3.59 & .934 & 1.80 & .960 & 3.79 \\
\hline
\end{tabular}

$C P_{j \cdot \widehat{M}}$ and $L_{j \cdot \widehat{M}}, j=1,2,3,4$ and 5, of post-selection confidence intervals based on Model IV and $\boldsymbol{X}_{T p}$. 


\begin{tabular}{|c|c|c|c|c|c|c|c|c|c|c|}
\hline & \multicolumn{10}{|c|}{ Post-selection Confidence Intervals } \\
\hline & \multicolumn{2}{|c|}{ Naïve } & \multicolumn{2}{|c|}{ Scheffé } & \multicolumn{2}{|c|}{ PoSI } & \multicolumn{2}{|c|}{ EPoSI1 } & \multicolumn{2}{|c|}{ EPoSI2 } \\
\hline $\begin{array}{l}\text { Top Selected } \\
\text { Submodels }\end{array}$ & $\mathrm{CP}_{1 \cdot \widehat{M}}$ & $\mathrm{~L}_{1 \cdot \widehat{M}}$ & $\mathrm{CP}_{1 \cdot \widehat{M}}$ & $\mathrm{~L}_{1 \cdot \widehat{M}}$ & $\mathrm{CP}_{1 \cdot \widehat{M}}$ & $\mathrm{~L}_{1 \cdot \widehat{M}}$ & $\mathrm{CP}_{1 \cdot \widehat{M}}$ & $\mathrm{~L}_{1 \cdot \widehat{M}}$ & $\mathrm{CP}_{1 \cdot \widehat{M}}$ & $\mathrm{~L}_{1 \cdot \widehat{M}}$ \\
\hline$\{1,2,3,4,5\}$ & .941 & .890 & 1.00 & 2.57 & .999 & 1.81 & .936 & .870 & .936 & .870 \\
\hline$\{1,2,3,4,5,6\}$ & .948 & .893 & 1.00 & 2.58 & 1.00 & 1.81 & .942 & .873 & .943 & .965 \\
\hline$\{1,2,3,4,5,7\}$ & .931 & .893 & 1.00 & 2.57 & 1.00 & 1.81 & .928 & .873 & .930 & 1.09 \\
\hline $\begin{array}{l}\text { Top Selected } \\
\text { Submodels }\end{array}$ & $\mathrm{CP}_{2 \cdot \widehat{M}}$ & $\mathrm{~L}_{2 \cdot \widehat{M}}$ & $\mathrm{CP}_{2 \cdot \widehat{M}}$ & $\mathrm{~L}_{2 \cdot \widehat{M}}$ & $\mathrm{CP}_{2 \cdot \widehat{M}}$ & $\mathrm{~L}_{2 \cdot \widehat{M}}$ & $\mathrm{CP}_{2 \cdot \widehat{M}}$ & $\mathrm{~L}_{2 \cdot \widehat{M}}$ & $\mathrm{CP}_{2 \cdot \widehat{M}}$ & $\mathrm{~L}_{2 \cdot \widehat{M}}$ \\
\hline$\{1,2,3,4,5\}$ & .939 & 1.01 & 1.00 & 2.91 & 1.00 & 2.05 & .934 & .987 & .934 & .987 \\
\hline$\{1,2,3,4,5,6\}$ & .948 & 1.02 & 1.00 & 2.95 & .999 & 2.08 & .944 & .999 & .947 & 1.41 \\
\hline$\{1,2,3,4,5,14\}$ & .934 & 1.02 & 1.00 & 2.95 & 1.00 & 2.08 & .929 & 1.00 & .945 & 1.55 \\
\hline $\begin{array}{l}\text { Top Selected } \\
\text { Submodels }\end{array}$ & $\mathrm{CP}_{3 \cdot \widehat{M}}$ & $\mathrm{~L}_{3 \cdot \widehat{M}}$ & $\mathrm{CP}_{3 \cdot \widehat{M}}$ & $\mathrm{~L}_{3 \cdot \widehat{M}}$ & $\mathrm{CP}_{3 \cdot \widehat{M}}$ & $\mathrm{~L}_{3 \cdot \widehat{M}}$ & $\mathrm{CP}_{3 \cdot \widehat{M}}$ & $\mathrm{~L}_{3 \cdot \widehat{M}}$ & $\mathrm{CP}_{3 \cdot \widehat{M}}$ & $\mathrm{~L}_{3 \cdot \widehat{M}}$ \\
\hline$\overline{\{1,2,3,4,5\}}$ & .949 & .993 & 1.00 & 2.86 & 1.00 & 2.02 & .943 & .970 & .943 & .971 \\
\hline$\{1,2,3,4,5,6\}$ & .951 & .998 & 1.00 & 2.88 & .999 & 2.03 & .944 & .981 & .947 & 1.17 \\
\hline$\{1,2,3,4,5,14\}$ & .951 & 1.01 & 1.00 & 2.92 & .998 & 2.06 & .946 & .999 & .949 & 1.67 \\
\hline $\begin{array}{l}\text { Top Selected } \\
\text { Submodels }\end{array}$ & $\mathrm{CP}_{4 \cdot \widehat{M}}$ & $\mathrm{~L}_{4 \cdot \widehat{M}}$ & $\mathrm{CP}_{4 \cdot \widehat{M}}$ & $\mathrm{~L}_{4 \cdot \widehat{M}}$ & $\mathrm{CP}_{4 \cdot \widehat{M}}$ & $\mathrm{~L}_{4 \cdot \widehat{M}}$ & $\mathrm{CP}_{4 \cdot \widehat{M}}$ & $\mathrm{~L}_{4 \cdot \widehat{M}}$ & $\mathrm{CP}_{4 \cdot \widehat{M}}$ & $\mathrm{~L}_{4 \cdot \widehat{M}}$ \\
\hline$\{1,2,3,4,5\}$ & .951 & 1.14 & 1.00 & 3.27 & .999 & 2.31 & .945 & 1.11 & .945 & 1.12 \\
\hline$\{1,2,3,4,5,6\}$ & .941 & 1.18 & 1.00 & 3.41 & 1.00 & 2.40 & .936 & 1.16 & .948 & 2.04 \\
\hline$\{1,2,3,4,5,14\}$ & .945 & 1.16 & 1.00 & 3.34 & 1.00 & 2.35 & .941 & 1.14 & .946 & 1.91 \\
\hline $\begin{array}{c}\text { Top Selected } \\
\text { Submodels }\end{array}$ & $\mathrm{CP}_{5 \cdot \widehat{M}}$ & $\mathrm{~L}_{5 \cdot \widehat{M}}$ & $\mathrm{CP}_{5 \cdot \widehat{M}}$ & $\mathrm{~L}_{5 \cdot \widehat{M}}$ & $\mathrm{CP}_{5 \cdot \widehat{M}}$ & $\mathrm{~L}_{5 \cdot \widehat{M}}$ & $\mathrm{CP}_{5 \cdot \widehat{M}}$ & $\mathrm{~L}_{5 \cdot \widehat{M}}$ & $\mathrm{CP}_{5 \cdot \widehat{M}}$ & $\mathrm{~L}_{5 \cdot \widehat{M}}$ \\
\hline$\{1,2,3,4,5\}$ & .953 & 1.01 & 1.00 & 2.92 & 1.00 & 2.06 & .950 & .990 & .950 & .991 \\
\hline$\{1,2,3,4,5,6\}$ & .928 & 1.15 & 1.00 & 3.32 & 1.00 & 2.34 & .922 & 1.13 & .958 & 2.38 \\
\hline$\{1,2,3,4,5,14\}$ & .950 & 1.02 & 1.00 & 2.93 & 1.00 & 2.06 & .944 & .992 & .946 & 1.02 \\
\hline
\end{tabular}

$C P_{j \cdot \widehat{M}}$ and $L_{j \cdot \widehat{M}}, j=1,2,3,4$ and 5, of post-selection confidence intervals based on Model $I V$ and $\boldsymbol{X}_{E p}$. 


\begin{tabular}{|c|c|c|c|c|c|c|c|c|c|c|}
\hline & \multicolumn{10}{|c|}{ Post-selection Confidence Intervals } \\
\hline & \multicolumn{2}{|c|}{ Naïve } & \multicolumn{2}{|c|}{ Scheffé } & \multicolumn{2}{|c|}{ PoSI } & \multicolumn{2}{|c|}{ EPoSI1 } & \multicolumn{2}{|c|}{ EPoSI2 } \\
\hline $\begin{array}{l}\text { Top Selected } \\
\text { Submodels }\end{array}$ & $\mathrm{CP}_{1 \cdot \widehat{M}}$ & $\mathrm{~L}_{1 \cdot \widehat{M}}$ & $\mathrm{CP}_{1 \cdot \widehat{M}}$ & $\mathrm{~L}_{1 \cdot \widehat{M}}$ & $\mathrm{CP}_{1 \cdot \widehat{M}}$ & $\mathrm{~L}_{1 \cdot \widehat{M}}$ & $\mathrm{CP}_{1 \cdot \widehat{M}}$ & $\mathrm{~L}_{1 \cdot \widehat{M}}$ & $\mathrm{CP}_{1 \cdot \widehat{M}}$ & $\mathrm{~L}_{1 \cdot \widehat{M}}$ \\
\hline$\{1,2,3,4,5\}$ & .947 & 5.04 & 1.00 & 14.5 & 1.00 & 10.6 & .942 & 4.95 & 1.00 & 4.93 \\
\hline$\{1,2,3,4\}$ & .951 & 4.87 & 1.00 & 14.1 & 1.00 & 10.2 & .971 & 4.95 & 1.00 & Inf \\
\hline$\{1,2,3,5\}$ & .947 & 4.84 & 1.00 & 14.0 & 1.00 & 10.1 & .872 & 4.71 & 1.00 & $\operatorname{Inf}$ \\
\hline $\begin{array}{l}\text { Top Selected } \\
\text { Submodels }\end{array}$ & $\mathrm{CP}_{2 \cdot \widehat{M}}$ & $\mathrm{~L}_{2 \cdot \widehat{M}}$ & $\mathrm{CP}_{2 \cdot \widehat{M}}$ & $\mathrm{~L}_{2 \cdot \widehat{M}}$ & $\mathrm{CP}_{2 \cdot \widehat{M}}$ & $\mathrm{~L}_{2 \cdot \widehat{M}}$ & $\mathrm{CP}_{2 \cdot \widehat{M}}$ & $\mathrm{~L}_{2 \cdot \widehat{M}}$ & $\mathrm{CP}_{2 \cdot \widehat{M}}$ & $\mathrm{~L}_{2 \cdot \widehat{M}}$ \\
\hline$\{1,2,3,4,5\}$ & .948 & 5.04 & 1.00 & 14.5 & 1.00 & 10.6 & .939 & 4.95 & 1.00 & 5.03 \\
\hline$\{1,2,3,4\}$ & .955 & 4.87 & 1.00 & 14.1 & 1.00 & 10.2 & 1.00 & $\operatorname{Inf}$ & 1.00 & Inf \\
\hline$\{1,2,3,5\}$ & .975 & 4.84 & 1.00 & 14.0 & 1.00 & 10.1 & 1.00 & 4.70 & 1.00 & Inf \\
\hline $\begin{array}{l}\text { Top Selected } \\
\text { Submodels }\end{array}$ & $\mathrm{CP}_{3 \cdot \widehat{M}}$ & $\mathrm{~L}_{3 \cdot \widehat{M}}$ & $\mathrm{CP}_{3 \cdot \widehat{M}}$ & $\mathrm{~L}_{3 \cdot \widehat{M}}$ & $\mathrm{CP}_{3 \cdot \widehat{M}}$ & $\mathrm{~L}_{3 \cdot \widehat{M}}$ & $\mathrm{CP}_{3 \cdot \widehat{M}}$ & $\mathrm{~L}_{3 \cdot \widehat{M}}$ & $\mathrm{CP}_{3 \cdot \widehat{M}}$ & $\mathrm{~L}_{3 \cdot \widehat{M}}$ \\
\hline$\{1,2,3,4,5\}$ & .951 & 5.04 & 1.00 & 14.5 & 1.00 & 10.6 & .948 & 4.95 & 1.00 & 4.93 \\
\hline$\{1,2,3,4\}$ & .960 & 4.87 & 1.00 & 14.1 & 1.00 & 10.2 & .941 & 4.94 & 1.00 & Inf \\
\hline$\{1,2,3,5\}$ & .976 & 4.84 & 1.00 & 14.0 & 1.00 & 10.1 & .923 & 4.70 & 1.00 & Inf \\
\hline $\begin{array}{l}\text { Top Selected } \\
\text { Submodels }\end{array}$ & $\mathrm{CP}_{4 \cdot \widehat{M}}$ & $\mathrm{~L}_{4 \cdot \widehat{M}}$ & $\mathrm{CP}_{4 \cdot \widehat{M}}$ & $\mathrm{~L}_{4 \cdot \widehat{M}}$ & $\mathrm{CP}_{4 \cdot \widehat{M}}$ & $\mathrm{~L}_{4 \cdot \widehat{M}}$ & $\mathrm{CP}_{4 \cdot \widehat{M}}$ & $\mathrm{~L}_{4 \cdot \widehat{M}}$ & $\mathrm{CP}_{4 \cdot \widehat{M}}$ & $\mathrm{~L}_{4 \cdot \widehat{M}}$ \\
\hline$\{1,2,3,4,5\}$ & .978 & 5.04 & 1.00 & 14.5 & 1.00 & 10.6 & 1.00 & 4.91 & 1.00 & 4.90 \\
\hline$\{1,2,3,4\}$ & .958 & 4.87 & 1.00 & 14.1 & 1.00 & 10.2 & 1.00 & $\operatorname{Inf}$ & 1.00 & Inf \\
\hline$\{1,2,4,5\}$ & .930 & 4.83 & 1.00 & 13.9 & 1.00 & 10.1 & 1.00 & $\operatorname{Inf}$ & 1.00 & Inf \\
\hline $\begin{array}{l}\text { Top Selected } \\
\text { Submodels }\end{array}$ & $\mathrm{CP}_{5 \cdot \widehat{M}}$ & $\mathrm{~L}_{5 \cdot \widehat{M}}$ & $\mathrm{CP}_{5 \cdot \widehat{M}}$ & $\mathrm{~L}_{5 \cdot \widehat{M}}$ & $\mathrm{CP}_{5 \cdot \widehat{M}}$ & $\mathrm{~L}_{5 \cdot \widehat{M}}$ & $\mathrm{CP}_{5 \cdot \widehat{M}}$ & $\mathrm{~L}_{5 \cdot \widehat{M}}$ & $\mathrm{CP}_{5 \cdot \widehat{M}}$ & $\mathrm{~L}_{5 \cdot \widehat{M}}$ \\
\hline$\{1,2,3,4,5\}$ & .960 & 5.04 & 1.00 & 14.5 & 1.00 & 10.6 & .976 & 4.91 & .875 & 4.89 \\
\hline$\{1,2,3,5\}$ & .976 & 4.84 & 1.00 & 14.0 & 1.00 & 10.1 & 1.00 & 4.70 & 1.00 & Inf \\
\hline$\{1,2,4,5\}$ & .944 & 4.83 & 1.00 & 13.9 & 1.00 & 10.1 & 1.00 & Inf & 1.00 & Inf \\
\hline
\end{tabular}

$C P_{j \cdot \widehat{M}}$ and $L_{j \cdot \widehat{M}}, j=1$, 2, 3, 4 and 5, of post-selection confidence intervals based on Model IV and $\boldsymbol{X}_{E c}$. 


\begin{tabular}{|c|c|c|c|c|c|c|c|c|c|c|}
\hline & \multicolumn{10}{|c|}{ Post-selection Confidence Intervals } \\
\hline & \multicolumn{2}{|c|}{ Naïve } & \multicolumn{2}{|c|}{ Scheffé } & \multicolumn{2}{|c|}{ PoSI } & \multicolumn{2}{|c|}{ EPoSI1 } & \multicolumn{2}{|c|}{ EPoSI2 } \\
\hline $\begin{array}{l}\text { Top Selected } \\
\text { Submodels }\end{array}$ & $\mathrm{CP}_{1 \cdot \widehat{M}}$ & $\mathrm{~L}_{1 \cdot \widehat{M}}$ & $\mathrm{CP}_{1 \cdot \widehat{M}}$ & $\mathrm{~L}_{1 \cdot \widehat{M}}$ & $\mathrm{CP}_{1 \cdot \widehat{M}}$ & $\mathrm{~L}_{1 \cdot \widehat{M}}$ & $\mathrm{CP}_{1 \cdot \widehat{M}}$ & $\mathrm{~L}_{1 \cdot \widehat{M}}$ & $\mathrm{CP}_{1 \cdot \widehat{M}}$ & $\mathrm{~L}_{1 \cdot \widehat{M}}$ \\
\hline$\{1,2,3,4,5\}$ & .976 & 6.05 & 1.00 & 13.3 & 1.00 & 10.4 & .971 & 6.64 & .958 & 9.03 \\
\hline$\{1,2,3,4,5,9\}$ & .979 & 6.06 & 1.00 & 13.3 & 1.00 & 10.4 & .974 & 7.20 & .957 & 10.6 \\
\hline$\{1,2,3,4,5,6\}$ & .988 & 6.07 & 1.00 & 13.3 & 1.00 & 10.4 & .988 & 6.10 & .966 & 12.5 \\
\hline $\begin{array}{l}\text { Top Selected } \\
\text { Submodels }\end{array}$ & $\mathrm{CP}_{2 \cdot \widehat{M}}$ & $\mathrm{~L}_{2 \cdot \widehat{M}}$ & $\mathrm{CP}_{2 \cdot \widehat{M}}$ & $\mathrm{~L}_{2 \cdot \widehat{M}}$ & $\mathrm{CP}_{2 \cdot \widehat{M}}$ & $\mathrm{~L}_{2 \cdot \widehat{M}}$ & $\mathrm{CP}_{2 \cdot \widehat{M}}$ & $\mathrm{~L}_{2 \cdot \widehat{M}}$ & $\mathrm{CP}_{2 \cdot \widehat{M}}$ & $\mathrm{~L}_{2 \cdot \widehat{M}}$ \\
\hline$\{1,2,3,4,5\}$ & .976 & 5.76 & 1.00 & 12.6 & 1.00 & 9.90 & .975 & 5.87 & .964 & 10.0 \\
\hline$\{1,2,3,4,5,9\}$ & .966 & 5.78 & 1.00 & 12.7 & 1.00 & 9.93 & .963 & 7.12 & .949 & 11.8 \\
\hline$\{1,2,3,4,5,6\}$ & .982 & 6.02 & 1.00 & 13.2 & 1.00 & 10.3 & .982 & 6.00 & .974 & 21.8 \\
\hline $\begin{array}{c}\text { Top Selected } \\
\text { Submodels }\end{array}$ & $\mathrm{CP}_{3 \cdot \widehat{M}}$ & $\mathrm{~L}_{3 \cdot \widehat{M}}$ & $\mathrm{CP}_{3 \cdot \widehat{M}}$ & $\mathrm{~L}_{3 \cdot \widehat{M}}$ & $\mathrm{CP}_{3 \cdot \widehat{M}}$ & $\mathrm{~L}_{3 \cdot \widehat{M}}$ & $\mathrm{CP}_{3 \cdot \widehat{M}}$ & $\mathrm{~L}_{3 \cdot \widehat{\boldsymbol{M}}}$ & $\mathrm{CP}_{3 \cdot \widehat{M}}$ & $\mathrm{~L}_{3 \cdot \widehat{M}}$ \\
\hline$\{1,2,3,4,5\}$ & .976 & 6.14 & 1.00 & 13.5 & .998 & 10.6 & .976 & 6.21 & .958 & 9.27 \\
\hline$\{1,2,3,4,5,9\}$ & .984 & 6.45 & 1.00 & 14.1 & 1.00 & 11.1 & .984 & 6.44 & .956 & 14.9 \\
\hline$\{1,2,3,4,5,6\}$ & .991 & 6.20 & 1.00 & 13.6 & 1.00 & 10.6 & .991 & 6.18 & .975 & 13.5 \\
\hline $\begin{array}{l}\text { Top Selected } \\
\text { Submodels }\end{array}$ & $\mathrm{CP}_{4 \cdot \widehat{M}}$ & $\mathrm{~L}_{4 \cdot \widehat{M}}$ & $\mathrm{CP}_{4 \cdot \widehat{M}}$ & $\mathrm{~L}_{4 \cdot \widehat{M}}$ & $\mathrm{CP}_{4 \cdot \widehat{M}}$ & $\mathrm{~L}_{4 \cdot \widehat{M}}$ & $\mathrm{CP}_{4 \cdot \widehat{M}}$ & $\mathrm{~L}_{4 \cdot \widehat{M}}$ & $\mathrm{CP}_{4 \cdot \widehat{M}}$ & $\mathrm{~L}_{4 \cdot \widehat{M}}$ \\
\hline$\{1,2,3,4,5\}$ & .963 & 6.48 & 1.00 & 14.2 & 1.00 & 11.1 & .954 & 7.64 & .952 & 9.67 \\
\hline$\{1,2,3,4,5,9\}$ & .966 & 6.58 & 1.00 & 14.4 & 1.00 & 11.3 & .962 & 8.48 & .959 & 12.7 \\
\hline$\{1,2,3,4,5,6\}$ & .965 & 5.57 & 1.00 & 14.4 & 1.00 & 11.3 & .965 & 6.55 & .963 & 17.3 \\
\hline $\begin{array}{c}\text { Top Selected } \\
\text { Submodels }\end{array}$ & $\mathrm{CP}_{5 \cdot \widehat{M}}$ & $\mathrm{~L}_{5 \cdot \widehat{M}}$ & $\mathrm{CP}_{5 \cdot \widehat{M}}$ & $\mathrm{~L}_{5 \cdot \widehat{M}}$ & $\mathrm{CP}_{5 \cdot \widehat{M}}$ & $\mathrm{~L}_{5 \cdot \widehat{M}}$ & $\mathrm{CP}_{5 \cdot \widehat{M}}$ & $\mathrm{~L}_{5 \cdot \widehat{M}}$ & $\mathrm{CP}_{5 \cdot \widehat{M}}$ & $\mathrm{~L}_{5 \cdot \widehat{M}}$ \\
\hline$\{1,2,3,4,5\}$ & .979 & 5.93 & 1.00 & 13.0 & 1.00 & 10.2 & .978 & 6.25 & .962 & 9.65 \\
\hline$\{1,2,3,4,5,9\}$ & .992 & 6.57 & 1.00 & 14.4 & 1.00 & 11.3 & .990 & 15.0 & .973 & 19.3 \\
\hline$\{1,2,3,4,5,6\}$ & 1.00 & 13.3 & 1.00 & 29.2 & 1.00 & 22.9 & 1.00 & 15.8 & .996 & 84.8 \\
\hline
\end{tabular}

$C P_{j \cdot \widehat{M}}$ and $L_{j \cdot \widehat{M}}, j=1,2,3,4$ and 5, of post-selection confidence intervals based on Model IV and $\boldsymbol{X}_{R l}$. 


\section{References}

Breheny, P. \& Huang, J. (2011), 'Coordinate descent algorithms for nonconvex penalized regression, with applications to biological feature selection', Annals of Applied Statistics 5(1), 232-253.

Fan, J. \& Li, R. (2001), 'Variable selection via nonconcave penalized likelihood and its oracle properties', Journal of the American Statistical Association 96(456), 1348-1360.

Friedman, J., Hastie, T. \& Tibshirani, R. (2010), 'Regularization paths for generalized linear models via coordinate descent', Journal of Statistical Software 33(1), 1-22.

URL: http://www.jstatsoft.org/v33/i01/

Hurvich, C. M. \& Tsai, C.-L. (1990), 'The impact of model selection on inference in linear regression', The American Statistician 44(3), 214-271.

Kabaila, P. (1995), 'The effect of model selection on confidence regions and prediction regions', Econometric Theory 11(3), 537-549.

Kabaila, P. (2005), 'On the coverage probability of confidence intervals in regression after variable selection', Australian and New Zealand Journal of Statistics 47(4), 549-562.

Kabaila, P. \& Giri, K. (2009), 'Upper bounds on the minimum coverage probability of confidence intervals in regression after model selection', Australian and New Zealand Journal of Statistics 51(3), 271-287.

Kabaila, P. \& Leeb, H. (2006), 'On the larger-sample minimal coverage probability of confidence intervals after model selection', Journal of the American Statistical Association 101(474), 619-629.

Koopmans, T. C. (1949), 'Identification problems in economic model construction', Econometrica 17(2), 125-144.

Leeb, H. \& Pötscher, B. M. (2003), 'The finite-sample distribution of postmodel-selection estimators and uniform versus nonuniform approximations', Econometric Theory 19(1), 100-142.

Leeb, H. \& Pötscher, B. M. (2005), 'Model selection and inference: Facts and fiction', Econometric Theory 21(1), 21-59.

Leeb, H. \& Pötscher, B. M. (2006), 'Can one estimate the conditional dis- 
tribution of post-model-selection estimators?', The Annual of Statistics 34(5), 2554-2591.

Leeb, H. \& Pötscher, B. M. (2008), 'Can one estimate the unconditional distribution of post-model-selection estimators?', Econometric Theory 24(2), 338376.

Mallows, C. L. (1973), 'Some comments on $C_{p}$ ', Technometrics 15(4), 661-675.

Pötscher, B. M. (1991), 'Effects of model selection on inference', Econometric Theory $7(2)$, 163-185.

Pötscher, B. M. \& NovÁk, A. J. (1998), 'The distribution of estimators after model selection: large and small sample results', Journal of Statistical Computation and Simulation 60(1), 19-56.

Regal, R. R. \& Hook, E. B. (1991), 'The effects of model selection on confidence interval for the size of a closed population', Statistics in Medicine 10(5), 717721.

Shibata, R. (1984), 'Approximate efficiency of a selection procedure for the number of regression variables', Biometrika 71, 43-49.

Tibshirani, R. (1996), 'Regression shrinkage and selection with the lasso', Journal of the Royal Statistical Society: Series B (Statistical Methodology) 58, 267288.

Zhang, P. (1992), 'Inference after variable selection in linear regression models', Biometrika 79(4), 741-746.

Zou, H. \& Hastie, T. (2005), 'Regularization and variable selection via the elastic net', Journal of the Royal Statistical Society: Series B (Statistical Methodology) 67(2), 301-320. 